\title{
Raumzeitliche Dynamik optisch angeregter Elektron-Loch-Plasmen in Galliumarsenid
}

\author{
Dissertation \\ zur Erlangung des Doktorgrades \\ der Mathematisch-Naturwissenschaftlichen Fakultäten \\ der Georg-August-Universität zu Göttingen
}

vorgelegt von

Ralf Ziebold

aus Neustrelitz

Göttingen, September 2000 
D7

Referent:

Prof. Dr. R. G. Ulbrich

Korreferent:

Prof. Dr. W. Felsch

Tag der mündlichen Prüfung:

25.10.2000 


\section{Inhaltsverzeichnis}

\begin{tabular}{lll}
\hline 1 & Einleitung & 1
\end{tabular}

2 Theoretische Grundlagen und Motivation $\quad 5$

$2.1 \quad$ Bandstruktur und optische Eigenschaften von GaAs . . . . . . . . . . . . . 6

2.2 Ladungsträgerrelaxation in GaAs . . . . . . . . . . . . . . . . . . . . . . 10

2.3 Elektron-Loch-Plasma $\ldots \ldots \ldots \ldots$. . . . . . . . . . . . . . . . . . . . . . . . . 14

2.3 .1 Begriff des Elektron-Loch-Plasmas . . . . . . . . . . . . . . . . . 14

2.3 .2 Zeitentwicklung von Elektron-Loch-Plasmen . . . . . . . . . . . . . 24

\begin{tabular}{lll}
\hline 3 & Flugzeitmeßmethode & 31
\end{tabular}

$3.1 \quad$ Anregungs- und Testexperimente . . . . . . . . . . . . . . . . . . . . . . 32

3.1 .1 Differentielle Transmission $\Delta T$ und Reflexion $\Delta R$. . . . . . . . . 34

$3.2 \Delta R$ und $\Delta T$ in cis und trans Geometrie . . . . . . . . . . . . . . . . . . . . 39

$3.3 \quad$ Erhöhung der Ortsauflösung durch Testen des soh-c Überganges . . . . . . 44

\begin{tabular}{|lll}
4 & Experimentelle Realisierung & 49
\end{tabular}

4.1 Das Lasersystem . . . . . . . . . . . . . . . . . . . . . . . . . . 50

4.1 .1 Die spektrale Filterung . . . . . . . . . . . . . . . . . . . 52

$4.1 .2 \quad$ Charakterisierung der Pulse . . . . . . . . . . . . . . . . . 55

4.2 Die Probenpräparation . . . . . . . . . . . . . . . . . . . . . . . . . . . . . . . . . . . . 57

4.3 Der Meßaufbaul . . . . . . . . . . . . . . . . . . . . . . . . . . . . . 59

$4.4 \quad$ Erste experimentelle Ergebnisse $\ldots \ldots \ldots$. . . . . . . . . . . . . . . . . 63

$4.4 .1 \quad$ Abhängigkeit des $\Delta R$ Signals von der energetischen

Relaxation der Ladungsträger . . . . . . . . . . . . . . . . 63

$4.4 .2 \quad$ Vergleich von $\Delta R(\tau)$ und $\Delta T(\tau)$ in cis und trans Geometrie . . . . 65

$4.4 .3 \quad$ Differentielle Reflexion am soh-c Übergang . . . . . . . . . . . . . . 69

$\begin{array}{lll}5 & \text { EHP-Expansion bei } T_{L}=300 \mathrm{~K} & \mathbf{7 3}\end{array}$

5.1 Experimentelle Untersuchungen . . . . . . . . . . . . . . . . . . . . . . . . . . . . 74

$5.1 .1 \quad$ Erste Hinweise einer EHP-Expansion . . . . . . . . . . . . . . . . . 74

$5.1 .2 \quad$ Flugzeitmessung: Variation der Probendicke . . . . . . . . . . . . . 77

$5.1 .3 \quad$ Flugzeitmessung: Variation der Pumpphotonenenergie . . . . . . . 81

$5.1 .4 \quad$ Flugzeitmessung: Variation der Pumpfluenz . . . . . . . . . . . . . 87 
5.2 Theoretische Beschreibung des Plasmatransportes . . . . . . . . . . . . . . 93

$5.2 .1 \quad$ Hydrodynamisches Diffusionsmodell . . . . . . . . . . . . . . . . . . 94

5.2 .2 Numerische Simulation . . . . . . . . . . . . . . . . . . . . . . 105

5.3 Vergleich Experiment - Simulation . . . . . . . . . . . . . . . 107

5.3.1 Vergleich des Verlaufs von $\Delta R_{\text {trans }}(\tau)$ mit Simulationsrechnungen mit $\mathrm{D}=\mathrm{D}(\mathrm{n}(\mathrm{z}))$ und $\mathrm{D}=$ konst. . . . . . . . . . . . . . . . 108

5.3 .2 Vergleich Experiment - Simulation: Variation der Probendicke . . . 110

$5.3 .3 \quad$ Vergleich Experiment - Simulation: Variation der Pumpfluenz . . . 119

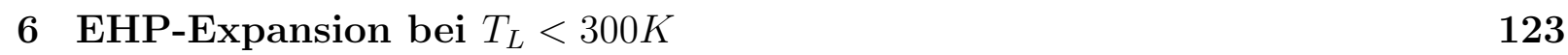

6.1 Experimentelle Untersuchungen . . . . . . . . . . . . . . . . . . . . . . . . . . . . . . 124

6.1 .1 Variation der Gittertemperatur . . . . . . . . . . . . . . . . . 124

$6.1 .2 \quad T_{L}=7 \mathrm{~K}$ : Variation der Probendicke. . . . . . . . . . . . . . . 126

$6.1 .3 \quad T_{L}=4 \mathrm{~K}$ : Variation der Pumpfluenz . . . . . . . . . . . . 126

6.2 Theoretische Beschreibung des Plasmatransportes für $T_{L}<300 \mathrm{~K}$. . . . . . 132

6.3 Vergleich Experiment - Simulation . . . . . . . . . . . . . . . . 138

6.3.1 Vergleich Experiment - Simulation: Variation der Gittertemperatur 138

6.3.2 Vergleich Experiment - Simulation: Variation der Probendicke . . . 139

6.3 .3 Vergleich Experiment - Simulation: Variation der Fluenz . . . . . . 141

\begin{tabular}{lll}
\hline 7 & Diskussion & 151
\end{tabular}

\begin{tabular}{lll}
\hline 8 & Zusammenfassung & 161
\end{tabular}

\begin{tabular}{ll|}
\hline Anhang: Bestimmung des dichteabhängigen Diffusionskoeffizienten & 165
\end{tabular}

Verzeichnis häufig verwendeter Abkürzungen und Symbole 169

\begin{tabular}{ll}
\hline Literaturverzeichnis & 173
\end{tabular} 


\section{Kapitel 1}

\section{Einleitung}

Seit der Erfindung des Transistors durch Bardeen, Brattain und Shockley im Jahre 1949 Sho49 hat die Entwicklung der Halbleitertechnologie die Industriegesellschaft in grundlegendem Maße verändert. Die heutige Entwicklung in der Halbleitertechnologie ist durch eine fortschreitende Miniaturisierung, die bisher ungefähr alle 18 Monate zu einer Verdopplung der Bauelementezahl auf einem Chip führte, und eine Erhöhung der Schaltfrequenzen gekennzeichnet. Voraussetzung für diese rasante technologische Entwicklung ist ein immer besseres Verständnis der involvierten physikalischen Prozesse auf immer kleineren Orts- und Zeitskalen. Dementsprechend wurden in den letzten Jahrzehnten neue Untersuchungsmethoden mit dem Ziel entwickelt, die grundlegenden physikalischen Prozesse in den unterschiedlichen Halbleitermaterialien zu verstehen und gegebenenfalls Impulse für neue Produkte zu geben.

Die Entwicklung von Lasersystemen, welche ultrakurze Pulse mit kürzesten Pulsdauern von $\sim 6$ fs erzeugen, hat es in den letzten Jahren ermöglicht, die in Halbleitern ablaufenden Prozesse direkt in der Zeitdomäne mit optischen Methoden zu spektroskopieren [Die96]. So sind die Abläufe nach einer räumlich homogenen Anregung von Halbleitermaterialien mit ultrakurzen optischen Pulsen weitestgehend verstanden [Sha99]. Erst in jüngerer Vergangenheit hat man versucht, die hohe zeitliche Auflösung der fs-Spektroskopie mit der hohen räumlichen Auflösung von mikroskopischen Techniken zu verknüpfen [Koc00]. So wurden für die zeitaufgelöste Untersuchung des lateralen Transportes (parallel zur Probenoberfläche) optisch angeregter Ladungsträger Methoden der optischen Nahfeldmikroskopie [Gro98, Ent00, der konfokalen Mikroskopie [Vol99] und auch Maskentechniken [Het00] eingesetzt. In dieser Arbeit wird nun eine Methode vorgestellt, die die Untersuchung des Ladungsträgertransportes nicht parallel sondern senkrecht zur Probenoberfläche mit einer sub- $\mu$ m räumlichen und sub-ps zeitlichen Auflösung ermöglicht.

Als eine erste Anwendung wird mit dieser Flugzeitmeßmethode die raumzeitliche Dynamik von optisch mit einer hohen Dichte $n>10^{18} \mathrm{~cm}^{-3}$ angeregten Elektronen und Löchern in dem direkten Halbleiter Galliumarsenid (GaAs) untersucht. Bei der Anregung einer hohen Dichte von Elektronen und Löchern sind deren Eigenschaften aufgrund unter- 
schiedlicher Wechselwirkungsmechanismen von ihrer Dichte abhängig. Für dieses System stark miteinander wechselwirkender Ladungsträger wird in der Literatur (siehe z.B. Pey93) der Begriff des Elektron-Loch-Plasmas (EHP ${ }^{1}$ ) eingeführt. Mit der Verfügbarkeit von intensiven optischen Laserpulsen wurden die Eigenschaften von Elektron-LochPlasmen weitestgehend untersucht (siehe z.B. Pey93, Kli95]). Für die Interpretation ihrer Meßergebnisse wird von einigen Autoren eine schnelle Ausbreitung (Expansion) der oberflächennah mit einer Absorptionslänge $1 / \alpha<1 \mu \mathrm{m}$ angeregten Elektron-LochPlasmen in die Tiefe der Probe diskutiert (siehe z.B. [Tse89, Nak92, Pol96, Gus97]). Die Frage nach der Ursache einer schnellen Plasmaexpansion senkrecht zur Oberfläche ist Gegenstand kontroverser Diskussionen gewesen und kann bis heute nicht zufriedenstellend beantwortet werden (siehe z.B. [Pil85]). Ziel der hier vorliegenden Arbeit ist es nun, mit Hilfe der von uns entwickelten Flugzeitmeßmethode die Elektron-Loch-PlasmaExpansion in GaAs, insbesondere für frühe Zeiten $<100 \mathrm{ps}$, zu untersuchen, um daraus Rückschlüsse auf die der Expansion zugrundeliegenden treibenden Kraft ziehen zu können.

Diese Arbeit ist in 8 Kapitel untergliedert. Nach dieser Einleitung werden im 2. Kapitel zum einen die linear optischen Eigenschaften des untersuchten Halbleiters GaAs vorgestellt, zum anderen werden die wesentlichen Relaxationsmechanismen diskutiert, die nach der Anregung von GaAs mit einem kurzen optischen Puls ablaufen. Im letzten Abschnitt des 2. Kapitels werden die grunglegenden Eigenschaften von Elektron-Loch-Plasmen und die in der Literatur diskutierten möglichen Ursachen einer Elektron-Loch-Plasma-Expansion erläutert. Nach der Darlegung der theoretischen Grundlagen und der Motivation wird im 3. Kapitel das Konzept der Flugzeitmeßmethode und anschließend im 4. Kapitel die konkrete experimentelle Realisierung vorgestellt. Die experimentellen Ergebnisse der Flugzeituntersuchungen zur Elektron-Loch-Plasma-Expansion in GaAs werden in den Kapiteln 5 für die Messungen bei Raumtemperatur und 6 für die Messungen bei tiefen Gittertemperaturen $T_{L}<300 \mathrm{~K}$ vorgestellt. Um aus den Meßergebnissen Rückschlüsse auf die treibende Kraft der Elektron-Loch-Plasma-Expansion ziehen zu können, werden die Meßergebnisse mit Simulationen im Rahmen eines einfachen hydrodynamischen Diffusionsmodells verglichen. Dieser Vergleich legt den Fermidruck als die wesentliche Ursache der untersuchten Elektron-Loch-Plasma-Expansion nahe. Eine Diskussion anderer die Plasmaexpansion beeinflussender Faktoren erfolgt in Kapitel 7.

\footnotetext{
${ }^{1}$ EHP: engl. electron-hole plasma
} 


\section{Kapitel 2}

\section{Theoretische Grundlagen und Motivation}

In diesem Kapitel werden die wesentlichen theoretischen Grundlagen und die Motivation für die in der Arbeit durchgeführten Experimente dargelegt. Der erste Abschnitt beinhaltet die linear optischen Eigenschaften des untersuchten Halbleiters GaAs. Danach werden die Relaxationsvorgänge nach Anregung von GaAs mit einem kurzen $(\sim f s)$ optischen Lichtpuls vorgestellt, um im letzten Abschnitt auf die Anregung, die wesentlichen Eigenschaften und insbesondere die schnelle Ausbreitung von Elektron-Loch-Plasmen in Halbleitern einzugehen.

\subsection{Bandstruktur und optische Eigenschaften von GaAs}

Die optischen Eigenschaften von Halbleitern im sichtbaren und nahen infraroten Bereich werden durch ihre elektronische Bandstruktur (siehe Abb. 2.1) bestimmt. Der hier untersuchte binäre Halbleiter GaAs kristallisiert in der Zinkblendestruktur und besitzt eine direkte Bandlücke am $\Gamma$-Punkt von $E_{G}=1,428 \mathrm{eV}$ bei $T=300 \mathrm{~K}$ bzw. $E_{G}=1,519 \mathrm{eV}$ bei $T=2 \mathrm{~K}$ [Lan87]. Im folgenden werden im Bereich des $\Gamma$-Punktes neben dem untersten Leitungsband (c) die drei obersten Valenzbänder betrachtet. Die oberen zwei bei $k=0$ entarteten Valenzbänder sind das schwere Lochband (heavy hole band hh) und das leichte Lochband (light hole band lh). Das durch die Spin-Bahn-Wechselwirkung abgespaltene Valenzband (split off hole band soh) besitzt am $\Gamma$-Punkt eine um $\Delta_{\text {soh }}=0,341 \mathrm{eV}(T=0 \mathrm{~K})$ geringere Energie [Lan87]. Diese drei Valenzbänder und das unterste Leitungsband sind jeweils zweifach spinentartet. Das Leitungsband (c) besitzt Seitentäler an den $L$ - und $X$ Punkten, die einen energetischen Abstand vom Minimum am $\Gamma$-Punkt von $\Delta_{\Gamma-L}=0,27 \mathrm{eV}$ bzw. $\Delta_{\Gamma-X}=0,48$ bei $T=300 \mathrm{~K}$ [Lan87] besitzen.

In der Nähe der Bandextrema kann man die Bänder parabolisch in $k$ approximieren. 


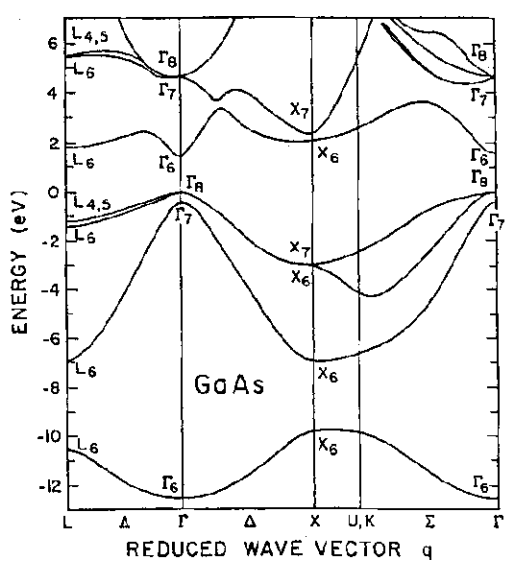

Abbildung 2.1: Bandstruktur von GaAs $(T=300 \mathrm{~K})$ nach Che87.

In der isotropen Effektive-Masse-Näherung ergibt sich:

$$
E(\vec{k})=E(k)=\frac{\hbar^{2} k^{2}}{2 m^{*}} .
$$

Hierbei bezeichnet $\vec{k}$ den Wellenvektor des Elektrons bzw. des Loches und $m^{*}$ die effektive Masse des jeweiligen Bandes, wobei $\left(1 / m^{*}\right)$ i. Allg. Tensorcharakter besitzt. Da Anisotropieeffekte in dieser Arbeit keine Rolle spielen, wird in dieser Arbeit der Tensorcharakter der effektiven Masse vernachlässigt.

Für die in dieser Arbeit durchgeführten Experimente bei unterschiedlichen Gittertemperaturen $T_{L}$ ist die Temperaturabhängigkeit der Bandlücke $E_{G}\left(T_{L}\right)$ zu beachten. Diese wird von Panish und Casey [Pan69] in einem analytischen Ausdruck für GaAs angegeben mit (siehe auch Abb. 2.2):

$$
E_{G}(T)=1,522 \mathrm{eV}-5,8 \times 10^{-4} \mathrm{eV} / \mathrm{K} \cdot T^{2} /(T+300 \mathrm{~K}) \mathrm{eV} ; \quad(T \text { in } \mathrm{K}) .
$$

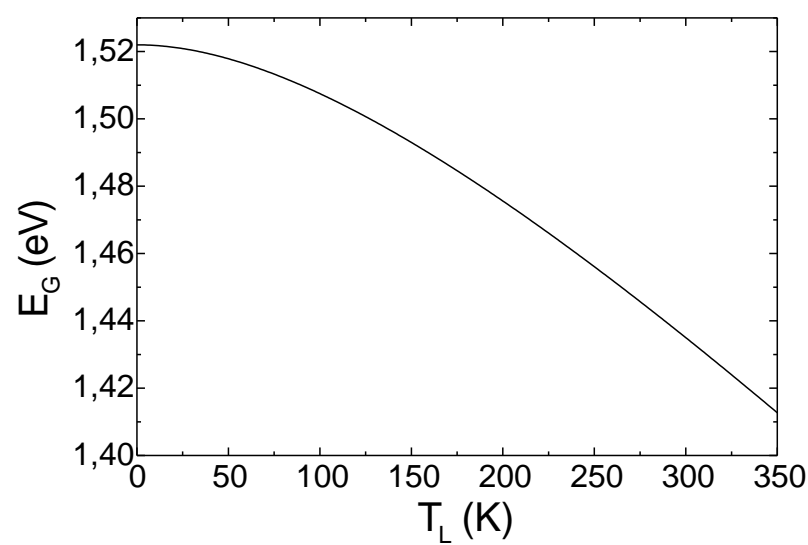

Abbildung 2.2: Abhängigkeit der Bandlücke $E_{G}$ von der Temperatur $T_{L}$ 
Bei der Absorption eines Photons der Energie $\hbar \omega>E_{G}$ wird ein Elektron aus einem Valenzband in das Leitungsband angeregt. Im folgenden wird das so angeregte Leitungsbandelektron als Elektron und die gleichzeitig generierte Vakanz im Valenzband als Loch bezeichnet (siehe auch Pey93]). Das Absorptionsvermögen oberhalb der Bandlücke ergibt sich aus der Summe aller Beiträge der bei der eingestrahlten Photonenenergie $\hbar \omega$ möglichen Übergänge, bei denen Elektronen aus einem Valenzband in ein Leitungsband angeregt werden können (vgl. Gl. (2.5)). Da im sichtbaren Spektralbereich der Photonenimpuls klein gegenüber der Ausdehnung der ersten Brillouinzone ist, erfolgen die optischen Übergänge senkrecht im k-Raum. Der frequenzabhängige Absorptionskoeffizient $\alpha(\omega)$ läßt sich wie folgt bestimmen [Woo72]:

$$
\begin{array}{cll} 
& \alpha(\omega)= & \frac{e^{2} \pi}{m_{0}^{2} c \omega n \epsilon_{0}} \cdot|\vec{e} \cdot \vec{P}|^{2} \cdot \sum_{\nu} D_{v}(\hbar \omega) \\
\text { mit } \quad & \quad \ldots & \text { Elementarladung } \\
\epsilon_{0} \ldots & \text { Dielektrizitätskonstante des Vakuums } \\
c \ldots & \text { Lichtgeschwindigkeit } \\
n \ldots & \text { Brechungsindex } \\
& \vec{e} \ldots & \text { Polarisationsvektor des Lichtes }
\end{array}
$$

$\vec{P}$ ist hierbei das Übergangsmatrixelement vom Zustand $\mid i>$ in den Zustand $\mid j>$ mit

$$
\vec{P}=\langle i|\vec{p}| j\rangle
$$

und dem Impulsoperator $\vec{p}$. Die kombinierte Zustandsdichte $D_{v}$ für den Übergang aus jeweils einem Valenzband ( $v:$ hh, lh, soh) ins Leitungsband (c) ergibt sich aus (BZ: Brillouinzone):

$$
D_{v}(\hbar \omega)=\frac{2}{(2 \pi)^{3}} \int_{\mathrm{BZ}} \mathrm{d}^{3} \mathrm{k} \delta\left(\mathrm{E}_{\mathrm{c}}(\overrightarrow{\mathrm{k}})-\mathrm{E}_{\mathrm{v}}(\overrightarrow{\mathrm{k}})-\hbar \omega\right)
$$

Die Berechnung der kombinierten Zustandsdichte [Böh92] in diesem Modell bei $\mathrm{T}=300 \mathrm{~K}$ zeigt, daß bei einer Anregung des GaAs-Kristalls mit einer Photonenenergie von $2 \mathrm{eV}$, wie sie z.T. auch im Experiment verwendet wird, ca. $60 \%$ der Übergänge pro Zeiteinheit aus dem hh-, ca. 30\% aus dem lh- und ca. 10\% aus dem soh-Band erfolgen.

Im allgemeinen reicht dieses einfache Modell zur Beschreibung der Absorption in der Nähe der Bandkante $E_{G}$ nicht aus, da hier die Coulombwechselwirkung zwischen dem Elektron und Loch vernachlässigt wird. Eine Berücksichtigung dieser Wechselwirkung führt zu einer Bildung diskreter Absorptionslinien (Exzitonen) unterhalb der Bandlücke und auch oberhalb von $E_{G}$ zu einer erhöhten Absorption [Ell57] (siehe Abb. 2.3).

In dieser Arbeit richtet sich das Hauptaugenmerk jedoch auf die Dynamik von freien Elektronen und Löchern, die mit Photonenenergien $\hbar \omega>E_{G}$ angeregt werden. 

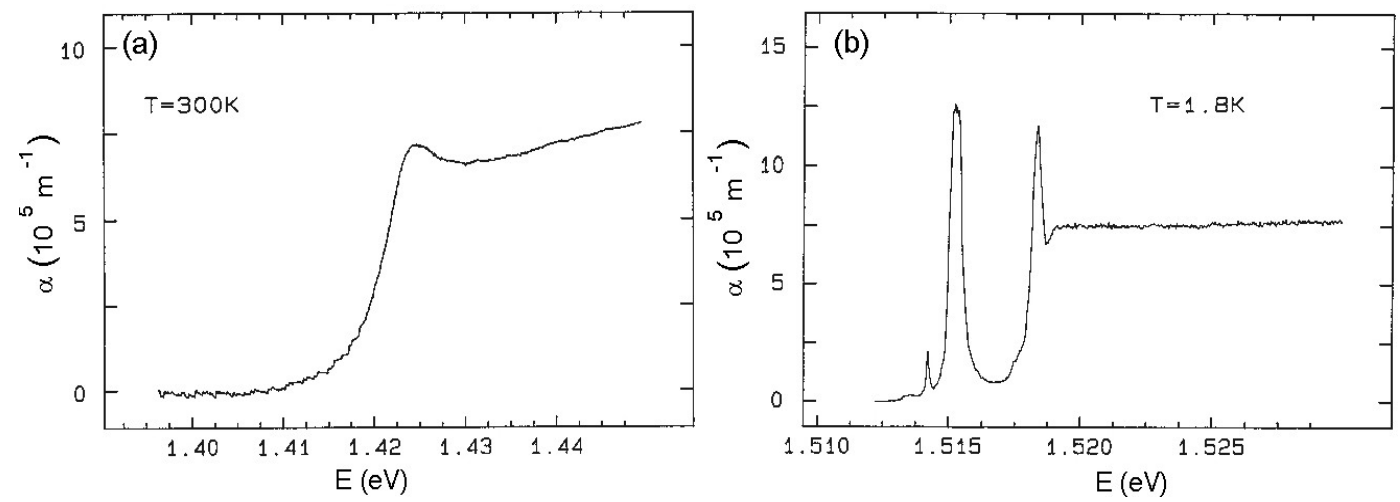

Abbildung 2.3: Absorptionsspektren von GaAs bei (a) $T=300 \mathrm{~K}$ und (b) $T=1,8 \mathrm{~K}$ Tri88

\subsection{Ladungsträgerrelaxation in GaAs}

Nach Anregung eines sich im thermischen Gleichgewicht befindenden Halbleiters mit einem kurzen (Pulslänge: $\tau_{\text {Puls }} \sim 100 \mathrm{fs}$ ) optischen Puls relaxiert dieser über unterschiedliche Relaxationsprozesse wieder in das thermische Gleichgewicht. Die Relaxationsprozesse können prinzipiell in 4 sich teilweise überlappende Regime eingeteilt werden [Sha99] (siehe Abb. 2.4.

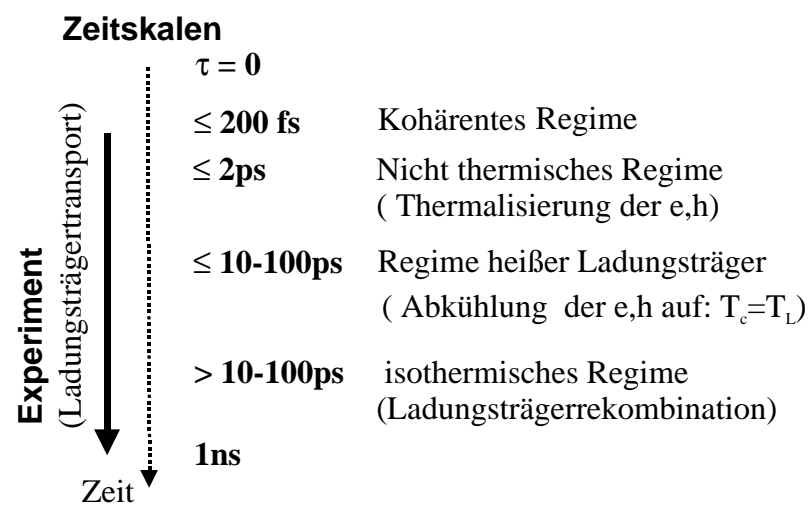

Abbildung 2.4: Relaxationsregime in optisch angeregten Halbleitern mit ihren Zeitskalen (links: im Experiment untersuchte Zeitskala).

(1) Kohärentes Regime: Bei einer optischen Anregung von Halbleitern mit einem kurzen optischen Puls besitzen die Anregungen zunächst eine feste Phasenbeziehung mit dem anregenden elektromagnetischen Feld. Die Schwierigkeit bei der Untersuchung kohärenter Phänomene in Halbleitern besteht darin, daß im Unterschied zur Optik an Atomen in Halbleitern die Phasenkohärenz aufgrund der unterschiedlichen Wechselwirkungen im Halbleiter innerhalb relativ kurzer Zeit $(\sim 100 \mathrm{fs}-1 \mathrm{ps})$ zerstört wird. Die Eigenschaft der Kohärenz führt zu einer Vielzahl interessanter Phänomene wie z.B. dem Photonenecho, welches zuerst von Kurnitt [Kur64] in Rubin gefunden wurde, oder dem 


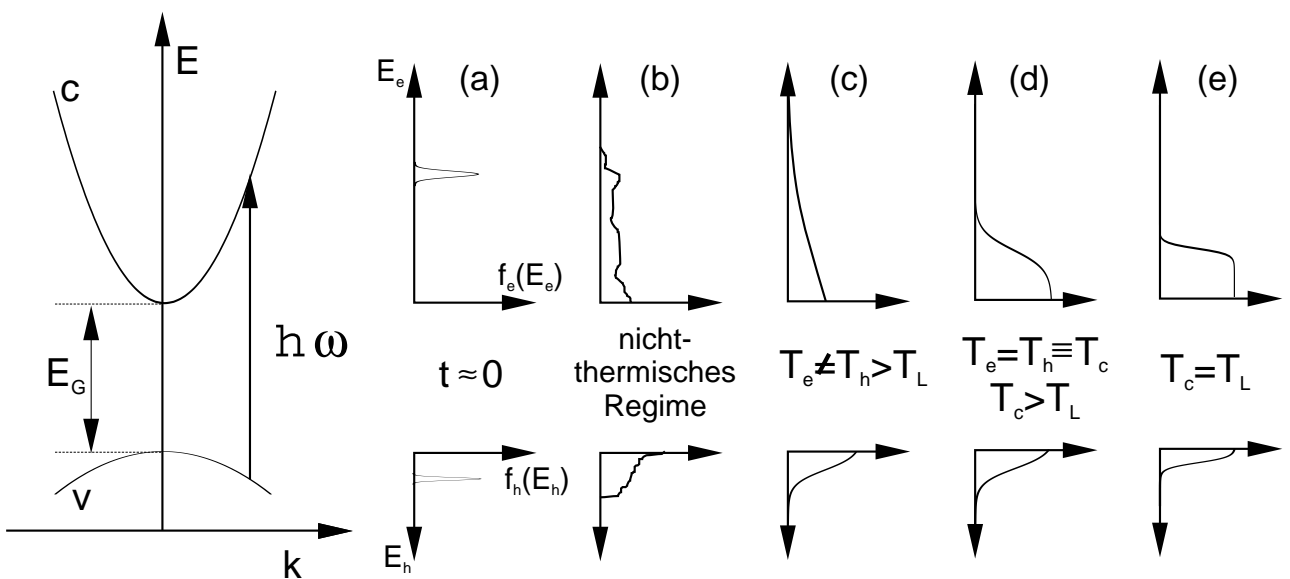

Abbildung 2.5: Schematische Darstellung der optischen Anregung in einem Halbleiter mit einem Valenzband (v) und einem Leitungsband (c). Rechts sind die ursprünglichen Verteilungsfunktionen der Elektronen $f_{e}\left(E_{e}\right)$ und Löcher $f_{h}\left(E_{h}\right)$ und ihre zeitlichen Entwicklungen schematisch dargestellt.

AC-Stark Effekt, welcher in Halbleitern zuerst von Fröhlich [Frö85] bzw. Mysyrowitz Mys86 entdeckt wurde. In der jüngeren Vergangenheit wurden auch Rabioszillationen Sch99] und selbstinduzierte Transmission (SIT) Gie98 in Halbleitern gefunden.

Bei den in dieser Arbeit verwendeten Photonenenergien $\hbar \omega>E_{G}$ werden freie Ladungsträger angeregt, für die in GaAs Dephasierungszeiten von ca. $14-44 \mathrm{fs}$ bei Anregungsdichten von $1,5 \times 10^{17}$ bis $7 \times 10^{18} \mathrm{~cm}^{-3}$ [Bec88] gefunden wurden. Der dominierende, die Kohärenz zerstörende Streuprozeß ist hier die e-e Streuung.

(2) Nichtthermisches Regime: Bei der Anregung eines Halbleiters mit einer Photonenenergie $\hbar \omega>E_{G}$ werden Elektronen und Löcher generiert, deren ursprüngliche Verteilungsfunktionen im k-Raum $f_{c}\left(\vec{k}_{c}\right)>0(\mathrm{c}=\mathrm{e}, \mathrm{h})$ durch die bei der jeweiligen Photonenenergie möglichen optischen Übergänge bestimmt wird. Diese Verteilungsfunktionen $f_{c}\left(\vec{k}_{c}\right)$ können unter Berücksichtigung der Bandstruktur $E(\vec{k})$ auf energetische Verteilungsfunktionen $f_{c}\left(E_{c}\right)$ reduziert werden ${ }^{1}$. Die zeitliche Entwicklung der energetischen Verteilungsfunktionen von Elektronen und Löchern für einen Zweibandhalbleiter sind schematisch in der Abbildung 2.5 dargestellt. Für frühe Zeiten (siehe Abb. 2.5(a,b)) entsprechen die Verteilungsfunktionen nicht thermischen Gleichgewichtsverteilungen. Solche nichtthermischen Ladungsträgerverteilungen wurden in GaAs zum ersten Mal mit Hilfe der spektral aufgelösten differentiellen Transmission von Oudar Ou85] untersucht. Die Entwicklung zu einer thermischen Verteilung erfolgt bei mittleren und hohen Anregungsdichten über die Streuung der Ladungsträger untereinander [Bec88].

\footnotetext{
${ }^{1}$ Informationen über eine mögliche anisotrope Ladungsträgerverteilung im k-Raum gehen dabei jedoch verloren.
} 
Kapitel 2 Theoretische Grundlagen und Motivation

(3) Regime heißer Ladungsträger: Nach Ablauf einer genügend großen Zahl von Streuprozessen entwickeln sich die Verteilungsfunktionen der Elektronen und Löcher jeweils zu thermischen Verteilungen, wobei der energetische Nullpunkt die jeweilige Bandkante ist (siehe Abb. 2.5). Eine Beschreibung dieser Verteilungen mit Hilfe sogenannter Quasitemperaturen $T_{e}, T_{h}$ ist aufgrund der Tatsache gerechtfertigt, daß die Ladungsträgerrekombination ein im Vergleich zu den anderen ablaufenden Prozessen langsamer Prozeß $(\sim \mathrm{ns})$ ist. I. Allg. besitzen Elektronen und Löcher anfänglich unterschiedliche Temperaturen $T_{e} \neq T_{h}$ (siehe Abb. 2.5(c)), die sich aufgrund von e-h Streuprozessen auf $T_{e}=T_{h} \equiv T_{c}$ angleichen (siehe Abb. 2.5(d)). Die Abkühlung der heißen Ladungsträger auf $T_{c}=T_{L}$ erfolgt über die Emission von Phononen.

Bei Elektronen mit hohen Überschußenergien ist die Emission von longitudinal optischen (LO) Phononen über die Fröhlich-Wechselwirkung der dominierende Prozeß für die energetische Relaxation. Die mittlere Elektron-Phonon-Stoßzeit beträgt bei Anregungsdichten bis $10^{17} \mathrm{~cm}^{-3}$ etwa 150fs (siehe z.B. [Lin79]: 120fs, [Fas90]: 165fs). Bei höheren Anregungsdichten wird die LO-Phonon-Emission weniger effektiv [Kas85]. Dieses Verhalten wird mit der Abschirmung der Fröhlich-Wechselwirkung durch das Elektron-LochPlasma Yof81, Kas85, Sar88, Col89, teilweise aber auch mit der Reabsorption heißer Phononen [Poe87, Lug87], erklärt. Ist die kinetische Energie der Elektronen größer als der energetische Abstand des Leitungsbandminimums zu den Nebenminima an den $L$ - bzw. $X$-Punkten $\Delta_{\Gamma-L}$ bzw. $\Delta_{\Gamma-X}$, so ist auch eine Streuung der Elektronen in die Seitentäler (Intervalley Streuung) möglich [Col83, Ulb89, Coc97]. Diese erfolgt über die kurzreichweitige Deformationspotentialstreuung an longitudinal optischen und akustischen Phononen [Zol89].

Wenn die Emission optischer Phononen energetisch nicht möglich ist, ist in GaAs bei niedrigen Anregungsdichten die Emission akustischer Phononen durch die piezoelektrische Wechselwirkung der dominante Energieverlustmechanismus Ulb73. Bei großen Anregungsdichten $\left(n>10^{18} \mathrm{~cm}^{-3}\right)$ überwiegt aufgrund der Abschirmung der piezoelektrischen Wechselwirkung die kurzreichweitige akustische Deformationspotentialwechselwirkung [Pug81].

Die energetische Relaxation der Löcher erfolgt bei hohen Überschußenergien über die Emission von longitudinal und transversal optischen (LO, TO) Phononen und findet auf einer, im Vergleich zu der Relaxation der Elektronen, kürzeren Zeitskala statt (siehe z.B. [Lan96, Sha99]).

Experimentell wurde die Ladungsträgertemperatur als Funktion der Zeit $\tau$ nach Eintreffen des anregenden Laserpulses $T_{c}=T_{c}(\tau)$ mit zeitaufgelösten Meßmethoden wie der zeit- und spektral aufgelösten differentiellen Transmission (siehe Kap. 3.1.1) [Leh79, Lin79] und der zeitaufgelösten Lumineszenz (siehe z.B. [Tan80]) bestimmt. In der Abbildung 2.6 sind die experimentell für GaAs bestimmten Abkühlkurven zusammen mit theoretisch bestimmten Kurven für eine Gittertemperatur von $T_{L}=10 \mathrm{~K}$ dargestellt [Sha99].

Man erkennt, daß sich die Ladungsträger nach 10ps auf $140-60 \mathrm{~K}$ abgekühlt haben. Eine weitere Abkühlung auf $T_{c}<30 K$ geschieht jedoch erst innerhalb einer Verzögerungszeit von $\tau>100$ ps. Die schnelle Abkühlung auf $T_{c} \sim 100 \mathrm{~K}$ ist auf die Emission von optischen Phononen als sehr effizienten Energieverlustmechanismus für Ladungsträger 


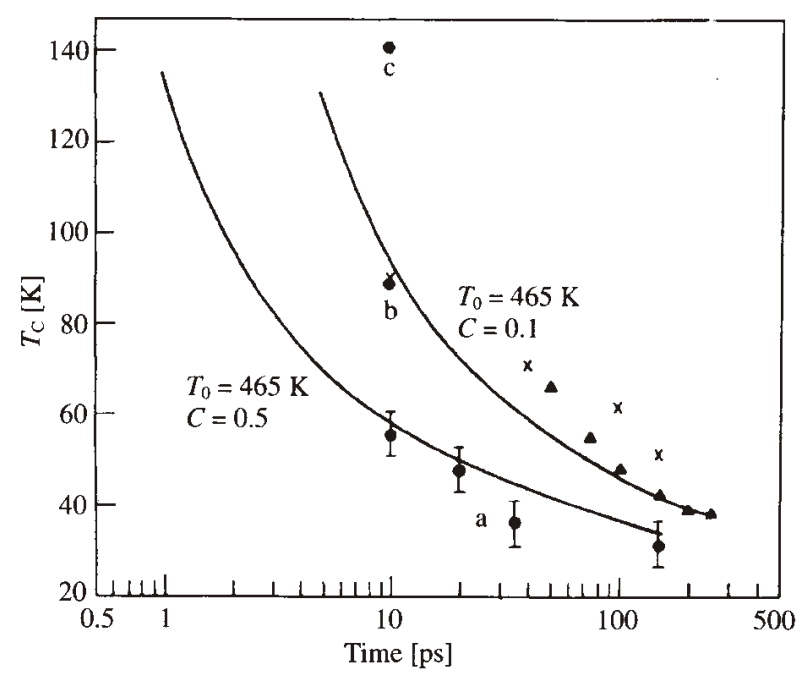

Abbildung 2.6: Abkühlkurven für GaAs $\left(T_{L}=10 K\right)$ : Kreise [Leh79]: $n=($ a) $7 \times$ $10^{16} \mathrm{~cm}^{-3}$, (b) $3 \times 10^{17} \mathrm{~cm}^{-3}$, (c) $1 \times 10^{18} \mathrm{~cm}^{-3}$; Dreiecke: [Lin79]; Kreuze [Tan80; Linie: theoretisch berechnete Kurven .

mit Energien $E_{k i n}>E_{L O-P h o n o n}$ zurückzuführen. Eine weitere Abkühlung erfolgt über den weniger effizienten Mechanismus der Emission von akustischen Phononen (siehe oben).

(4) Isothermisches Regime: Am Ende des Regimes heißer Ladungsträger haben die Ladungsträger die gleiche Temperatur wie das Gitter. Dieser Zustand ist jedoch immer noch ein Nichtgleichgewichtszustand, da die Anzahl der vorhandenen Ladungsträger nicht der Anzahl im thermischen Gleichgewicht entspricht. Über eine strahlende oder nichtstrahlende Rekombination der Ladungsträger gelangt der Halbleiter wieder auf einer $\sim$ ns Zeitskala in das thermische Gleichgewicht.

\subsection{Elektron-Loch-Plasma}

\subsubsection{Begriff des Elektron-Loch-Plasmas}

Im Abschnitt 2.1 wurden die linear optischen Eigenschaften von GaAs vorgestellt. Die bei einer intensiven optischen Anregung mit einem kurzen Laserpuls erzeugte hohe Dichte an Elektronen und Löchern führt nun zu einer signifikanten Änderung der elektronischen Bandstruktur sowie der optischen Eigenschaften.

Eine hohe Elektron-Loch-Teilchendichte führt zum einen aufgrund von Abschirmungseffekten zu einer Veränderung der Streurate der über die Coulombwechselwirkung vermittelten Ladungsträger-Phononen-Streuung (z.B. Ladungsträger-LO-Phonon-Streuung über die Fröhlich-Wechselwirkung), die im letzten Abschnitt schon diskutiert wurde.

Zum anderen führt eine hohe Teilchendichte aufgrund von Austausch- und Korrelations- 


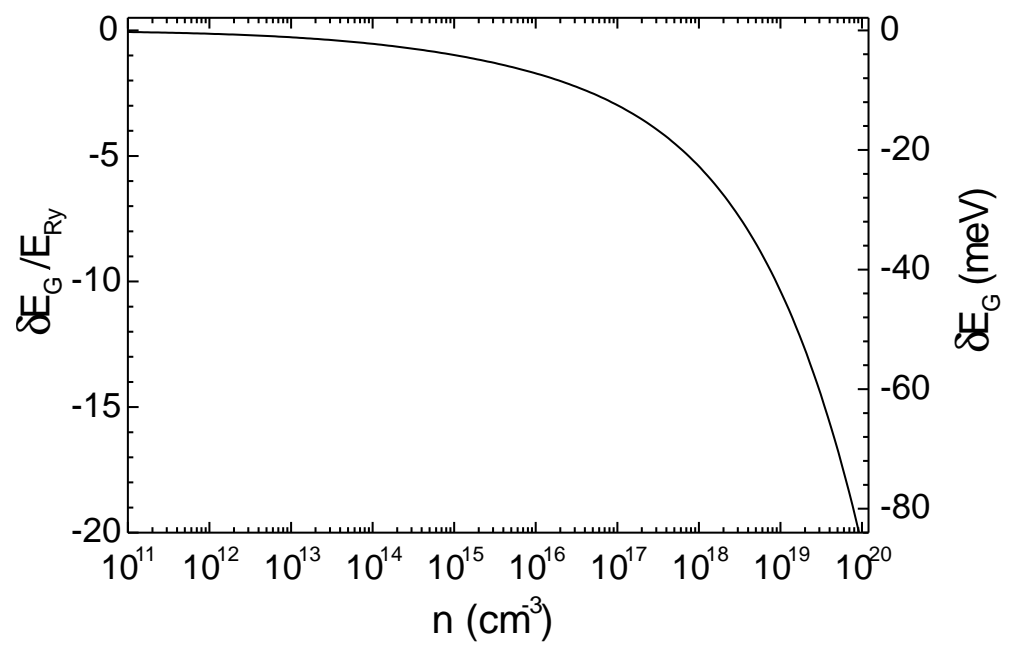

Abbildung 2.7: Bandkantenrenormierung $\delta E_{G}$ als Funktion der Ladungsträgerdichte $n$ für GaAs bei $T=300 \mathrm{~K}$ (GaAs: $E_{R y}=4,2 \mathrm{eV}$ )

effekten auch zu einer Renormalisierung der Eigenenergien [Pey93, Zim87]. In der einfachsten Näherung kann man diese Renormalisierung der Eigenenergien mit einer Änderung der Bandlücke $E_{G}$ in Abhängigkeit der Ladungsträgerdichte $n$ bei ansonsten unveränderter Bandstruktur (Näherung: starre Bänder) berücksichtigen: $E_{G}(n)=E_{G}^{0}+\delta E_{G}(n)$. Ein universeller Ausdruck, der diesen Zusammenhang beschreibt, wurde von Vashishta und Kalia Vas82 abgeleitet:

$$
\frac{\delta E_{G}}{E_{R y}}=E_{x c}+n \frac{\partial E_{x c}}{\partial n}
$$

mit der Summe aus Austausch- und Korrelationsenergie $E_{x c}$ ('xc' steht hier für exchange und correlation):

$$
E_{x c}=\frac{4,8316+5,0879 r_{s}}{0,0152+3,0426 r_{s}+r_{s}^{2}}
$$

und dem dimensionslosen Teilchenabstand $r_{s}$ :

$$
r_{s}=\left[\begin{array}{ll}
\frac{1}{n a_{0}^{3}} & \frac{3}{4 \pi}
\end{array}\right]^{1 / 3} .
$$

Hierbei sind $E_{R y}$ die Bindungsenergie und $a_{0}$ der Bohrradius des 1 s Exzitons. Mit den Werten für GaAs ergibt sich die in Abbildung 2.7 dargestellte Änderung der Bandlücke $\delta E_{G}$ in Abhängigkeit der Ladungsträgerdichte $n$. Man erkennt, daß die Bandlückenenergie mit zunehmender Teilchendichte abnimmt. Mit zunehmender Teilchendichte, beginnend bei einer Niedrigdichte-Anregung, kommt es zu einem Übergang von der isolierenden exzitonischen Phase zu dem metallischen Elektron-Loch-Plasma (EHP) [Ric77, Hen77, dem Mott-Übergang [Mot74]. Während die Energie der Bandlücke $E_{G}$ mit zunehmender 
Dichte abnimmt, bleibt die Energie der Exzitonen $E_{\text {exc }}$ annähernd konstant ${ }^{2}$ [Feh82]. Der Grund dafür ist, daß die Renormalisierung der Bandlücke von der gleichzeitigen Abnahme der Exzitonenbindungsenergie kompensiert wird. Der Mott-Übergang findet bei der Dichte (Mottdichte) $n_{M} \approx 10^{15}-10^{16} \mathrm{~cm}^{-3}$ in GaAs statt, bei der die Energie des Elektron-Loch-Kontinuums die Exzitonenenergie schneidet. In der Abbildung 2.7 entspricht das in etwa der Dichte, bei der $\delta E_{G}(n)=-1 E_{R y}$ ist $t^{3}$

Unabhängig von dieser Definition des Elektron-Loch-Plasmas (EHP) über Grundzustandsenergien im thermischen Gleichgewicht, wird der Begriff des EHP-s in der Literatur (siehe z.B. [Tse89, Pol99]) auch für Elekronen und Löcher verwendet, die als e-h Paar mit einer Photonenenergie $\hbar \omega>E_{G}$ angeregt werden. Bei diesem Gebrauch des Begriffes für Nichtgleichgewichtszustände ist die Frage, ob das angeregte Elektron-Loch-Plasma oder aber die Exzitonen den stabilen Grundzustand des Systems bilden, nicht von Bedeutung. In dieser Form soll auch in dieser Arbeit der Begriff des Elektron-Loch-Plasmas verwendet werden.

In Halbleitern mit einer indirekten Bandlücke wie Silizium und Germanium ist die Existenz einer thermodynamischen Hochdichte-Phase, nämlich der der Elektron-Loch Flüssigkeit (EHL ${ }^{4}$, nachgewiesen ${ }^{5}$, deren Existenz zuerst von Keldysh 1968 für die Interpretation einer energetisch unterhalb der Exzitonenlinie liegende Lumineszenzlinie in Ge vorgeschlagen wurde [Kel68]. Das EHL ist mit einem lokalen Minimum des chemischen Potentials $\mu$ als Funktion der Teilchendichte $n$ verknüpft und entsteht über einen Phasenübergang erster Ordnung aus der exzitonischen Phase. Das EHL ist durch eine wohldefinierte Begrenzungsfläche mit einer Oberflächenspannung und einer Gleichgewichtsdichte charakterisiert JJef83, Pey93. Die Existenz einer EHL-Phase wurde auch für direkte binäre Halbleiter wie GaAs vorhergesagt [Zim87], konnte jedoch experimentell nicht nachgewiesen werden [Hil78, Nak92, Kli95]. Eine Ursache hierfür könnte die im Vergleich zu indirekten Halbleitern schnellere strahlende Rekombination sein, die die Relaxation in die möglicherweise vorhandene Gleichgewichtsphase des EHL-s verhindert Pey93, Kli95.

\footnotetext{
${ }^{2}$ Sowohl $E_{G}$ als auch $E_{e x c}$ sind hierbei Zweiteilchenenergien. Die Exzitonenenergie $E_{\text {exc }}$ kann über die kleinstmögliche Photonenenergie $\hbar \omega$, die für die Erzeugung eines Elektron-Loch-Paares benötigt wird, definiert werden. Die Bandlückenenergie $E_{G}$ ergibt sich aus der Summe der Exzitonenenergie $E_{e x c}$ und der Energie, die benötigt wird, das Elektron-Loch-Paar örtlich auf einen Abstand $d=\infty$ zu trennen (Exzitonbindungsenergie).

${ }^{3}$ Neuere theoretische Untersuchungen mit Hilfe von Quanten-Monte-Carlo-Simulationen [Zhu96] ergeben eine Stabilität der exzitonischen Phase gegenüber dem EHP bis zu $r_{s}=0,5$. Dies entspricht in GaAs einer Dichte von ca. $8 \times 10^{17} \mathrm{~cm}^{-3}$.

${ }^{4}$ EHL: engl. electron hole liquid

${ }^{5}$ Einen Überblick über die vielfältigen Eigenschaften von EHL-s findet man z.B. in [Jef83]
} 


\subsubsection{Zeitentwicklung von Elektron-Loch-Plasmen}

Die zeitliche Entwicklung von optisch generierten Elektron-Loch-Plasmen wird von der im Abschnitt 2.2 diskutierten Ladungsträgerrelaxation, der (nichtlinearen) Rekombination und von der örtlichen Ausbreitung der Elektron-Loch-Plasmen bestimmt.

\section{Ladungsträgerrekombination}

Die Rekombination der Ladungsträger in EHP-s erfolgt über drei wesentliche Mechanismen:

- Rekombination an Störstellen,

- bimolekulare bzw. Plasmarekombination und

- Auger-Rekombination.

Bei der bimolekularen Rekombination rekombiniert ein Elektron mit einem Loch unter Emission eines Photons (strahlende Rekombination). Die Auger-Rekombination ist dagegen ein strahlungsloser Prozeß, bei dem ein Elektron mit einem Loch rekombiniert und die Energie (und der Impuls) an ein Elektron (Leitungsband Augerprozeß) oder Loch (Valenzband Augerprozeß) abgegeben wird.

Aufgeteilt nach den drei Rekombinationsmechanismen erhält man für die Rekombinationsrate $R$ in Abhängigkeit von der Elektron- und Loch-Teilchendichte $n=n_{e}=n_{h}$ Str93:

$$
R=\frac{d n}{d t}=-A n-B n^{2}-C n^{3}
$$

Hierbei ist $A$ die lineare Rekombinationskonstante der Störstellenrekombination, $B$ die bimolekulare Rekombinationskonstante und $C$ die Auger-Rekombinationskonstante.

Für die in den Experimenten dieser Arbeit verwendeten hohen Anregungsdichten von $n>10^{18} \mathrm{~cm}^{-3}$ wird die Rekombination der Ladungsträger von der nichtlinearen Rekombination (Auger- und Plasmarekombination) dominiert. In [Str93] wurden die Rekombinationskonstanten für GaAs bei $T_{L}=300 \mathrm{~K}$ experimentell mit: $B=1,7 \times 10^{-10} \mathrm{~cm}^{3} \mathrm{~s}^{-1}$ und $C=7 \times 10^{-30} \mathrm{~cm}^{6} \mathrm{~s}^{-1}$ bestimmt. Diese Werte werden später in Kap. 5.2 für die Beschreibung der nichtlinearen Rekombination verwendet.

\section{Ausbreitung von Elektron-Loch-Plasmen}

Bei der optischen Anregung von Halbleitern mit kurzen intensiven Laserpulsen mit Photonenenergien $\hbar \omega>E_{G}$ werden Elektron-Loch-Plasmen mit hohen Dichten und auch hohen räumlichen Dichtegradienten angeregt. Diese Dichtegradienten treten sowohl in lateraler Richtung (parallel zur Probenoberfläche) durch die Fokussierung der Laserpulse als auch senkrecht zur Probenoberfläche auf. Beim letzteren werden die großen Dichtegradienten durch die Beersche Absorption $(n \propto \exp [-\alpha z])$ mit entsprechend hohen 
Absorptionskoeffizienten $\alpha(\hbar \omega)$ erzeugt. So ergibt sich für GaAs (300K) bei einer Photonenenergie von $\hbar \omega \approx E_{G}$ eine Absorptionslänge von $1 / \alpha \approx 1 \mu \mathrm{m}$. Für die Interpretation von Experimenten, bei denen Elektron-Loch-Plasmen auf diese Art und Weise räumlich lokal angeregt werden, ist die Berücksichtigung des Ladungsträgertransportes notwendig und ist Gegenstand zahlreicher Untersuchungen gewesen (siehe z.B. Pil85). Die Ursache einer schnellen Elektron-Loch-Plasma-Ausbreitung (Expansion), insbesondere derjenigen senkrecht zur Oberfläche, ist bis heute nicht vollständig verstanden und Gegenstand kontroverser Diskussionen.

Die Expansion des EHP-s sowohl parallel als auch senkrecht zur Probenoberfläche wurde mit Hilfe zahlreicher experimenteller Methoden wie der zeit- und/oder ortsaufgelösten Lumineszenz [Ste84, Hil92, Cin90, Pol96, Nak92], der Raman Spektroskopie [Tse89], dem Vier-Wellen-Mischen [Fox93, Pet92, Ble96], der laserinduzierten Schallausbreitung Gus97] und mittels Flugzeitmessungen [Mah88, Yoo92, Smi89] untersucht. An dieser Stelle soll der Einfachheit halber nur die EHP-Expansion in direkten Halbleitern diskutiert werden. Es wird zwischen der lateralen Ausbreitung mit in der Regel kleineren Dichtegradienten und der Ausbreitung senkrecht zur Probenoberfläche mit größeren Dichtegradienten unterschieden.

Bei der Untersuchung des lateralen Transportes, insbesondere an Vielfach Quantenfilmen, wurden hohe und dichteabhängige Ausbreitungsgeschwindigkeiten gefunden. Als Ursache für diese dichteabhängige laterale EHP-Expansion werden insbesondere folgende Mechanismen diskutiert:

'Phononen Wind': Die treibende Kraft ist hier eine bei der Ladungsträgerrelaxation entstehende Dichte von Phononen, die von den Ladungsträgern reabsorbiert werden können und dabei ihre Energie übertragen. Die Ausbreitungsgeschwindigkeit des EHP-s ist durch die Ausbreitungsgeschwindigkeit der Phononen auf die Schallgeschwindigkeit $v_{\text {Schall }}$ begrenzt [Smi89]. Im indirekten Halbleiter Si wurde mit Hilfe der sogenannten 'Luminescence-Imaging' Methode dieser Mechanismus für die EHP-Expansion auf einer ns-Zeitskala nachgewiesen Ste84. Allerdings wurden bei der lateralen EHP-Expansion in direkten Halbleitern auch Ausbreitungsgeschwindigkeiten $v>v_{\text {Schall }}$ gefunden [Cin90, Hil92, Fox93, Ble96], die nicht mit diesem Prozeß beschrieben werden können.

'Photon-Recycling': Bei diesem Prozeß kommt es innerhalb des Hochdichte-Plasmas zu einer strahlenden Rekombination der Ladungsträger, wobei die emittierten Photonen außerhalb des Plasmas wieder absorbiert werden und neue Elektron-Loch-Paare erzeugen. Ein solcher Mechanismus wurde von Hillmer [Hil92] für die EHP-Ausbreitung in Quantenfilmstrukturen vorgeschlagen, bei der die Quantenfilme als Wellenleiter fungieren und somit einen effizienten Lichttransport in lateraler Richtung ermöglichen. Dieser PhotonRecycling-Mechanismus wird auch von anderen Autoren Ble96, Cin90, Fox93 als Ursache für den lateralen Transport in Quantenfilmen angegeben. Wie in [Fox93] dargelegt, ist in diesem Photon-Recycling-Mechanismus der Transportstrom nicht proportional zu dem 
Kapitel 2 Theoretische Grundlagen und Motivation

Dichtegradienten und läßt sich somit nicht mit einer Diffusionsgleichung beschreiben.

Während die laterale Elektron-Loch-Plasma-Expansion in Quantenfilmen mit dem Photon-Recycling-Mechanismus beschrieben werden kann, ist die Ursache der EHPExpansion senkrecht zur Oberfläche bis heute nicht eindeutig geklärt. Im direkten Vergleich zum lateralen Transport wurde in InP bei $T=300 \mathrm{~K}$ ein wesentlich schnellerer Transport senkrecht zur Oberfläche gefunden [Tse89]. Für die Beschreibung dieses experimentellen Befundes haben die Autoren einen zusätzlichen Driftterm in die Diffusionsgleichung phänomenologisch eingeführt. Ein solcher Driftterm wurde auch in [Mah88] für die Beschreibung der EHP-Expansion in $\mathrm{Ga}_{x} \mathrm{Al}_{1-x} \mathrm{As}$ verwendet. Die zugrunde liegende treibende Kraft ist bei dieser Beschreibung jedoch nicht eindeutig identifiziert. Ein Grund dafür, weshalb die EHP-Expansion senkrecht zur Oberfläche weniger gut verstanden ist, liegt in der Tatsache, daß die meisten Experimente nur sensitiv auf die zeitliche Entwicklung der Ladungsträgerdichte auf der Seite der Probe sind, auf der die Ladungsträger angeregt wurden [Pol96, Nak92, Tse89, Gus97]. Direkte Schlußfolgerungen auf die Ausbreitungsgeschwindigkeit des EHP-s erlauben diese Messungen jedoch nicht, da die zeitliche Entwicklung der Ladungsträgerdichte an der Probenoberfläche nicht nur durch den Ladungsträgertransport, sondern auch durch alle Prozesse der Ladungsträgerrekombination, insbesondere auch durch die Oberflächenrekombination [Mah88, beeinflußt wird. In Flugzeitmessungen, in denen ein Zweifachquantenfilm als lokale Ladungsträgersonde eingesetzt wurde [Hil86], ist nur eine zeitliche Auflösung von $>200$ ps erzielt worden. Die Auflösung wurde begrenzt durch den Prozeß des Ladungsträgereinfangens im Quantenfilm und die endliche Lebensdauer der Zustände im Quantenfilm. Somit können aus diesem Experiment keine Aussagen über die anfänglichen Ausbreitungsgeschwindigkeiten des EHP-s für $\tau<200 p s$ getroffen werden.

Ziel der hier vorliegenden Arbeit ist es nun, die zeitliche Entwicklung der Ausbreitungsgeschwindigkeit des Elektron-Loch-Plasmas in GaAs senkrecht zur Oberfläche mit einer hohen zeitlichen Auflösung $<1$ ps und räumlichen Auflösung $<1 \mu m$ zu untersuchen, um daraus Schlußfolgerungen über die treibende Kraft der Plasmaexpansion ziehen zu können. 


\section{Kapitel 3}

\section{Flugzeitmeßmethode}

Nachdem im vorherigen Kapitel die Motivation für die hier vorliegende Arbeit dargelegt wurde, sollen in diesem Kapitel die für die Untersuchungen verwendeten Meßmethoden vorgestellt werden. Im ersten Abschnitt wird auf die Methode der Anregungs- und Testexperimente und insbesondere auf die Messung der differentiellen Transmission $(\Delta T)$ und Reflexion $(\Delta R)$ und deren Interpretation bei einer räumlich homogenen Anregung der Probe eingegangen. Die Interpretation wird anschließend auf eine inhomogene Anregung der Probe erweitert und die im Rahmen dieser Arbeit entwickelte Flugzeitmeßmethode mit einer zeitlichen Auflösung von 100fs und einer räumlichen Auflösung von $\sim 150 \mathrm{~nm}$ vorgestellt.

\subsection{Anregungs- und Testexperimente}

Eine Standardmethode der nichtlinearen Ultrakurzzeitspektroskopi€ $\S^{1}$ ist die sogenannte Anregungs- und Testmethode. Hierbei regt man die Probe mit einem intensiven Anregungs- bzw. Pumppuls an. Die Veränderung der optischen Eigenschaften der Probe werden von dem mit einer zeitlichen Verzögerung $\tau$ einfallenden Test- bzw. Probepuls ausgetestet (siehe Abb. 3.1). Die Einstellung der zeitliche Verzögerung $\tau$ geschieht über die Änderung der optischen Weglänge $\mathrm{d}(\tau=d / c$; c: Lichtgeschwindigkeit) mit Hilfe eines Verschiebeschlittens.

Die Zeitauflösung dieser Methode ist durch die Pulsdauer $\tau_{\text {puls }}$ beschränkt. Man kann nun zum einen die Änderung der Intensität der transmittierten Probepulse $(\Delta T)$ und zum anderen die Änderung der Intensität der reflektierten Probepulse $(\Delta R)$ in Abhängigkeit der Verzögerungszeit $\tau$ bestimmen. Die Detektion erfolgt i. Allg. zeitintegriert und zum Teil mit zusätzlicher spektraler Auflösung.

Für die Interpretation der Meßkurven $\Delta T(\tau)$ und $\Delta R(\tau)$ betrachtet man die Ände-

\footnotetext{
${ }^{1}$ Einen guten Überblick über die Ultrakurzzeit-Meßmethoden findet man u.a. in Die96.
} 


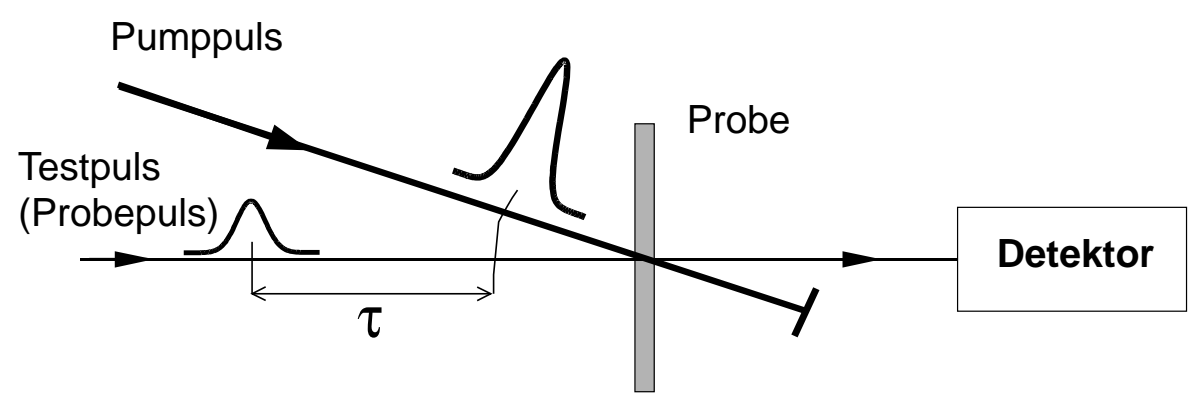

Abbildung 3.1: Prinzip eines nichtlinearen Anregungs- und Testexperimentes

rung der linear optischen Eigenschaften durch die Einwirkung des Pumppulses 2 . Die linear optischen Eigenschaften der Probe (ohne Einwirkung des Pumppulses) bei einer Photonenenergie $\hbar \omega$ können u.a. mit dem komplexen Brechungsindex

$$
\tilde{n}_{0}(\omega)=n_{0}(\omega)+i \cdot \kappa_{0}(\omega)
$$

mit dem Brechungsindex $n$ und dem Extinktionskoeffizienten $\kappa$ beschrieben werden [Woo72. Der Pumppuls verursacht eine von der Verzögerungszeit $\tau$ abhängige Änderung des komplexen Brechungsindex $\Delta \tilde{n}(\omega, \tau)$. An dieser Stelle soll zunächst von einer räumlich konstanten Änderung von $\tilde{n}$ ausgegangen werden: $\Delta \tilde{n}(\vec{r})=$ konst. Im folgenden werden die Probeneigenschaften zu einer festen Verzögerungszeit $\tau$ betrachtet, ohne daß dies explizit erwähnt wird.

\subsubsection{Differentielle Transmission $\Delta T$ und Reflexion $\Delta R$}

Die Transmission $T$ eines schwachen Lichtpulses (Probepuls) mit der Photonenenergie $\hbar \omega$ durch eine Probe mit der Dicke $d$ unter Vernachlässigung von Mehrfachreflexionen wird durch das Beer'sche Gesetz beschrieben:

$$
\begin{aligned}
T(\omega) & =\frac{I_{T}}{I_{0}}=(1-R(\omega))^{2} \cdot e^{-\alpha(\omega) d} \\
\alpha(\omega) & =\frac{4 \pi \kappa(\omega)}{\lambda} \text { Absorptionskoeffizient bei } \omega \\
R(\omega) & =\frac{(n(\omega)-1)^{2}+\kappa(\omega)^{2}}{(n(\omega)+1)^{2}+\kappa(\omega)^{2}} \text { Reflektivität bei } \omega .
\end{aligned}
$$

\footnotetext{
${ }^{2}$ Bei dieser Betrachtung vernachlässigt man eine kohärente Wechselwirkung zwischen den Pump- und Probepulsen (bzw. der von ihnen generierten Polarisation) in der Probe, die zu den in Kap. 2.2 vorgestellten kohärenten Effekten wie z.B. dem Vierwellenmischen führen. Bei den in unseren Experimenten gewählten Anregungsbedingungen, insbesondere den hohen Anregungsdichten und hohen kinetischen Energien der Ladungsträger, können diese Effekte aufgrund der schnellen Dephasierung (siehe Kap. 2.2) vernachlässigt werden.
} 
Hierbei sind $I_{0}, I_{T}$ die Intensitäten der einfallenden bzw. transmittierten Pulse und $\lambda$ die Lichtwellenlänge im Vakuum.

Der Faktor $(1-R)^{2}$ beschreibt die Reflexionsverluste an Vorder- und Rückseite der Probe. Die Änderung der Transmission $\Delta T=T-T_{0}$ sowie die der Reflexion $\Delta R=R-R_{0}$ kann man nun auf die Änderung der optischen Konstanten $\Delta n$ und $\Delta \kappa$ zurückführen. Setzt man für $n_{0}$ und $\kappa_{0}$ die Werte für GaAs bei $T=300 \mathrm{~K}$ und $\hbar \omega=1,5 \mathrm{eV}$ ein Asp83, so erhält man bei einer Probendicke von $3 \mu \mathrm{m}$ :

$$
\begin{aligned}
& \frac{\Delta T}{T_{0}}=-0,004 \frac{\Delta n}{n_{0}}-\mathbf{0 , 5} \frac{\Delta \kappa}{\kappa_{\mathbf{0}}} \\
& \frac{\Delta R}{R_{0}}=\mathbf{0 , 1} \frac{\Delta \mathbf{n}}{\mathbf{n}_{\mathbf{0}}}+0,005 \frac{\Delta \kappa}{\kappa_{0}}
\end{aligned}
$$

Man erkennt, daß die Änderung der Transmission $\Delta T$ von der Änderung des Extinktionskoeffizienten $\Delta \kappa$ und die Änderung der Reflexion $\Delta R$ von der Änderung des Brechungsindex $\Delta n$ dominiert wird.

Die Änderung des Extinktionskoeffizienten $\Delta \kappa$ durch optisch angeregte Ladungsträger wird durch den Effekt des Bleichens aufgrund von Phasenraumfüllung dominiert [Sha99]. Der Zusammenhang zwischen den Besetzungszahlen der optisch gekoppelten Zustände der Elektronen $f_{e}$ und Löcher $f_{h}$ mit dem Extinktionskoeffizienten kann für diesen Prozeß wie folgt beschrieben werden:

$$
\kappa=\kappa_{0} \cdot\left[\left(1-f_{e}\right)\left(1-f_{h}\right)-f_{e} f_{h}\right]=\kappa_{0} \cdot\left(1-f_{e}-f_{h}\right)
$$

Die Terme $\left(1-f_{e}\right)$ und $\left(1-f_{h}\right)$ berücksichtigen, daß schon besetzte Zustände aufgrund des Pauliprinzips nicht noch einmal besetzt werden können und somit die Wahrscheinlichkeit der Absorption eines Photons sinkt. Der Summand $-f_{e} f_{h}$ berücksichtigt, daß mit zunehmenden Besetzungszahlen die Wahrscheinlichkeit der induzierten Emission eines Photons steigt. Wesentlich bei diesem Prozeß ist, daß die Besetzungszahlen $f_{e}$ und $f_{h}$ additiv zur Änderung von $\kappa$ und somit zu $\Delta T$ beitragen, also man auch ein Signal erhält, falls nur Elektronen oder nur Löcher bei der Energie vorhanden sind, die vom Probepuls getestet werden. Im Gegensatz dazu ist die Intensität der Photolumineszenz proportional zu $f_{e} \cdot f_{h}$ [Sha99]. Bei dieser Methode müssen also Elektronen und Löcher gleichzeitig bei dem optisch gekoppelten Übergang vorhanden sein.

Zusammenfassend kann man feststellen, daß die differentielle Transmission $\Delta T$ über den Effekt des Bleichens durch Phasenraumfüllung sensitiv auf Ladungsträger ist, die sich energetisch in der Nähe des vom Probepuls getesteten Übergangs befinden.

Die Änderung des Reflexionskoeffizienten $\Delta R$ wird nach Gleichung 3.3 von der Änderung des Brechungsindex bestimmt. Der Zusammenhang zwischen den Ladungsträgerbesetzungsfunktionen $f_{e}, f_{h}$ und der Änderung des Brechungsindex bzw. des Reflexionsvermögens läßt sich nicht mit einer einfachen Beziehung wie Gleichung 3.4 angeben. Dies 
hat seine Ursache darin, daß der Brechungsindex im Gegensatz zum Extinktionskoeffizienten bei einer Frequenz $\omega_{0}$ nicht nur von den optischen Resonanzen mit der Resonanzfrequenz $\omega_{0}$, sondern auch von Resonanzen mit Resonanzfrequenzen weit entfernt von $\omega_{0}$ bestimmt wird 3 .

Der Zusammenhang von Ladungsträgerbesetzungsfunktion und $\Delta R$ wurde erstmalig von C.V. Shank 1978 betrachtet Sha78]. Mit der Messung von $\Delta R(\tau)$ konnte er Aussagen über die energetische Relaxation von Ladungsträgern treffen. 1992 hat G. Böhne diesen Zusammenhang näher untersucht [Böh92]. An dieser Stelle sollen nur die für diese Arbeit relevanten Ergebnisse dieser Betrachtung vorgestellt werden. Hierbei soll unterschieden werden zwischen dem Einfluß der Ladungsträgerbesetzungsfunktion auf Vorzeichen und Betrag der Reflexionsänderung:

- Vorzeichen: Es ist $\Delta \mathrm{R}<(>)$, wenn der Schwerpunkt der generierten Ladungsträgerverteilung oberhalb (unterhalb) von $E_{\text {probe }}$ liegt:

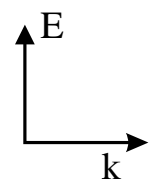

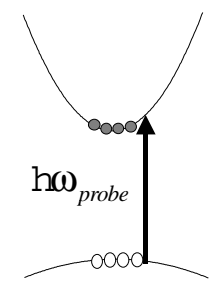

$\Delta \mathbf{R}>\mathbf{0}$

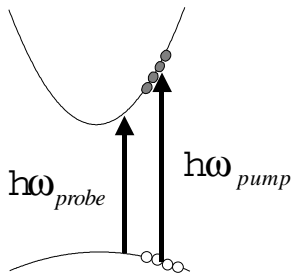

$\Delta \mathbf{R}<\mathbf{0}$

Abbildung 3.2: Zusammenhang Ladungsträgerbesetzung und Vorzeichen von $\Delta R$

- Betrag: In $|\Delta R|$ gehen die Verteilungsfunktionen von Elektronen und Löchern in den Valenzbändern bzw. im Leitungsband, gewichtet mit der jeweiligen kombinierten Zustandsdichte, linear und additiv ein. Damit ist $|\Delta \mathrm{R}|$ abhängig von der Anzahl der beitragenden Ladungsträger. Je mehr Elektron-Loch-Paare sich bei identischer Verteilung oberhalb bzw. unterhalb der Probeenergie befinden, desto größer ist die absolute Änderung im Reflexionsvermögen.

Für die Interpretation von $\Delta R(\tau)$ Messungen bedeutet das, daß man über das Vorzeichen von $\Delta R$ bei geeigneter Wahl von Pump- und Probephotonenenergien Aussagen über die energetische Relaxation der Ladungsträger machen kann und bei einer gegebenen Verteilungsfunktion der Ladungsträger das $\Delta R$ Signal proportional zur Ladungssträgerdichte ist. Diese Tatsache ist die Grundlage für die in dieser Arbeit durchgeführten Untersuchungen.

\footnotetext{
${ }^{3}$ siehe $n, \kappa$ eines harmonischen Oszillators z.B. in Woo72]
} 


\section{2 $\Delta R$ und $\Delta T$ in cis und trans Geometrie}

Im vorherigen Abschnitt ist bei der Intepretation der Anregungs-und Testexperimente von einer räumlich homogenen Ladungsträgeranregung ausgegangen worden. Eine solche möglichst homogene Anregung versucht man auch zu realisieren, wenn man die Ladungsträgerrelaxation untersuchen will, ohne daß die Interpretation durch mögliche Transportvorgänge erschwert wird. Ist nun der Transport der Ladungsträger Gegenstand der Untersuchungen ist man bestrebt, die Ladungsträger möglichst lokal anzuregen und auch zu detektieren. Man unterscheidet nun prinzipiell zwischen lateralen Ladungsträgertransport parallel zur Probenoberfläche und dem Ladungsträgertransport senkrecht zur Oberfläche (siehe Abb. 3.3).
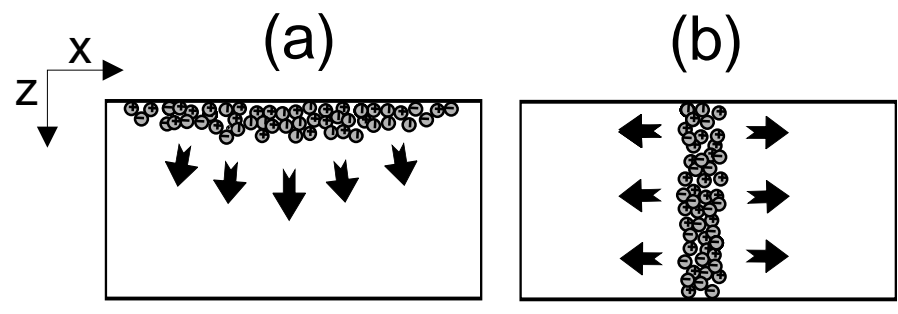

Abbildung 3.3: Ladungsträgertransport (a) senkrecht und (b) parallel zur Probenoberfläche

Für die Untersuchung des lateralen Ladungsträgertransportes wurden mehrere Methoden eingesetzt. Die höchste laterale Auflösung von ca. 100nm wird hierbei mit Hilfe der optischen Nahfeldmikroskopie (SNOM) erreicht (siehe z.B. Gre95, Ent00]). Eine zeit- und ortsaufgelöste Pump- und Probespektroskopie ist mit Hilfe dieser Technik unter Verwendung von nur einer SNOM-Spitze jedoch nur eingeschränkt möglich (siehe z.B. Gro98]). Eine weitere Methode besteht in der Messung der zeit- und ortsaufgelösten Messung der differentiellen Transmission $\Delta T(\tau, x)$ Die Ortsauflösung erreicht man, indem man Pump- und Probestrahl mit Hilfe eines Mikroskopobjektives auf die Probe fokussiert und den Fokus vom Probestrahl relativ zum Fokus des Pumpstrahles verfährt. S. Grosse hat mit dieser Methode mit einer örtlichen Auflösung von 1,4 $\mathrm{m}$ und zeitlichen Auflösung von 150 fs den diffusiven Exzitonentransport in verspannten InGaAs/GaAsP Vielfach-Quantenfilmen untersucht Gro98. Eine deutliche Erhöhung der Auflösung wurde durch den Einsatz einer Festkörperimmersionslinse, welche direkt auf der Probe aufliegt erreicht Vol99. Durch den Einsatz einer GaP-Linse mit einem Brechungsindex $n=3,16$ wird eine laterale Auflösung von $355 \mathrm{~nm}$ erreicht.

Im Rahmen dieser Arbeit soll nun nicht der laterale Transport sondern der Ladungsträgertransport senkrecht zur Oberfläche untersucht werden (siehe Abb. 3.3). Hierzu wurde von uns eine Meßmethode entwickelt, mit der man direkt den Ladungsträgertransport mit einer örtlichen Auflösung von $\sim 150 \mathrm{~nm}$ und zeitlichen Auflösung von $\sim$ 100fs untersuchen kann. Für die räumlich lokale Anregung (lokal in der Tiefe) wird das 
Beer'sche Absorptionsgesetz ausgenutzt. Hierbei nimmt die Ladungsträgerdichte $n$ in die Tiefe der Probe mit $n \propto \exp (-\alpha z)$ ab. In GaAs erreicht man bei einer Photonenenergie von $\hbar \omega=2,4 \mathrm{eV}$ eine Absorptionslänge von $1 / \alpha \approx 100 \mathrm{~nm}$.

Für die räumlich lokale Detektion der Ladungsträger wird die unterschiedliche örtliche Sensitivität der differentiellen Reflexion $\Delta R$ und Transmission $\Delta T$ ausgenutzt:

- $\Delta T$ : Bei der Annahme eines in Richtung z variierenden Absorptionskoeffizienten $\alpha(z)$ ergibt sich für Messung in Transmission T:

$$
T=(1-R)^{2} \cdot \exp \left[\int_{0}^{d}-\alpha(z) d z\right]
$$

Es findet also eine örtliche Mittelung über die Probendicke $d$ statt. Aus der Messung von $\Delta T$ erhält man also keinerlei Information wo sich die Ladungsträger innerhalb der Dicke der Probe befinden.

- $\Delta R$ : In absorbierenden Medien ist die differentielle Reflexion nur sensitiv auf eine Änderung der optischen Konstanten $\tilde{n}(z)$ nahe der reflektierenden Oberfläche. Dieses hat seine Ursache darin, daß die Information über eine Änderung der optischen Konstanten in einem Abstand $a$ von der Oberfläche von dem Probepuls übertragen wird, welcher aber innerhalb der Weglänge $2 a$ auf $\exp \left(-2 \alpha_{\text {probe }} a\right)$ abgeschwächt wird. Dieses ist in Abbildung 3.4 illustriert. Dargestellt ist die Reflexionsänderung $\Delta R$ aufgrund einer deltaförmige Schicht, welche sich in einem Abstand $a$ von der Oberfläche befindet. Die kosinusförmige Modulation wird von der Interferenz des an der Oberfläche und des an dieser deltaförmigen Schicht reflektierten Lichtes verursacht (Vielfachreflexionen wurden hier vernachlässigt). Man erkennt, daß die Einhüllende des $\Delta R$ Signals auf einer Längenskala abfällt, die der halben Absorptionslänge des Probepulses entspricht, $1 / 2 \cdot 1 / \alpha_{\text {probe }}$.

Diese Betrachtung anhand einer deltaförmigen Schicht kann lediglich für die Abschätzung der räumlichen Sensitivität des $\Delta R$ Signales dienen, ist jedoch nicht

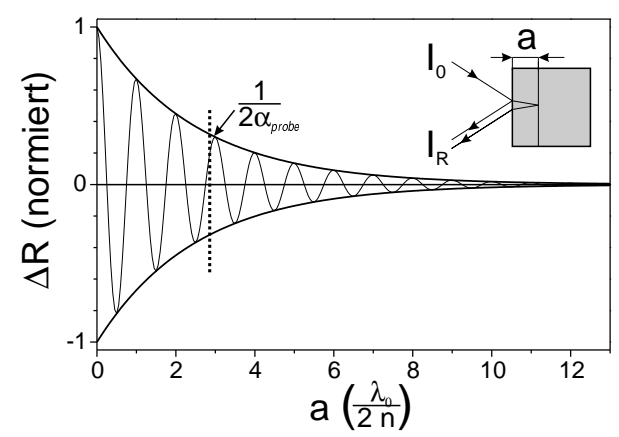

Abbildung 3.4: Reflexionsänderung aufgrund einer deltaförmigen Schicht im Abstand $a$ von der Oberfläche 
für einen direkten Vergleich mit dem Experiment geeignet, da dort die Änderung des komplexen Brechungsindex $\Delta \tilde{n}(z)$ eine kontinuierliche Funktion der Ortskoordinate $(\mathrm{z})$ ist. Eine Möglichkeit, aus einem gegebenen Profil von $\Delta \tilde{n}(z)$ die Reflexionsänderung $\Delta R$ näherungsweise zu bestimmen, besteht darin, die kontinuierliche Funktion $\Delta \tilde{n}(z)$ durch dünne Schichten $(\delta z \ll \lambda)$ mit jeweils konstantem $\Delta \tilde{n} \mathrm{zu}$ approximieren. Berechnungen von $\Delta R$ für $\Delta \tilde{n}(z)$ Profile, die vergleichbar mit den im Experiment vorliegenden sind, zeigen, daß die so bestimmte Reflexionsänderung $\Delta R(\Delta \tilde{n}(z))$ vergleichbar ist mit der Reflexionsänderung $\Delta R(\Delta \tilde{n}(z=0)$ unter der Annahme einer räumlich konstanten Änderung des komplexen Brechungsindex [Wit99].

Die differentielle Reflexion $\Delta R$ kann man nun unter Ausnutzung ihrer räumlichen Sensitivität als räumlich lokale Ladungsträgersonde einsetzen. Dieses geschieht in der in dieser Arbeit angewandten Meßmethode folgendermaßen: In einem Anregungs- und Testexperiment werden gleichzeitig die Änderung der Reflexion $\Delta R$ und Transmission $\Delta T$ gemessen. Es werden zwei unterschiedliche Anregungsgeometrien verglichen. In der einen, cis, werden die Pump- und Probepulse von derselben Seite auf die Probe fokussiert. In der anderen Anordnung, trans, werden die Pump und Probepulse von unterschiedlichen Seiten auf die Probe fokussiert (siehe Abb. 3.5.

\section{cis}

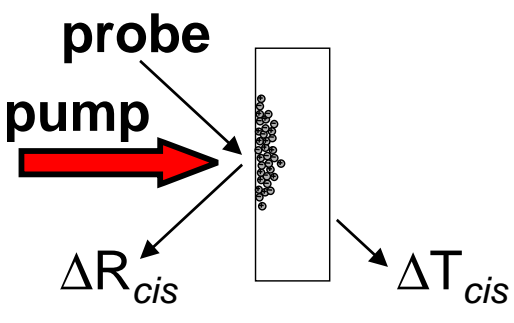

\section{trans}

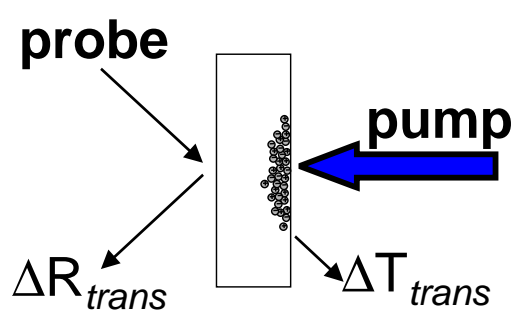

Abbildung 3.5: Pump- und Probe-Geometrien für die Laufzeitmessung cis: Anregung und Detektion auf der Probenvorderseite, trans: Anregung auf der Probenrückseite, Detektion auf der Probenvorderseite

Bei einem Ladungsträgertransport wird nun mit $\Delta R_{\text {cis }}$ die Abnahme der Ladungsträgerdichte auf der Probenseite gemessen, auf der sie generiert wurden und mit $\Delta R_{\text {trans }}$ die Ankunft der Ladungsträger auf der gegenüberliegenden Probenseite. Aus dem Vergleich von $\Delta R_{\text {cis }}$ und $\Delta R_{\text {trans }}$ kann die Zeit für den Ladungsträgertransport über eine Strecke, die der Probendicke $d$ entspricht, bestimmt werden. Mit der Variation der Probendicke $d$ können Flugzeitkurven, also die Flugzeit $t$ als Funktion der Strecke $d$, aufgenommen werden.

Die Differentiellen Transmission $\Delta T$ sollte in cis und trans Geometrie identisch sein 
und stellt somit eine vom Ladungsträgertransport unabhängige Referenz dar, die nur auf die energetische Relaxation der Ladungsträger sensitiv ist.

Die örtliche Sensitivität des $\Delta R$ Signals wurde erstmals in [Bro87] für eine Flugzeitmeßmethode ausgenutzt. Dort wurde mit Hilfe der Messung von $\Delta R$ in cis und trans Geometrie der Wärmetransport in dünnen $(50 \mathrm{~nm} \leq d \leq 300 \mathrm{~nm})$ Goldschichten untersucht.

\subsection{Erhöhung der Ortsauflösung durch Testen des soh-c Überganges}

Aufgrund der Tatsache, daß die örtliche Auflösung von der Absorptionslänge der Probepulse abhängig ist, ist eine geeignete Wahl der Probephotonenenergie $\hbar \omega_{\text {probe }}$ notwendig. Die höchste Empfindlichkeit auf thermalisierte Ladungsträger erreicht man bei einer Probephotonenenergie: $\hbar \omega_{\text {probe }} \sim E_{\text {gap }}$. Dort wird der Übergang von dem Schwerloch (hh) und Leichtlochband (lh) zum Leitungsband (c) in GaAs getestet (siehe Abb. 3.6(a)). Die örtliche Auflösung von $\frac{1}{2}\left(1 / \alpha_{\text {probe }}\right) \approx 0,5 \mu \mathrm{m}$, die man hier erreicht, ist eher gering. Eine signifikante Erhöhung der Ortsauflösung auf 150nm kann man durch die Wahl der Probephotonenenergie in der Nähe des Übergangs vom Spin-Bahn abgespaltenen Lochbandes (soh) zum Leitungsband (c) erreichen (siehe Abb. 3.6(b)):

$$
\hbar \omega_{\text {probe }} \approx E_{g}+\Delta_{\text {soh }} .
$$

$\Delta_{\text {soh }}$ ist hierbei der energetische Abstand zwischen soh-und hh- bzw. lh-Band bei $\vec{k}=0$. Bei einer solchen Wahl der Probephotonenenergie nutzt man die Tatsache aus, daß bei der Abhängigkeit der $\Delta T$ und $\Delta R$ Messungen von der Ladungsträgerverteilungsfunktion die Besetzungsfunktionen der Elektronen $f_{e}$ und der Löcher $f_{h}$ additiv beitragen (siehe Kap. 3.1). Bei einer genügend geringen Pumpphotonenenergie $\hbar \omega_{\text {pump }}$ ist das soh Band nämlich unbesetzt $\left(f_{\text {soh }}=0\right)$. Der Hauptbeitrag für die $\Delta R$ und $\Delta T$ Signale resultiert hier also nur von den Elektronen im Leitungsband. Erstmals wurde die Technik des Austestens des soh-c Übergangs von Alexandrou [Ale95] vorgestellt. In einer Messung der differentiellen

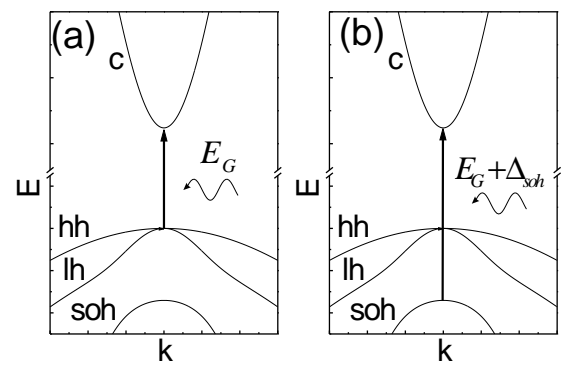

Abbildung 3.6: Bandstruktur von GaAs mit den optischen Übergangen (a) hh/lh-c und (b) soh-c 
Transmission wurde diese Technik ausgenutzt, um gezielt nur die Dynamik der Elektronen zu untersuchen und kohärente Effekte zu unterdrücken. Diese Technik wurde bisher noch nicht in $\Delta R$ Messungen verwendet. Daher muß in einer Voruntersuchung die Möglichkeit der Nutzung dieser Methode in $\Delta R$ Messungen gezeigt werden (siehe Kap. 4.4.3). Die Tatsache, daß diese Methode nur sensitiv auf die Dynamik der Elektronen ist, spielt für die Transportuntersuchungen dieser Arbeit keine Rolle da hier, der ambipolare d.h. der gemeinsame Transport von Elektronen und Löchern untersucht werden soll.

Mit der Messung der differentiellen Reflexion $\Delta R$ und Transmission $\Delta T$ in cis und trans Geometrie bei einer Probephotonenenergie von $\hbar \omega_{\text {probe }} \approx E_{g}+\Delta_{\text {soh }}$ steht nun eine Laufzeituntersuchungsmethode zur Verfügung, welche sowohl eine hohe räumliche $(\sim 150 \mathrm{~nm})$ als auch zeitliche Auflösung ( 100fs) besitzt. 


\section{Kapitel 4}

\section{Experimentelle Realisierung}

Nachdem im vorherigen Kapitel die Idee der Flugzeitmeßmethode vorgestellt wurde, soll im folgenden die konkrete experimentelle Realisierung dargelegt werden. Im ersten Teil wird die Erzeugung und Charakterisierung der im Experiment verwendeten unterschiedlichen fs-Pulse diskutiert. Im zweiten Abschnitt werden die untersuchten GaAs Proben vorgestellt, bevor im dritten Abschnitt auf den konkreten Versuchsaufbau eingegangen wird. Im Anschluß daran werden erste Experimente vorgestellt, mit denen die Anwendbarkeit der Meßmethode untersucht werden soll.

\subsection{Das Lasersystem}

Das für alle Experimente verwendete Lasersystem besteht aus einem Ti:Saphir Oszillator, regenerativen Verstärker $\left(\operatorname{Reg} A^{1}\right)$ und einem optisch parametrischen Verstärker $\left(\mathrm{OPA}{ }^{2}\right)$ und erzeugt $n J-\mu J$ fs- Pulse mit durchstimmbaren Photonenenergien (siehe Abb. 4.1). Als Quelle des Verstärkersystems dient ein selbst gebauter Ti:Saphir Oszillator [Fre94],

Repetitionsrate: $\mathrm{f}_{\text {rep }}=250 \mathrm{kHz}$, Pulsdauer: $\tau_{\text {puls }} \sim 50 \mathrm{fs}$

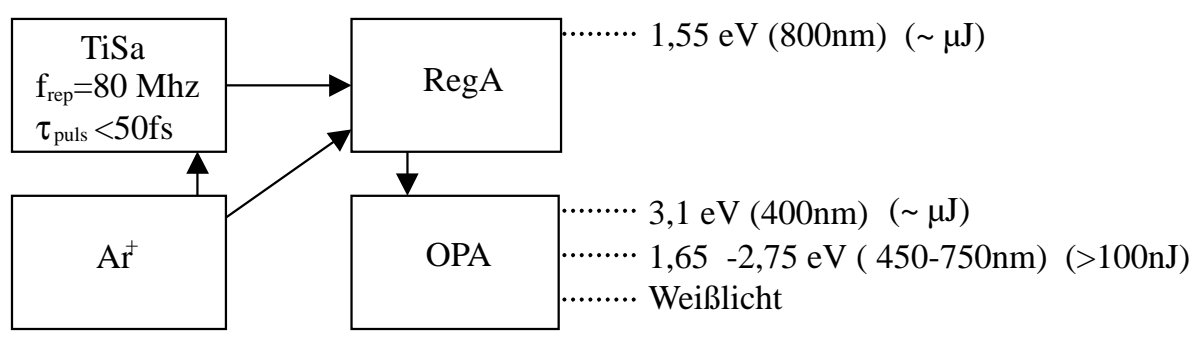

Abbildung 4.1: Schematischer Aufbau des Lasersystems

der von einem Argon-Ionenlaser gepumpt wird. Dieser erzeugt Pulse mit einer Pulslänge $\tau_{\text {puls }}<50 \mathrm{fs}\left(\mathrm{FWHM} \mathrm{S}^{3}\right.$ der Intensität) und Pulsenergie von maximal 5nJ bei einer Repe-

\footnotetext{
${ }^{1}$ Regenarative Amplifier RegA Coherent Model 9050

${ }^{2}$ OPA engl.: optical parametrical amplifier

${ }^{3}$ FWHM engl.: full width at half maximum
} 
titionsrate von $80 \mathrm{MHz}$. Diese Oszillatorpulse werden von einem regenerativer Verstärker nach dem Prinzip des CPA nachverstärkt [Ree94, Ree96]. Hierzu werden die Pulse zuerst von einem Expander zeitlich auseinandergezogen, anschließend verstärkt und schließlich in einem Kompressor zeitlich komprimiert. Man erhält Pulse mit ca. $4 \mu \mathrm{J}$ Pulsenergie bei Pulsdauern von $\tau_{p u l s} \approx 50 \mathrm{fs}$. Die Repetitionsrate ist im Bereich $0 \ldots 300 \mathrm{kHz}$ variabel und wurde für die Messungen, die in dieser Arbeit vorgestellt werden, auf $250 \mathrm{kHz}$ eingestellt. Die Zentralwellenlänge des Verstärkersystems ist im Bereich von 800nm $\pm 30 \mathrm{~nm}$ mit Hilfe des Ti:Saphir Oszillators einstellbar und wurde dem Zweck der einzelnen Messungen angepaßt. Mit den intensiven RegA Pulsen wird ein optisch parametrischer Verstärkei ${ }^{5}$ betrieben [Ree95]. In diesem wird mit etwa 25\% der Pulsenergie via Fokussierung auf einen Saphirkristall ein Weißlichtkontinuum im Spektralbereich von 400 - 1000 nm erzeugt. Die restlichen $75 \%$ der Pulsenergie werden mit Hilfe eines nichtlinear optischen Kristalls (in diesem Fall BBO ${ }^{6}$ frequenzverdoppelt und anschließend als Pumppuls für die optisch parametrische Verstärkung eines Teils des Weißlichtkontinuums eingesetzt. Als Resultat erhält man OPA-Signal Pulse ${ }^{7}$ mit Pulslängen $\tau_{\text {puls }}<100 \mathrm{fs}$ und Pulsenergien $>100 \mathrm{~nJ}$, wobei man die Zentralwellenlänge in dem Bereich 450nm - 750nm durchstimmen kann.

Das OPA RegA Lasersystem stellt zum einen intensive fs Lichtpulse praktisch im gesamten sichtbaren bis zum nahen infrarot Spektralbereich zur Verfügung und ermöglicht außerdem noch Zwei- bzw. Mehrfarbexperimente, bei denen Lichtpulse mit unterschiedlicher Zentralwellenlänge in einem Experiment gleichzeitig eingesetzt werden. Da alle Lichtpulse aus ein und demselben Lichtpuls (RegA Puls) über kohärente Prozesse generiert werden, besitzen die einzelne Pulse untereinander eine feste Phasenbeziehung. Die Verzögerungszeit zwischen den Pulsen ist somit keinerlei Schwankung, dem so genannten Jitter, unterworfen 8 . In dieser Arbeit werden alle hier vorgestellten Laserlichtquellen (RegA Pulse, frequenzverdoppelte RegA Pulse, OPA Signal Pulse und Weißlichtkontinuums Pulse) je nach Versuchsanordnung in unterschiedlichen Kombinationen genutzt.

\subsubsection{Die spektrale Filterung}

Im Rahmen dieser Arbeit erweisen sich folgende spektrale Filterungen als notwendig:

- Aus dem Weißlicht-Kontinuum sollen Pulse mit etwa $30 \mathrm{meV}$ spektraler Breite (FWHM) im Bereich von $1,6 \mathrm{eV} \leq \hbar \omega \leq 2 \mathrm{eV}$ herausgefiltert werden.

- Aus den OPA-Pulsen im sichtbaren Bereich $1,65 \mathrm{eV} \leq \hbar \omega \leq 2,75 \mathrm{eV}$ müssen Restanteile des Weißlichtkontinuums sowie der Grundwelle bei $1,55 \mathrm{eV}$ herausgefiltert werden, um spektral saubere Pulse zu erhalten.

${ }^{4}$ CPA engl. chirped pulse amplification

${ }^{5}$ OPA Coherent Model 9400

${ }^{6}$ BBO: Beta Barium Borat Kristall

${ }^{7}$ Neben den OPA-Signal Pulsen werden bei der parametrischen Verstärkung auch sogenannte IdlerPulse mit $\omega_{\text {Pump }}=\omega_{\text {Signal }}+\omega_{\text {Idler }}$ generiert, wobei $\omega_{\text {Pump }}, \omega_{\text {Signal }}, \omega_{\text {Idler }}$ die Frequenzen der Pump-, Signal- und Idlerpulse sind. Diese wurden jedoch für diese Arbeit nicht verwendet.

${ }^{8}$ Ein solcher Jitter tritt z.B. bei der Generierung von Lichtpulsen mit Hilfe synchronisierter aber unterschiedlicher Laseroszillatoren auf. 
- Aus der Pulsflanke der RegA-Pulse bei $1,55 \mathrm{eV}$ soll ein schmalbandiges Spektrum herausgeschnitten werden.

Bei der spektralen Filterung von fs-Pulsen ist zu beachten, daß die Aufbauten keine oder nur bewußt gewählte Weglängendifferenzen für unterschiedliche Wellenlängen besitzen, damit die Pulse zeitlich kompakt bleiben. Für die drei Anwendungen werden 3 unterschiedliche Anordnungen zur spektralen Filterung eingesetzt:

- ein Prismen-Pulsformer für das Weißlichtkontinuum (siehe Abb. 4.2(b))

- eine Prismen-Dispersionskompensationsstrecke [For84] für die OPA-Signal Pulse (siehe Abb. 4.3) und

- ein Gitter-Pulsformer [Wei88] für die RegA-Pulse (siehe Abb. 4.2(a)).

Bei den Pulsformern wird der einfallende Laserstrahl durch ein Prisma bzw. Gitter spektral aufgespalten. Eine Linse, die im Abstand $f$ (f... Fokus der Linse) von dem Prisma/Gitter positioniert ist, erfüllt zwei Aufgaben. Zum einen fokussiert sie den Laserstrahl für gleiche Wellenlängen auf den Spiegel und zum anderen gleicht sie die spektrale Divergenz aus. In der Ebene des Spiegels wird nun mit einer Maske bzw. mit einem Spalt das gewünschte Spektrum herausgeschnitten. Bei dem Einsatz eines Gitters erreicht man aufgrund der großen Beugungsdivergenz eine höhere spektrale Aufösung. Im Vgl. zum Einsatz eines Prismas ${ }^{9}$ müssen jedoch größere Intensitätsverluste in Kauf genommen werden. Bei exakter Justage sind die Pulsformer nicht dispersiv. Demgegenüber kann man
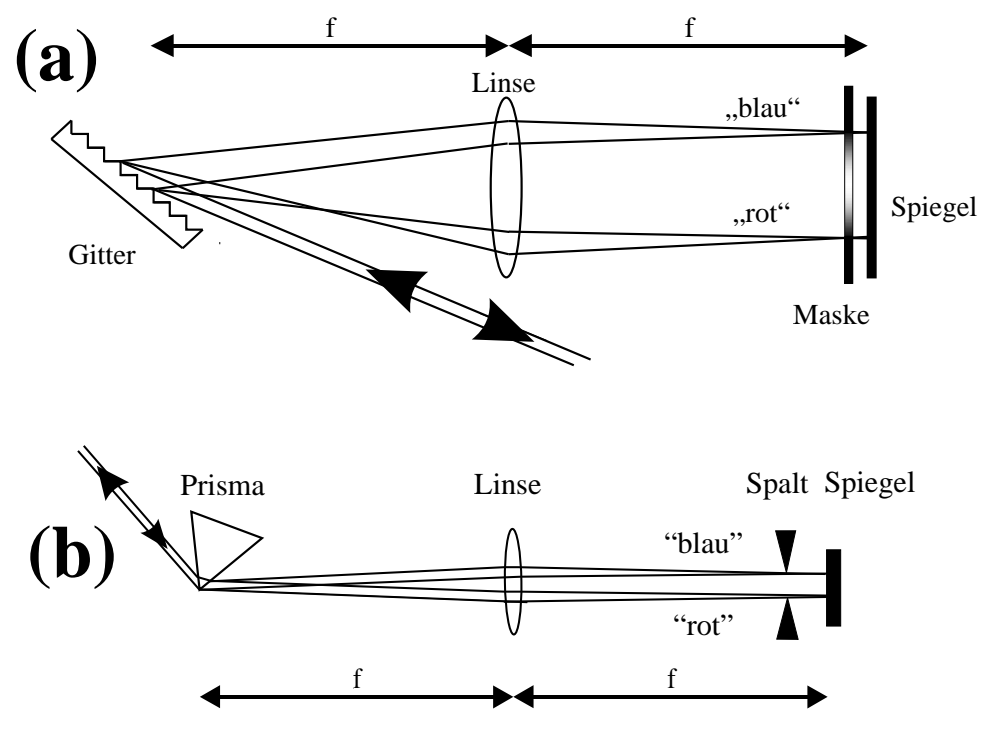

Abbildung 4.2: (a)Gitter-Pulsformer (b)Prismen-Pulsformer

mit Hilfe einer Dispersionskompensationsstrecke (siehe Abb. 4.3) aufgrund unterschiedlicher optischer Weglängen von unterschiedlichen Wellenlängen die eventuell vorhandene

\footnotetext{
${ }^{9}$ Gitter mit 1200 Linien/mm: $\Delta \lambda<1 \mathrm{~nm}$ Prismen aus BK7 66, $9^{\circ}: \Delta \lambda \sim 10 \mathrm{~nm}$ bei $\lambda=800 \mathrm{~nm}$
} 
zeitliche Dispersion der Pulse kompensieren [For84]. Dieses ist bei den OPA-Signal Pulsen notwendig. Gleichzeitig kann man mit Hilfe eines Spaltes den vorhandenen Weißlichtkontinuumsuntergrund sowie die Grundwelle bei $800 \mathrm{~nm}$ herausfiltern.

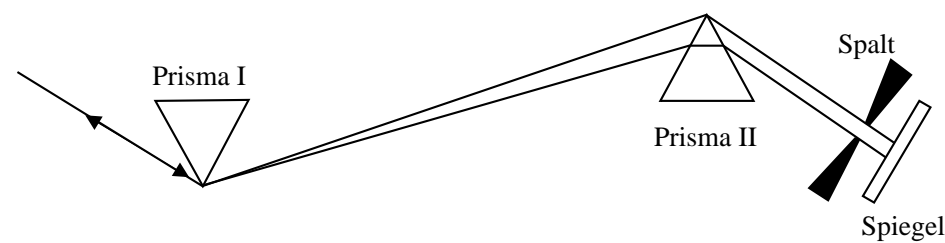

Abbildung 4.3: Prismen Dispersionskompensationsstrecke

\subsubsection{Charakterisierung der Pulse}

Zur Justage des Lasersystems und der Pulsformer werden die Pulsspektren mit Hilfe eines Laserspektrometers ${ }^{10}$ fortlaufend beobachtet. 2 Beispiele für Pulsspektren, wie sie in den Experimenten verwendet werden, sind in Abbildung 4.4 dargestellt: auf der höherenergetischen Seite ein OPA-Puls bei 2,10eV (590nm Zentralwellenlänge) mit 40meV (FWHM) spektraler Breite, sowie ein RegA-Puls bei 1,48eV (833nm Zentralwellenlänge) mit 20meV (FWHM) spektraler Breite. Bei Annahme von gaußförmigen Pulsen entsprechen diese spektralen Breiten Pulsdauern von etwa 50fs bzw. 100fs. Zur weiteren Charakterisierung

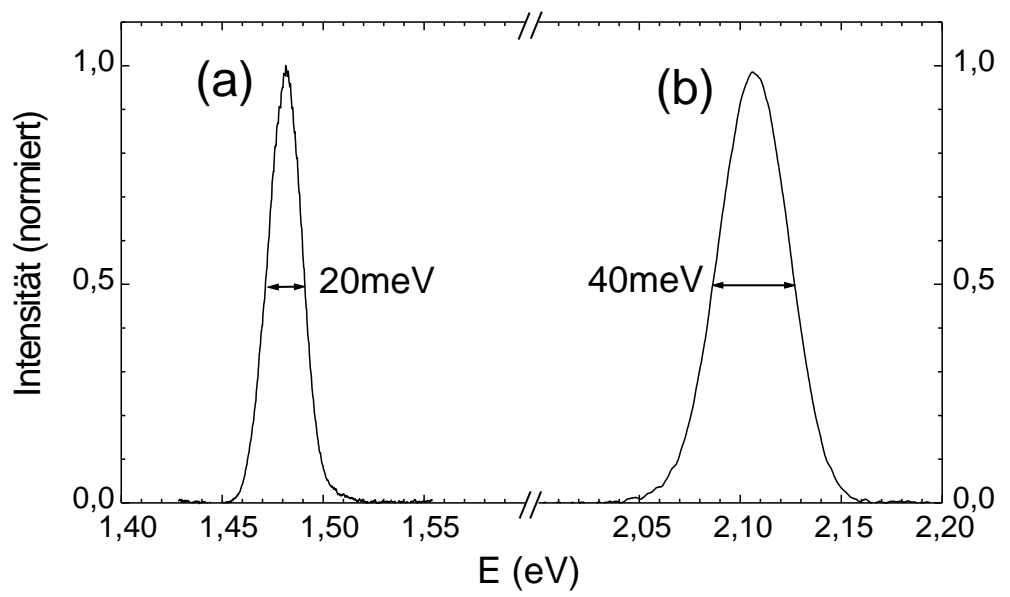

Abbildung 4.4: Intensitätsspektren von (a) RegA Puls nach Pulsformer mit Maske, (b) OPA Signal Puls

wird die Pulslänge mit Hilfe von Standard-Autokorrelationstechniken wie kollineare und nichtkollineare Intensitätsautokorrelation bestimmt. In Abbildung 4.5 ist die nichtkollineare Autokorrelation des OPA-Signal Pulses aus der Abbildung 4.4(b) zusammen mit

${ }^{10}$ Laser Spectrum Analyser ist-rees E201 
einem Fit unter Annahme eines gaußförmigen Pulses mit einer Pulslänge von $\tau_{p u l s}=50 \mathrm{fs}$ (FWHM) dargestellt.

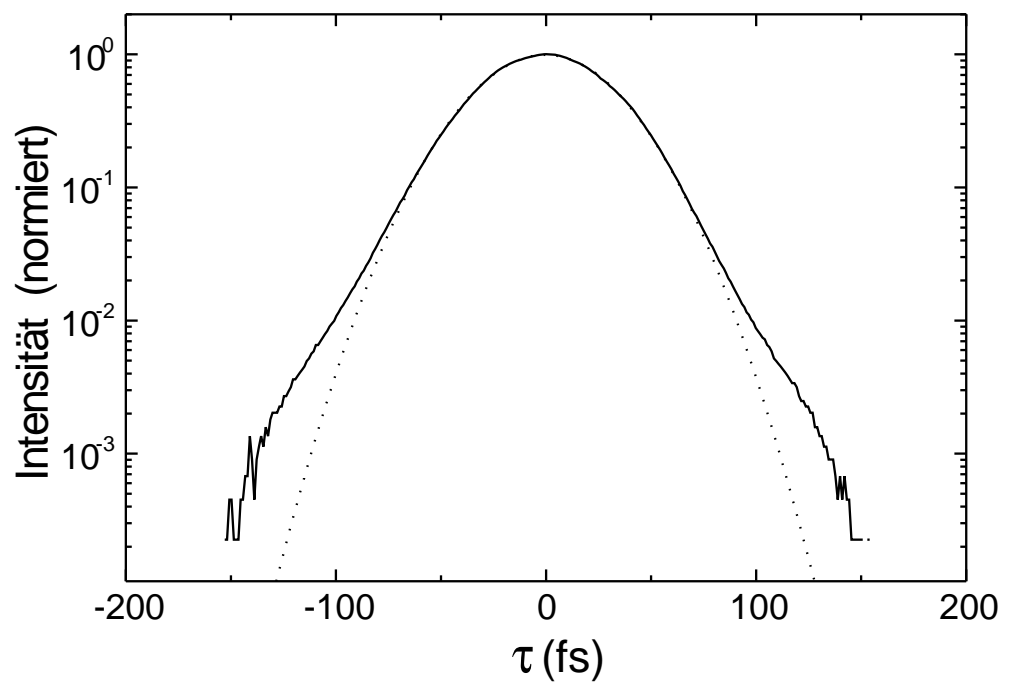

Abbildung 4.5: Nichtkollineare Autokorrelation eines OPA-Signal Pulses (gestrichelte Linie: gaußförmiger Fit mit einer Pulslänge von 50fs (FWHM)) 


\subsection{Die Probenpräparation}

Die Experimente werden an semiisolierendem GaAs (kompensiert dotiertes Wafermaterial der Firma Wacker) durchgeführt. Für die Flugzeituntersuchungen werden Proben mit einer von der lateralen Position abhängigen Dicke $d \sim 1 \mu \mathrm{m}$ benötigt. Bei den in dieser Arbeit verwendeten Proben geschah die Probenpräparation durch Schleifen mit einem Dimpler und einem anschließenden Ätzvorgang mit einer Brommethanollösung ${ }^{11}$. Die Probengeometrie, die man mit dieser Methode erhält, ist durch den Radius der Dimplerscheibe bestimmt (siehe Abb. 4.6). In dieser Arbeit wurden Proben mit einer minimalen Probendicke

(a)

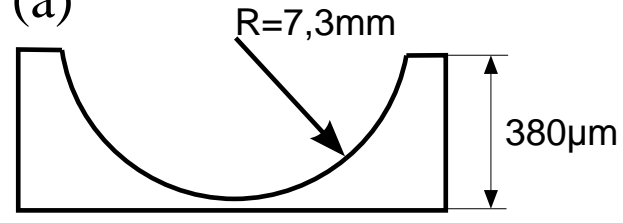

(b)

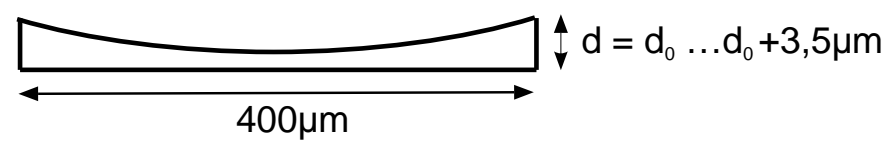

Abbildung 4.6: (a) gedünnte Probe im Schnitt (nicht maßstäblich), (b) der für die Messung relevante Ausschnitt einer gedünnten Probe (etwa maßstäblich)

$0,3 \mu \mathrm{m} \leq d_{0} \leq 0,8 \mu \mathrm{m}$ verwendet. Bei dem im Experiment eingestellten Fokusdurchmesser des Probestrahls von $\sim 15 \mu \mathrm{m}$ ergibt sich eine Dickenvariation über die Fläche des Fokus von $<10 \mathrm{~nm}$. Diese Variation ist klein gegenüber den im Experiment verwendeten Probendicken von $d>0,5 \mu \mathrm{m}$ und kann im Folgenden vernachlässigt werden.

Um eine bessere Wärmeabfuhr bei den Raumtemperaturmessungen zu gewährleisten, wurden die Proben teilweise auf Saphir aufgeklebt.

\subsection{Der Meßaufbau}

Die wesentlichen Teile des Meßaufbaus sind in der Abbildung 4.7 dargestellt12, Für die Messung von $\Delta R$ und $\Delta T$ wird eine Standard Lock-In-Verstärkertechnik genutzt. Hierfür wird der Pumpstrahl mit einer Frequenz von $700 \mathrm{~Hz}$ mit Hilfe eines Choppers moduliert. Die Verzögerungszeit $\tau$ wird mit Hilfe einer Verzögerungsstrecke bestehend aus einem Verschiebschlitten ${ }^{13}$ auf welchem ein Retroreflektor ${ }^{14}$ montiert ist, im Pumpstrahl eingestellt. Anschließend wird dieser auf die Probenvorder- bzw. Rückseite in einem Einfallswinkel von $20^{\circ}$ auf einen Fokusdurchmesser von $\approx 20 \mu \mathrm{m}$ fokussiert. Die optischen Wege auf die Probenvorder -bzw. Rückseite sind abgeglichen, so daß das Umschalten zwischen den beiden Wegen einfach mit Hilfe eines abnehmbaren Spiegels auf einem Magnethalter geschehen kann. Der Probestrahl wird nach dem Durchgang durch einen

\footnotetext{
${ }^{11}$ Für eine detaillierte Beschreibung der Probenpräparation siehe etwa [Sän96.

${ }^{12}$ Der Meßaufbau für die Raumtemperaturmessungen ist detailliert in Wit99 beschrieben.

${ }^{13}$ Schneeberger Schlitten mit ca. $15 \mathrm{~cm}$ nutzbaren Hub. Entspricht einer max. Verzögerungszeit von ca. 1ns.

${ }^{14}$ Newport Breitband Retroreflektor UBBR-1-L, Winkelgenauigkeit: $1^{\prime}$
} 
Strahlteiler $(\mathrm{R} / \mathrm{T}=90 / 10)$ senkrecht auf die Vorderseite der Probe auf ca. 2/3 des Durchmessers des Pumpfokus fokussiert. Der in retro reflektierte Probestrahl wird, nachdem er von der selben Optik wie der einfallende Strahl kollimiert wurde, von dem Strahlteiler auf eine Photodiode gelenkt. Auch der transmittierte Probestrahl wird auf eine Photodiode fokussiert. Um den Einfluß von Laser Intensitätsschwankungen auf die Meßsignale zu reduzieren, werden Photodioden mit entgegengesetzter Polarität eingesetzt, die von einem Teil des Probestrahls bestrahlt werden, um die $\Delta R$ und $\Delta T$ Meßsignale jeweils getrennt abzugleichen. In Verbindung mit zwei Lock-In-Verstärkern können Intensitätsänderungen kleiner $10^{-4}$ detektiert werden. Der örtliche Überlapp zwischen

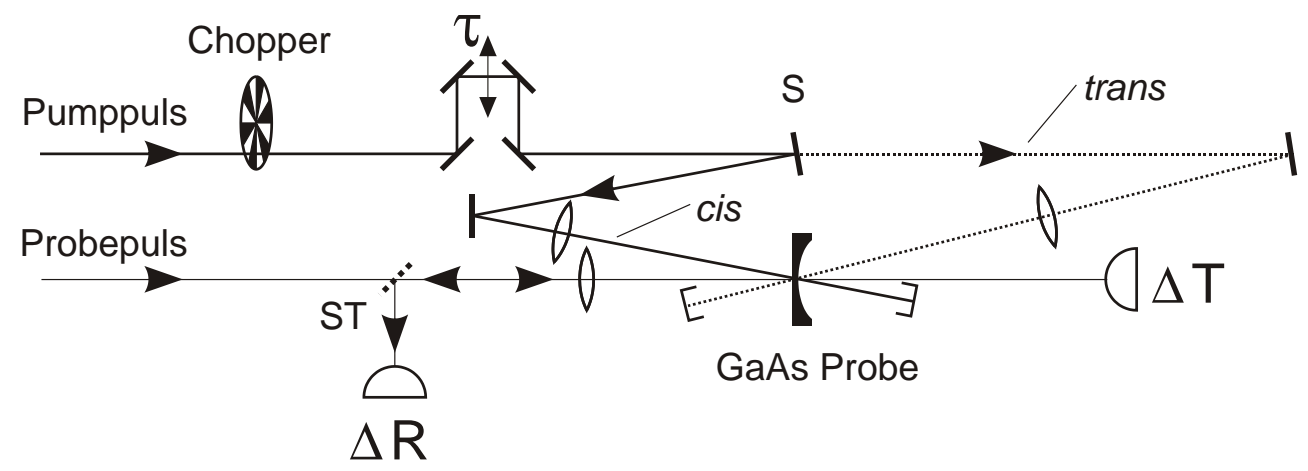

Abbildung 4.7: Schematische Darstellung des experimentellen Aufbaus (S, Spiegel auf Magnethalter; ST, Strahlteiler; $\Delta R, \Delta T$ Photodioden)

den Pump- und Probestrahlfoki wird sorgfältig justiert. Dies geschieht mit Hilfe eines Mikroskopobjektives, welches das Streu-bzw. Lumineszenzlicht von der Probe auf eine CCD Kamera abbildet.

Für die Messung bei tiefen Temperaturen $T_{L}<300 \mathrm{~K}$ wird die Probe in einen HeDurchflußkryostaten ${ }^{15}$ eingebaut, mit welchem sich Temperaturen bis min. 4K einstellen lassen. Aufgrund der kompakten Geometrie des Kryostaten läßt sich dieser in den Aufbau integrieren, ohne wesentliche Veränderungen am Experiment vorzunehmen.

Die Variation der Probendicke $d$ geschieht durch eine Verschiebung der gedünnten Probe (siehe Kap. 4.2 in lateraler Richtung. Die Probendicke $d$ an der jeweiligen Position des Probestrahlfokus auf der gedünnten GaAs Probe wird durch eine präzise Messung der Probestrahltransmission $I / I_{0}$ mit Hilfe eines extra Lock-In Verstärkers gemessen. Hierfür wird der Probestrahl mit einem Chopper moduliert. Mit Hilfe der bekannten optischen Konstanten von GaAs Asp83 bei der Probephotonenenergie $\hbar \omega_{\text {probe }}$ wird die jeweilige Probendicke $d$ bestimmt. Der (systematische) Fehler in der Bestimmung der Probendicke wird mit Hilfe der unabhängigen Bestimmung von $d$ über die Pumpstrahltransmission auf $<10 \%$ abgeschätzt.

\footnotetext{
${ }^{15}$ CryoVac Konti-Kryostat Mikroskop
} 
Bei den in dieser Arbeit durchgeführten nicht entarteten Pump- und ProbeExperimenten (d.h. $\hbar \omega_{\text {probe }} \neq \hbar \omega_{\text {pump }}$ ) ist der genaue Abgleich der optischen Weglängen von Pump und Probestrahl $(\tau=0)$ schwierig. Zum einen sind die optischen Wege, die die Pulse getrennt laufen, z.T. sehr lang $(1-2 \mathrm{~m})^{16}$. Und zum anderen können nur nichtlinear optische Verfahren zum Auffinden von $\tau=0$ benutzt werden. Da für andere Verfahren wie die Erzeugung der zweiten Harmonischen (SHG) oder der Zweiphotonenabsorption [Die96] jeweils ein Umbau des Experimentes notwendig wäre und dies zu einer Veränderung der optischen Wege und somit zu einer Ungenauigkeit der Bestimmung von $\tau=0$ führen würde, hat es sich als sinnvoll erwiesen, die optischen Wege anhand der Anstiegsflanke des $\Delta T(\tau)$ Signals abzugleichen. Dies geschieht mit einer absoluten Genauigkeit von $<200 f s$ und einer relativen Genauigkeit zwischen den beiden Anregungsgeometrien cis und trans von $<50 f s$ 17.

Als Maß für die Intensität der Probenanregung durch den Pumppuls wird in dieser Arbeit die Fluenz $F$ angegeben. Sie ist definiert als Quotient der Pulsenergie $E_{\text {puls }}$ und Fläche des Fokus $A=\pi \sigma^{2}$ mit dem Fokusradius $\sigma$. Der Fokusradius wird aus dem gemessenen Strahlquerschnitt $a$ vor der Fokussierlinse und der Brennweite $f$ der Linse mit Hilfe gaußscher Strahlenoptik bestimmt: $\sigma=\frac{\lambda f}{2 \pi a}$.

Im Gegensatz zur Fluenz, die dem Experimentator direkt zugänglich ist, sind für eine Bestimmung der Anregungsdichte $n$, also der Dichte der angeregten Ladungsträger, Modellvorstellungen notwendig. Deshalb wird lediglich für den Vergleich von experimentellen Ergebnissen mit Modellsimulationen zwischen Ladungsträgerdichte $n$ und Fluenz $F$ umgerechnet.

\subsection{Erste experimentelle Ergebnisse}

Nachdem im vorherigen Abschnitt der konkrete Meßaufbau vorgestellt wurde, sollen nun die Ergebnisse von Experimenten diskutiert werden, die zum Ziel haben, die in Kap. 3 vorgestellten Überlegungen zur Meßmethode experimentell zu verifizieren.

\footnotetext{
${ }^{16}$ z.B. bei der Verwendung der OPA Signal Pulse als Pump und der Weißlichtkontinuumspulse als Probepulse werden die Pulse schon innerhalb des Lasersytems (OPA) getrennt und auf unterschiedlichen Wegen zum Experiment geführt.

${ }^{17}$ Prinzipiell ist bei der Definition von $\tau=0$ bei einer Messung in trans Geometrie zu beachten, daß aufgrund der Tatsache, daß Pump- und Probepulse in entgegengesetzten Richtungen mit einer endlichen Gruppengeschwindigkeit propagieren, sie an unterschiedlichen Stellen der Probe z.B. bei $z=0$ und $z=d$ zu unterschiedlichen Verzögerungszeiten $\tau$ ihren maximalen zeitlichen Überlapp besitzen. Da jedoch die Propagation der optischen Pulse bei den verwendeten Probendicken $d<3 \mu \mathrm{m}$ auf einer Zeitskala $\tau_{\text {prop }} \ll$ $\tau_{\text {puls }}$ stattfindet, die wesentlich kleiner als die Pulsdauer von $\tau_{\text {Puls }} \sim 100 \mathrm{fs}$ ist, kann in dieser Arbeit die so verursachte Unsicherheit in der Bestimmung von $\tau=0$ vernachlässigt werden.
} 


\subsubsection{Abhängigkeit des $\Delta R$ Signals von der energetischen Relaxation der Ladungsträger}

Im Abschnitt 3.1.1 wurde die Abhängigkeit der differentiellen Reflexion $\Delta R$ von der energetischen Ladungsträgerverteilungsfunktion diskutiert. Insbesondere wurde festgestellt, daß ein positives / negatives $\Delta R$ Signal auftreten sollte, falls der Schwerpunkt der Ladungsträgerverteilungsfunktion energetisch unterhalb / oberhalb der vom Probepuls getesteten Übergänge liegt und daß das $\Delta R$ Signal proportional zur Ladungsträgerdichte ist. Um dieses experimentell zu verifizieren, sind $\Delta R$ Messungen an einer GaAs Volumenprobe bei relativ geringen Fluenzen von $F=17 \mu \mathrm{Jcm}^{-2}$ mit einer Probephotonenenergie von $E_{\text {probe }}=1,47 \mathrm{eV}$ und unterschiedlichen Pumphotonenenergien (siehe Abb. 4.8(a)) durchgeführt worden. Sowohl die Pump als auch die Probepulse wurden mit Hilfe von Gitterpulsformern (siehe Kap. 4.1.1) aus RegA Pulsen generiert.
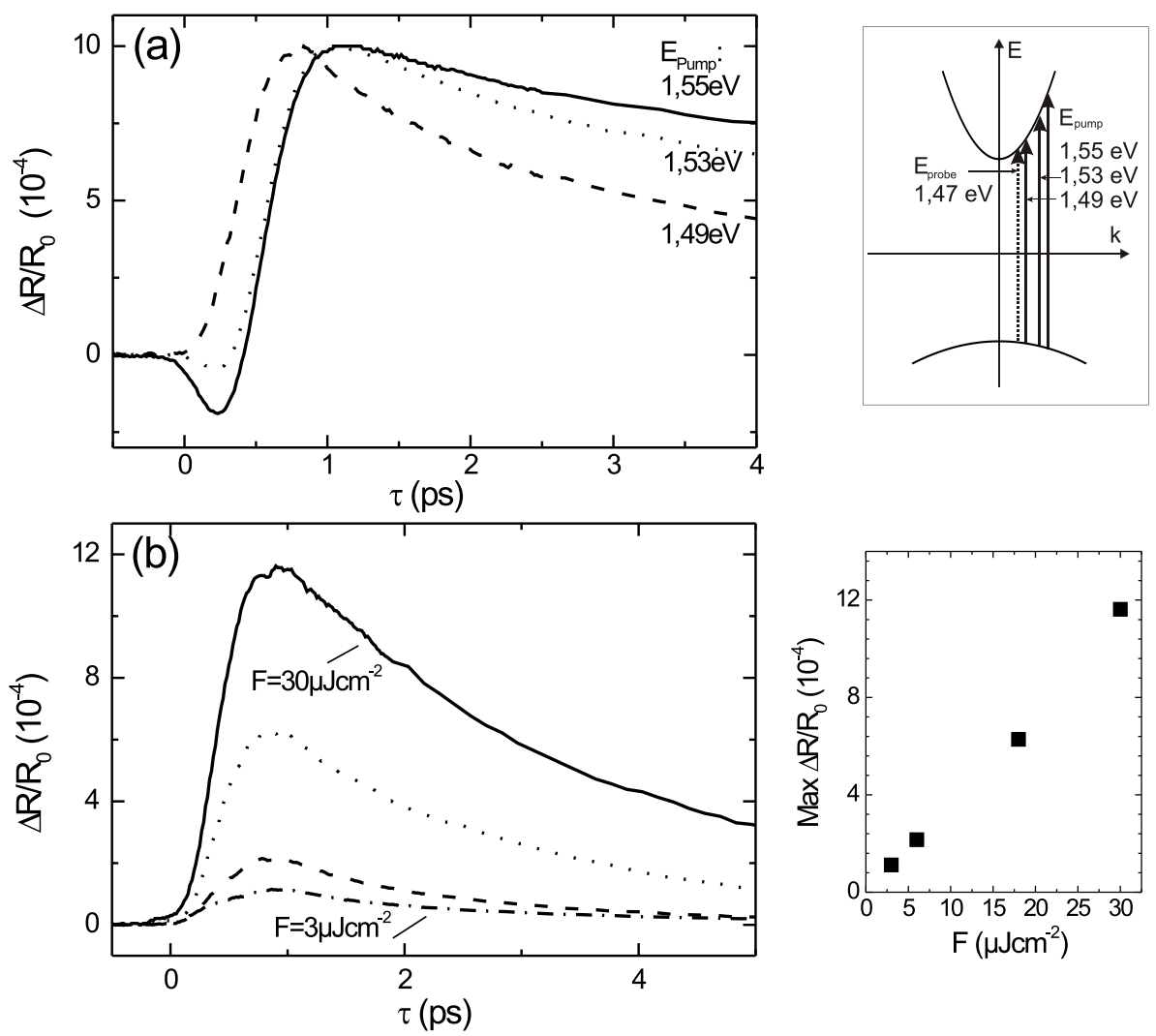

Abbildung 4.8: $\Delta R(\tau)$ : (a)Variation $E_{\text {pump }}$ bei $E_{\text {probe }}=1,47 \mathrm{eV}$ und $F=17 \mu \mathrm{Jcm}^{-2}$ (daneben: schematisch: optische Übergänge von Pump- und Probepulsen); (b) Variation der Anregungsfluenz $F$ bei $E_{\text {probe }}=1,47 \mathrm{eV} ; E_{\text {pump }}=1,49 \mathrm{eV}$ (daneben: Maxima des $\Delta R / R_{0}$ Signals als Funktion der Fluenz) 
In der Abbildung 4.8(a) erkennt man für $E_{\text {pump }}=1,55 \mathrm{eV}$ zu frühen Zeiten ein negatives $\Delta R$ Signal und ein positives Signal für $\tau>400 \mathrm{fs}$. Dieser signifikante Verlauf ist mit einer vom Pumppuls generierten Ladungsträgerverteilungsfunktion, die ihren Schwerpunkt energetisch oberhalb der vom Probepuls getesteten Übergänge besitzt, zu erklären. Aufgrund der Thermalisierung und Abkühlung der Ladungsträger (siehe Kap. 2) wird der energetische Schwerpunkt der Ladungsträgerverteilungsfunktion unterhalb der getesteten Übergänge abgesenkt, so daß zu späteren Zeiten $\tau$ ein positives $\Delta R$ Signal gemessen wird. Unter den hier gewählten Anregungsbedingungen findet also die Thermalisierung und Abkühlung der Ladungsträger auf einer subpikosekunden Zeitskala statt.

Der negative Peak des $\Delta R$ Signales nimmt mit abnehmender Pumpphotonenenergie ab, bis er bei $E_{\text {pump }}=1,49 \mathrm{eV}$ nicht mehr beobachtet wird. Da auch hier $E_{\text {pump }}>E_{\text {probe }}$, deutet das Nichtvorhandensein eins negativen $\Delta R$ Signales auf eine Thermalisierung und Abkühlung der Ladungsträger innerhalb der Pulsdauer von $\tau_{p u l s} \approx 100 \mathrm{fs}$ hin.

Die lineare Abhängigkeit des $\Delta R$ Signals von der Fluenz für Fluenzen $F<30 \mu \mathrm{Jcm}^{-2}$ wird in Abbildung 4.8(b) gezeigt.

\subsubsection{Vergleich von $\Delta R(\tau)$ und $\Delta T(\tau)$ in cis und trans Geometrie}

Um die Anwendbarkeit der in Kap. 3 vorgestellten Flugzeitmeßmethode zu demonstrieren, sind $\Delta R(\tau)$ und $\Delta T(\tau)$ in cis und trans Geometrie direkt nacheinander bei einer Probendicke von $d=2 \mu \mathrm{m}$ bei Raumtemperatur gemessen worden. Die Ladungsträger wurden mit Pumpphotonenenergie von $E_{\text {pump }}=E_{G}+470 \mathrm{meV}$ (OPA Signal Pulse) generiert. Die Absorptionslänge bei dieser Photonenenergie beträgt $1 / \alpha=0,3 \mu \mathrm{m}$. In diesem Experiment wurde eine Probephotonenenergie von $E_{\text {probe }}=E_{G}+56 \mathrm{meV}(\operatorname{Reg} \mathrm{A}$ Pulse) gewählt, so daß nur hh-c und lh-c Übergänge (siehe Kap. 3.3 getestet werden.

Das $\Delta R_{\text {cis }}(\tau)$ Signal (siehe Abb. 4.9(a)) erreicht nach einem negativen Peak (Diskussion siehe Kap. 4.4.1) bei $\tau=3$ ps ein Maximum mit einem anschließenden Abfall auf einer 100ps Zeitskala. Im Vergleich zu $\Delta R_{\text {cis }}(\tau)$ ist der zeitliche Verlauf von $\Delta R_{\text {trans }}(\tau)$ signifikant verschieden. Nach einem instantanen Anstieg bei $\tau=0$ kann ein langsamer Anstieg mit einem Maximum bei ca. 150ps eindeutig identifiziert werden (siehe Abb. $4.9(\mathrm{~b}))$. Nach 150ps fangen die $\Delta R_{\text {cis }}(\tau)$ und $\Delta R_{\text {trans }}(\tau)$ Kurven an sich anzunähern, um nach 300ps innerhalb des experimentellen Fehlers identisch zu verlaufen.

Das $\Delta R_{\text {trans }}(\tau)$ Signal kann prinzipiell in zwei Beiträge unterteilt werden.

- Der erste wird von Ladungsträgern verursacht, welche schon innerhalb des Detektionsvolumen angeregt werden. Dieser Beitrag sollte eine dem $\Delta R_{c i s}(\tau)$ Signal vergleichbare Zeitentwicklung besitzen und führt zu dem instantanen Anstieg des $\Delta R_{\text {trans }}(\tau)$ Signales.

- Der zweite Beitrag wird von Ladungsträgern verursacht, welche ursprünglich nicht innerhalb des Detektionsvolumens angeregt wurden, dieses aber später erreichen. 
Das Maximum bei $\tau=150$ ps wird unzweifelhaft von diesem Ladungsträgertransport verursacht. Der identische Verlauf der $\Delta R_{\text {cis }}(\tau)$ und $\Delta R_{\text {trans }}(\tau)$ Kurven nach 300ps kann mit einer örtlich homogenen Ladungsträgerverteilung erklärt werden, welche sich zu diesem Zeitpunkt eingestellt hat.

Wie erwartet sind die $\Delta T_{\text {cis }}(\tau)$ und $\Delta T_{\text {trans }}(\tau)$ Kurven identisch für alle Verzögerungszeiten $\tau$ (siehe Abb. 4.9(c)), da in der $\Delta T$ Messung eine räumliche Mittelung über die Probendicke stattfindet.

Die Messung von $\Delta R(\tau)$ und $\Delta T(\tau)$ in cis und trans Geometrie ermöglicht eine direkte Untersuchung des Ladungsträgertransportes in die Tiefe der Probe.
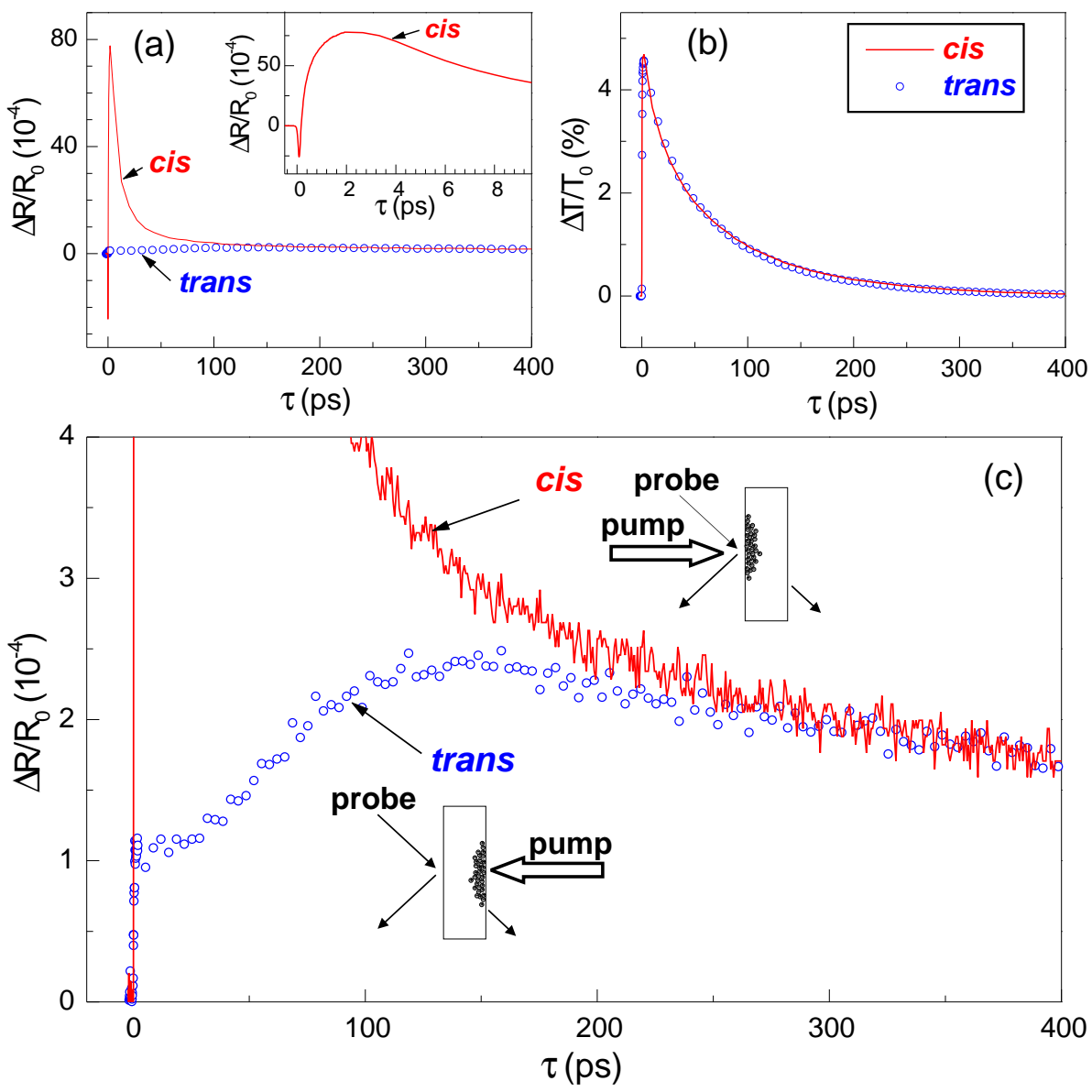

Abbildung 4.9: Vergleich von $\Delta R(\tau)$ und $\Delta T(\tau)$ in cis und trans Geometrie: $E_{\text {pump }}=$ $E_{G}+470 \mathrm{meV} ; E_{\text {probe }}=E_{G}+56 \mathrm{meV} ; F=160 \mu \mathrm{Jcm}^{-2}$. (a) $\Delta R_{\text {cis }}(\tau)$ (Linie) und $\Delta R_{\text {trans }}(\tau)$ (offene Kreise) ,Inset $\Delta R_{\text {cis }}(\tau)$ für kleine Verzögerungszeiten $\tau$, (b) $\Delta T_{\text {cis }}(\tau)$ (Linie) and $\Delta T_{\text {trans }}(\tau)$ (offene Kreise), (c) $\Delta R_{\text {cis }}(\tau)$ und $\Delta R_{\text {trans }}(\tau)$ mit 20 facher Vergrößerung. 


\subsubsection{Differentielle Reflexion am soh-c Übergang}

Im Abschnitt 3.3 wurde die Möglichkeit der Erhöhung der örtlichen Auflösung durch die Wahl der Probephotonenenergie in der Nähe des Übergangs vom Spin-Bahn-abgespaltenen Lochbandes (soh) zum Leitungsband (c) diskutiert. Die Anwendbarkeit der Methode des Testens des soh-c Übergangs auf die Messung der differentiellen Reflexion $\Delta R$ muß gezeigt werden. Insbesondere muß die Frage nach der Größe der Beiträge, der gleichzeitig zum soh-c Übergang mit getesteten hh-c und lh-c Übergänge, beantwortet werden.

Hierfür wurden $\Delta R_{\text {cis }}(\tau)$ Messungen durchgeführt, bei denen die Probephotonenenergie $E_{\text {probe }}$ um die Energie des soh-c Übergangs variiert wurde. Als Probepulse wurden in diesem Energiebereich (GaAs 300K: $E_{G}+\Delta_{s o h}=1,93 \mathrm{eV} \Leftrightarrow \lambda=720 \mathrm{~nm}$ ) spektral mit Hilfe des Prismenpulsformers (siehe Kap. 4.1.1) gefilterte Pulse des Weißlichtkontinuums (siehe Kap. 4.1) eingesetzt. Die spektrale Auflösung ist durch die spektrale Breite der Probepulse von $\sim 16 \mathrm{meV}$ begrenzt. Die Ladungsträger wurden in der Nähe der Bandkante mit $E_{\text {pump }}=E_{G}+100 \mathrm{meV}$ mit Hilfe von RegA Pulsen generiert. In der Abbildung 4.10 sind drei repräsentative Meßkurven zusammen mit einer schematischen Darstellung der Übergänge von Pump und Probepulsen dargestellt.

Bei einer Probephotonenenergie von $52 \mathrm{meV}$ oberhalb des soh-c Übergangs wird ein relativ großes positives $\Delta R_{\text {cis }}(\tau)$ Signal gemessen (siehe Abb. 4.10(a)). In diesem Fall werden die Elektronen energetisch unterhalb des getesteten soh-c Übergangs generiert. Das gemessene positive $\Delta R_{\text {cis }}(\tau)$ stimmt mit den Betrachtungen aus Kap. 3.1.1 überein.

Bei einer Probephotonenenergie von $E_{\text {probe }}=E_{g}+\Delta_{\text {soh }}+10 \mathrm{meV}$, wo die Elektronen energetisch oberhalb des getesteten soh-c Übergangs generiert werden, ist wieder der signifikante Verlauf mit dem anfänglichen negativen Peak mit einem anschließenden positiven $\Delta R$ Signal zu beobachten. Wie in Kap. 4.4.1 ausführlich dargelegt, wird dieser Verlauf von der energetischen Relaxation der Ladungsträger (hier nur der Elektronen) verursacht.

Die wichtigste Schlußfolgerung, daß nämlich die immer mit getesteten Übergänge hh-c und lh-c hier keinen meßbaren Beitrag zu dem $\Delta R$ Signal leisten, kann man aus der Meßkurve in Abbildung 4.10(c) ziehen. Dort wird bei einer Probephotonenenergie energetisch knapp unterhalb des soh-c Übergangs kein zeitabhängiges $\Delta R_{\text {cis }}(\tau)$ Signal gefunden. Dieser experimentelle Befund hat zwei Ursachen. Erstens ist die Anzahl der Ladungsträger bei der Energie der hh-c und lh-c Übergänge gering, und zweitens verursachen diese Ladungsträger aufgrund einer dort vorhandenen höheren Zustandsdichte eine geringere Phasenraumfüllung und damit eine geringere Änderung der optischen Eigenschaften. 


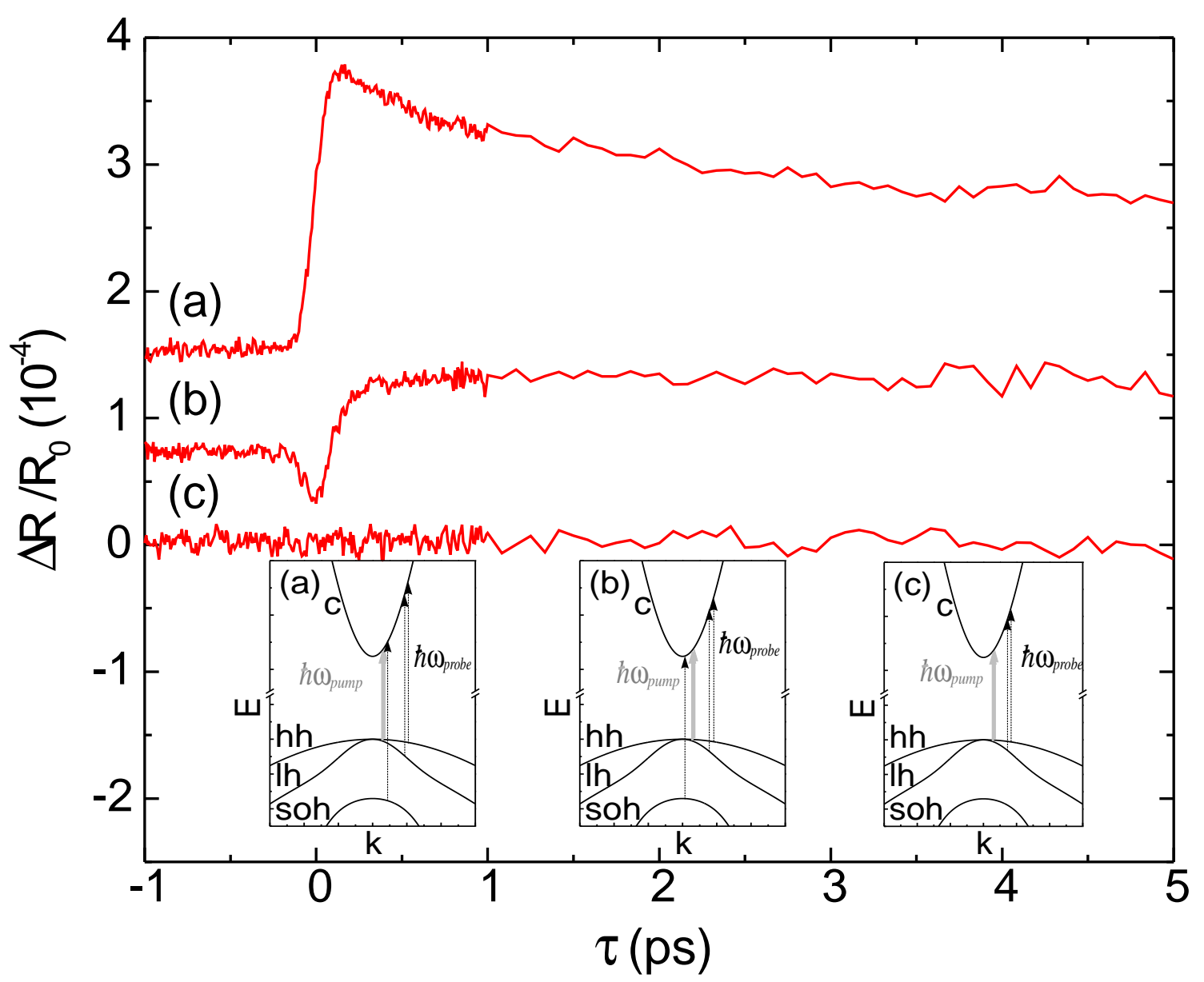

Abbildung 4.10: $\Delta R_{\text {cis }}$ bei Variation von $E_{\text {probe }}$ um den soh-c Übergang: (a) $E_{\text {probe }}=$ $E_{g}+\Delta_{\text {soh }}+51 \mathrm{meV}$, (b) $E_{\text {probe }}=E_{g}+\Delta_{\text {soh }}+10 \mathrm{meV}$, (c) $E_{\text {probe }}=E_{g}+\Delta_{\text {soh }}-90 \mathrm{meV}$. In den Insets sind eine schematische Bandstruktur zusammen mit den optischen Übergängen der Pumppulse (durchgezogene Pfeile) und der Probepulse (gestrichelte Pfeile) dargestellt.

Die hier vorgestellten Meßergebnisse führen zu der Schlußfolgerung, daß $\Delta R$ Messungen am soh-c Übergang sensitiv auf vom Pumppuls generierte Ladungsträgerverteilungen sind und somit eine örtliche Auflösung der Flugzeitmeßmethode von $\frac{1}{2}\left(1 / \alpha_{\text {probe }}\right) \sim 150 \mathrm{~nm}$ erzielt werden kann.

Für die Flugzeituntersuchungen in Kap. 5 wurde eine Probephotonenenergie von $E_{\text {probe }}=E_{G}+\Delta_{\text {soh }}+54 \mathrm{meV}$ gewählt, bei der das größte $\Delta R$ Signal vom soh-c Übergang erzielt wird. 


\section{Kapitel 5}

\section{EHP-Expansion bei $T_{L}=300 \mathrm{~K}$}

Nachdem in den vorhergegangenen Kapiteln sowohl die Idee der Flugzeitmeßmethode als auch die konkrete experimentelle Realisierung vorgestellt wurde, soll in diesem Kapitel mit dieser Methode die Elektron-Loch-Plasma-Expansion in GaAs bei Raumtemperatur $\left(T_{L}=300 \mathrm{~K}\right)$ untersucht werden. Zuerst wird die Messung von $\Delta R(\tau)$ und $\Delta T(\tau)$ in cis Geometrie (Anregung und Detektion auf der Vorderseite der Probe) bei Variation der Pumpfluenz diskutiert. Diese Messung gibt einen ersten Hinweis auf eine schnelle Plasmaexpansion mit zunehmender Pumpfluenz. Diese EHP-Expansion wird dann mit Hilfe von Flugzeitmessungen detailliert spektroskopiert. Insbesondere werden die Zeitabhängigkeit der Expansionsgeschwindigkeit und die Abhängigkeit der Expansion von der Pumpphotonenenergie und der Pumpfluenz untersucht.

Im Anschluß daran wird im Rahmen eines hydrodynamischen Thermodiffusionsmodells der Frage nach der treibenden Kraft der untersuchten EHP-Expansion nachgegangen. Im Rahmen dieses Modells werden Modellrechnungen durchgeführt, die dann mit den experimentellen Ergebnissen verglichen werden.

\subsection{Experimentelle Untersuchungen}

\subsubsection{Erste Hinweise einer EHP-Expansion}

Wie bereits oben erwähnt, wird als erstes die Messung von $\Delta R_{\text {cis }}(\tau)$ und $\Delta T_{\text {cis }}(\tau)$ bei einer Variation der Anregungsfluenz vorgestellt. Im Gegensatz zu den Laufzeituntersuchungen der folgende Abschnitte, bei denen immer der soh-c Übergang getestet wird, ist hier eine Probephotonenenergie von $E_{\text {Probe }}=E_{G}+60 \mathrm{meV}$ (RegA-Pulse) eingesetzt worden. Die Anregung erfolgte bei einer Energie von $E_{\text {pump }}=E_{G}+670 \mathrm{meV}$ (OPA Signal Pulse) mit einer Absorptionslänge von $1 / \alpha_{\text {pump }}=0,2 \mu \mathrm{m}$.

In der Abbildung 5.1 sind die differentiellen Änderungen der Reflexion und Transmission normiert aufgetragen. Man erkennt, daß die $\Delta T_{\text {cis }}(\tau)$ Kurven für alle verwendeten Fluenzen $F$ im Rahmen des experimentelle Fehler identisch sind. Die $\Delta T$ Signale erreichen nach einem raschen Anstieg bei $\tau \sim 6 \mathrm{ps}$ ein Maximum, dem ein Abfall auf einer 100ps 

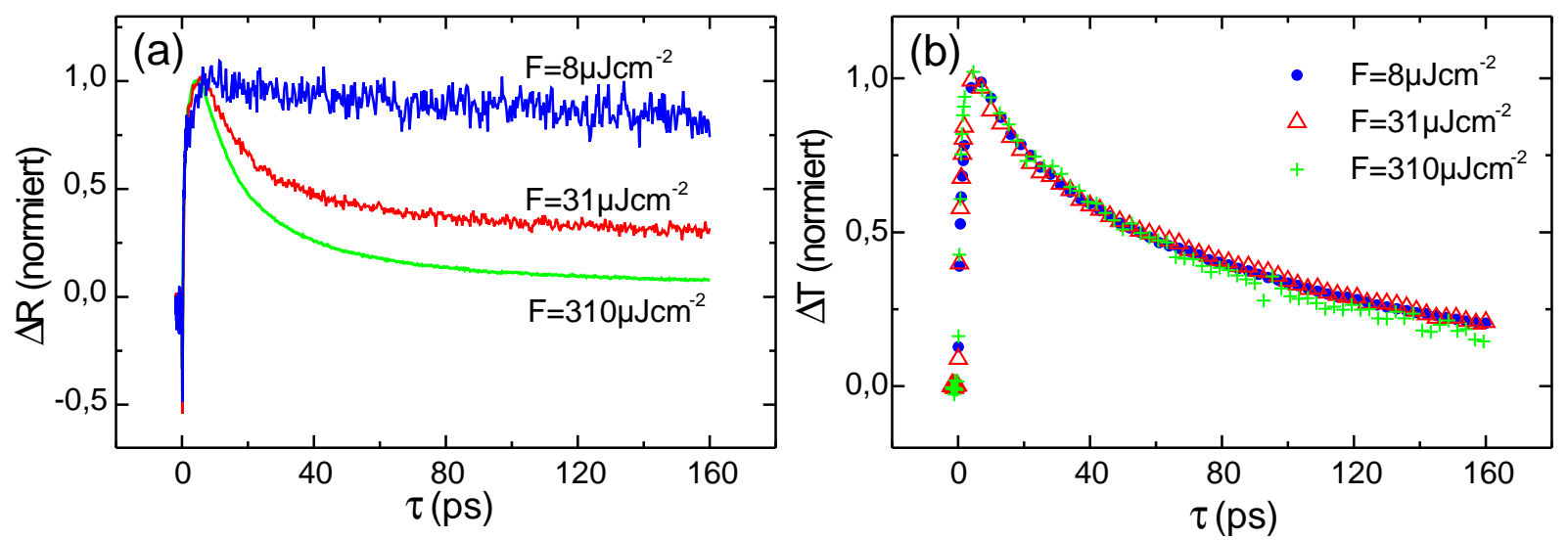

Abbildung 5.1: (a) $\Delta R_{\text {cis }}(\tau)$ und (b) $\Delta T_{\text {cis }}(\tau)$ bei Variation der Fluenz $F\left(E_{\text {Probe }}=\right.$ $\left.E_{G}+60 \mathrm{meV}, E_{\text {pump }}=E_{G}+670 \mathrm{meV}\right)$

Zeitskala folgt. Der Verlauf der $\Delta T$ Signale kann mit der schnellen $(\sim$ ps $)$ energetischen Relaxation der mit einer hohen kinetischen Energie angeregten Ladungsträger erklärt werden, die dann auf einer Zeitskala von einigen 100 ps bis ns rekombinieren.

Im Gegensatz zu den $\Delta T$ Signalen ist der Verlauf der $\Delta R_{c i s}$ Signale für die drei Fluenzen deutlich verschieden (siehe Abb. 5.1). Während der anfängliche Verlauf mit dem negativen Peak und dem raschen Anstieg (Diskussion des Verlaufs siehe Kap. 4.4.1) noch vergleichbar für alle drei Fluenzen ist, wird ein deutlich schnellerer Abfall der Signale mit zunehmender Fluenz beobachtet.

Der identische Verlauf der $\Delta T$ Signale läßt darauf schließen, daß die energetische Relaxation der Ladungsträger innerhalb des Bereiches, der hier variiert wurde, unabhängig von der Pumpfluenz ist. Somit kann die Abhängigkeit der Verläufe der $\Delta R_{\text {cis }}$ Signale von der Pumpfluenz bei identischem Verlauf der $\Delta T_{\text {cis }}$ Signale nur mit der unterschiedlichen örtlichen Sensitivität von $\Delta R$ und $\Delta T$ interpretiert werden. Wie in Kap. 3.2 dargelegt, ist die $\Delta R$ Messung nur sensitiv auf oberflächennahe Ladungsträger. Die differentielle Transmission ist demgegenüber sensitiv auf alle Ladungsträger innerhalb der Probendicke. Ein Transport des oberflächennah angeregten Elektron-Loch-Plasmas in die Tiefe der Probe verursacht also keine Änderung von $\Delta T_{c i s}$, aber eine Abnahme $\Delta R_{\text {cis }}$.

Somit lassen sich die experimentellen Ergebnisse von $\Delta R_{\text {cis }}(\tau)$ und $\Delta T_{\text {cis }}(\tau)$ bei Variation der Fluenz mit einer von der Anregungsfluenz abhängigen Ausbreitung des Elektron-Loch-Plasmas in die Tiefe der Probe interpretieren.

Aussagen über die Ausbreitungsgeschwindigkeit des EHP oder sogar über die Zeitabhängigkeit dieser Geschwindigkeit lassen sich aus diesen Messungen jedoch nur sehr indirekt über entsprechende Modellvorstellungen gewinnen. Somit sollen diese Messungen in dieser Arbeit nur als ein erster Hinweis auf eine schnelle Ausbreitung des EHP dienen. Die EHP-Expansion in GaAs soll nun detailliert in den nächsten Abschnitten mit Hilfe der in Kap. 3 vorgestellten Flugzeitmeßmethode untersucht werden. 
Viele der in der Literatur vorgestellten Untersuchungen zur EHP-Expansion wurden jedoch anhand von Messungen, wie der zeitaufgelösten Lumineszenz [Pol96, Pol99, Nak92] und zeitaufgelöste Ramanstreuung [Tse89], durchgeführt, bei denen die Anregung und Detektion der Ladungsträger lediglich auf der selben Probenseite erfolgte.

\subsubsection{Flugzeitmessung: Variation der Probendicke}

Als Ausgangspunkt für die Untersuchung der EHP-Expansion in GaAs sollen Flugzeitmessungen über unterschiedliche Probendicken $d$ bei einer Anregungsfluenz von $F=$ $800 \mu \mathrm{Jcm}^{-2}$ vorgestellt werden. Die Ladungsträger wurden hier mit einer Pumpphotonenenergie von $E_{\text {pump }}=E_{G}+1,0 \mathrm{eV}$ (OPA Signal Pulse) bei einer resultierenden Absorptionslänge von $1 / \alpha=0,11 \mu \mathrm{m}$ angeregt. Alle Flugzeituntersuchungen bei Raumtemperatur wurden mit einer Probephotonenenergie von $E_{\text {probe }}=E_{G}+\Delta_{\text {soh }}+54 \mathrm{meV}$ durchgeführt, bei denen der soh-c Übergang getestet wird (siehe Kap. 4.4.3).

Wie in Kap. 3.2 dargelegt, kann man prinzipiell aus der $\Delta R_{\text {trans }}(\tau)$ Messung die Ankunftszeit der Ladungsträger, die ursprünglich auf der gegenüberliegenden Probenseite angeregt wurden, bestimmen. Nun ist aber eine einfache Interpretation von Flugzeitmessungen nur möglich, wenn die Antwortzeit des Detektors kürzer als die Zeitdauer des zu untersuchenden Transportes ist. In der hier verwendeten Flugzeitmeßmethode dient das $\Delta R_{\text {trans }}(\tau)$ Signal als Detektor für die Ladungsträger. Aufgrund der energetischen Relaxation der Ladungsträger hat das $\Delta R$ Signal selbst bei einer (hypothetisch) räumlich homogenen Probenanregung eine endliche Anstiegszeit. Als Maß für diese Zeit kann in guter Näherung die Anstiegszeit des $\Delta R_{\text {cis }}$ Signals verwendet werden. Damit man $\Delta R_{\text {trans }}(\tau)$ direkt als Maß für die Ankunftszeit der Ladungsträger benutzen kann, muß die Anstiegszeit des $\Delta R_{\text {cis }}(\tau)$ Signals kürzer sein als die Anstiegszeit der $\Delta R_{\text {trans }}(\tau)$ Signale. Um zu zeigen, daß dieses in den hier durchgeführten Messungen der Fall ist, sind im Inset von Abbildung 5.2 $\Delta R_{\text {cis }}(\tau)$ und $\Delta R_{\text {trans }}(\tau)$, gemessen an der dünnsten Probenstelle $d=0,8 \mu \mathrm{m}$, zusammen aufgetragen. Man erkennt, daß das $\Delta R_{\text {cis }}(\tau)$ Signal schon wesentlich früher $(\sim 6 \mathrm{ps})$ das Maximum erreicht, als das bei $\Delta R_{\text {trans }}(\tau)$ der Fall ist.

Mit der Variation der Probendicke $d$ ändert sich das $\Delta R_{\text {trans }}(\tau)$ Signal drastisch (siehe Abb. 5.2). In den $\Delta R_{\text {trans }}(\tau)$ Signalen für Probendicken $d<1,4 \mu \mathrm{m}$ ist ein instantaner Anstieg bei $\tau=0 \mathrm{zu}$ erkennen, der auf Ladungsträger zurückgeführt werden kann, die schon im Detektionsvolumen von $\Delta R_{\text {trans }}$ angeregt wurden (siehe Kap. 4.4.2). Die Abnahme der Intensität des instantanen Anstiegs mit zunehmender Probendicke $d$ unterstützt diese Interpretation. Für Probendicken $d \geq 1,4 \mu \mathrm{m}$ ist kein instantanes Signal bei $\tau=0 \mathrm{zu}$ erkennen und das $\Delta R_{\text {trans }}(\tau)$ Signal bleibt konstant Null bis zu einem relativ schnellen Anstieg bei großen Verzögerungszeiten. Der Zeitpunkt des Anstiegs ist von der Probendicke $d$ abhängig und ist ein Maß für die Zeit, die die Ladungsträger brauchen, um über die Strecke $d$ zu gelangen. Die relativ kurze Anstiegszeit ist bemerkenswert, da sie darauf schließen läßt, daß sich eine relativ steile Ladungsträgerfront durch die Probe bewegt (Diskussion siehe Kap. 5.3.1). 


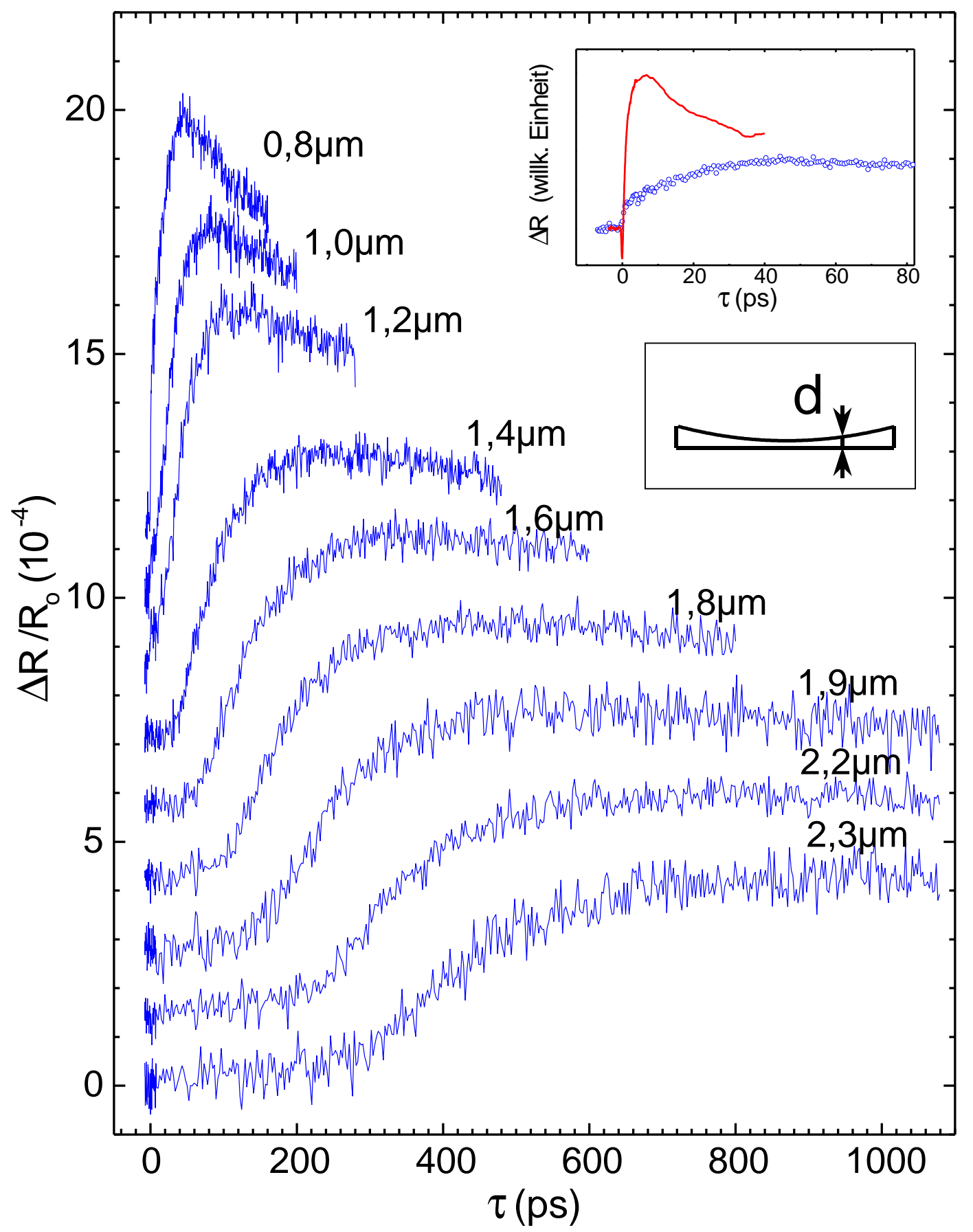

Abbildung 5.2: $\Delta R_{\text {trans }}$ bei Variation der Probendicke $d$. Die Kurven für unterschiedliche Probendicken $d$ wurden hier mit einem konstanten Offset versehen. $\left(E_{\text {probe }}=E_{g}+\Delta_{\text {soh }}+\right.$ $\left.54 \mathrm{meV}, E_{\text {pump }}=2,44 \mathrm{eV}, F=800 \mu \mathrm{Jcm}^{-2}\right)$ Im Inset ist $\Delta R_{\text {cis }}(\tau)$ (durchgezogenen Linie) zusammen mit $\Delta R_{\text {trans }}(\tau)$ bei $d=0,8 \mu \mathrm{m}$ (offene Kreise) aufgetragen. Weiterhin ist eine schematische Probengeometrie mit der von der lateralen Position abhängigen Probendicke $d$ dargestellt. 
Um nun die Transportgrößen wie die Ausbreitungsgeschwindigkeit aus den $\Delta R_{\text {trans }}(\tau)$ Kurven zu extrahieren, muß die Ankunftszeit der Ladungsträger bestimmt werden. Prinzipiell sind mehrere Definitionen einer solchen Ankunftszeit möglich. Als Maß dafür könnte man die Verzögerungszeit $\tau$ bei dem Maximum des $\Delta R_{\text {trans }}(\tau)$ Signals oder auch den Einsetzpunkt des Anstiegs wählen. Da ein Maximum in $\Delta R_{\text {trans }}(\tau)$ nur für Probendicken $d<1,8 \mu \mathrm{m}$ ausgeprägt ist und auch die Bestimmung des Einsetzpunktes nur schwierig möglich ist, wird in dieser Arbeit eine andere Definition verwendet. Als Maß für die Ankunftszeit der Ladungsträger wird die Verzögerungszeit $\tau_{H M}$ bestimmt, bei der das $\Delta R_{\text {trans }}(\tau)$ Signal die Hälfte des jeweils maximalen Signals erreicht hat. Diese Definition hat den Vorteil, daß sich $\tau_{H M}$ eindeutig aus den $\Delta R_{\text {trans }}(\tau)$ Kurven bestimmen läßt und sich somit auch für einen Vergleich mit Modellsimulationen eignet.

Die Abhängigkeit der so bestimmten Ankunftszeit $\tau_{H M}$ von der Probendicke $d$ ist in der Abbildung 5.3 in Form einer Laufzeitkurve (zurückgelegte Strecke $d$ als Funktion der Zeit $\left.\tau_{H M}\right)$ auf einer linearen und einer doppellogarithmischen (Inset) Skala dargestellt.

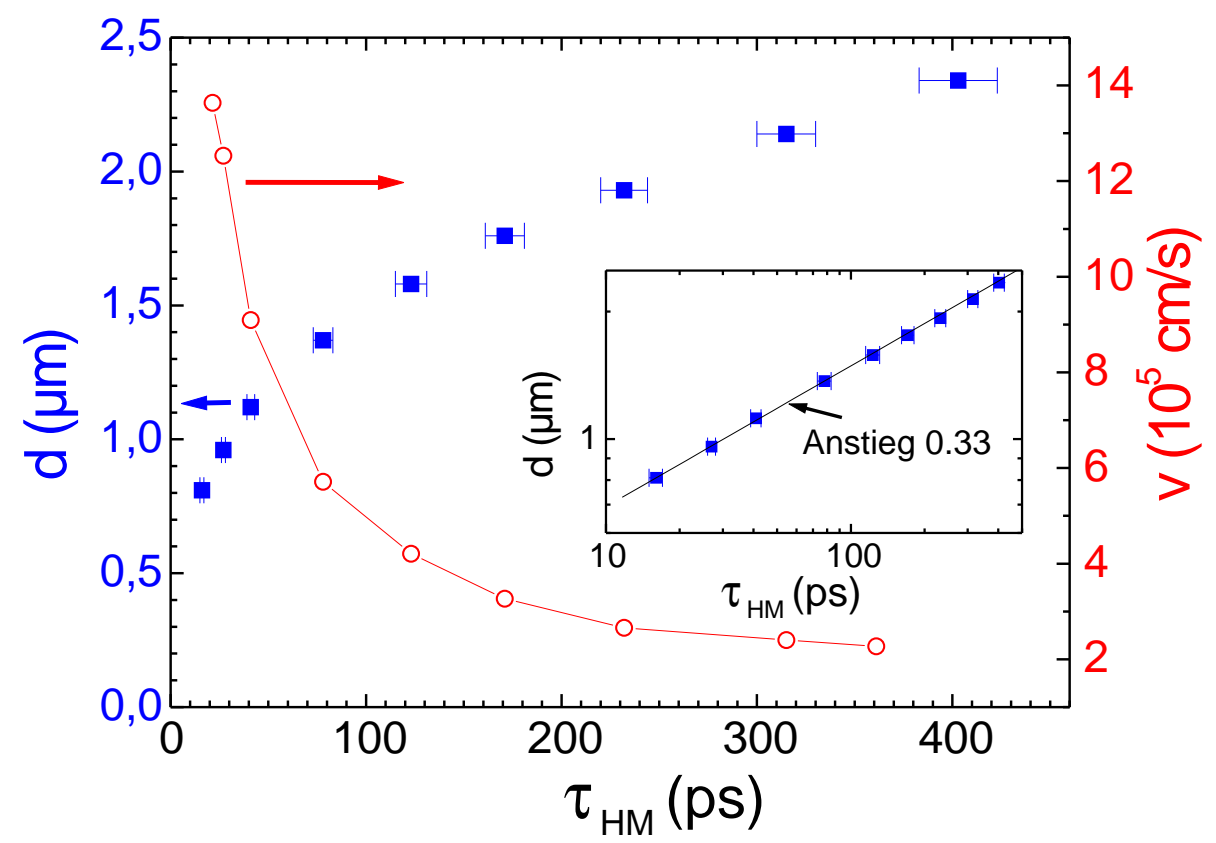

Abbildung 5.3: Laufzeitkurven (Bestimmt aus den $\Delta R_{\text {trans }}(\tau)$ Kurven aus Abb. 5.2): Probendicke $d$ (gefüllte Vierecke + linke Achse) und die daraus resultierenden Geschwindigkeiten (offene Kreise + rechte Achse) sind als Funktion von $\tau_{H M}$ aufgetragen. Im Inset ist die Probendicke $d$ gegen die Ankunftszeit $\tau_{H M}$ in einer doppellogarithmischen Skala aufgetragen.

Aus der Abhängigkeit $d=d\left(\tau_{H M}\right)$ läßt sich nun einfach per Differentiation die zeitabhängige Ausbreitungsgeschwindigkeit $v=v(\tau)$ ermitteln, welche auch in Abbildung 5.3 dargestellt ist. 
Man erkennt, daß sich das Elektron-Loch-Plasma mit einer anfänglich hohen Geschwindigkeit von $v=14 \times 10^{5} \mathrm{~cm} / \mathrm{s}$ bei $\tau=20 \mathrm{ps}$ ausbreitet. Diese anfängliche Geschwindigkeit ist somit signifikant größer als die Schallgeschwindigkeit in GaAs von $v_{\text {Schall }}^{\text {GaAs }} \approx$ $5 \times 10^{5} \mathrm{~cm} / \mathrm{s}$. Die Ausbreitungsgeschwindigkeit nimmt mit zunehmender Verzögerungszeit auf $v=2 \times 10^{5} \mathrm{~cm} / \mathrm{s}$ bei $\tau \approx 400 \mathrm{ps}$ ab.

In der doppellogarithmischen Auftragung von $d=d\left(\tau_{H M}\right)$ liegen alle Datenpunkte innerhalb des experimentellen Fehlers auf einer Geraden mit der Steigung $p=0,33$, so daß die Korrelation zwischen $\tau_{H M}$ und der Probendicke $d$ mit der empirischen Relation

$$
d \propto \tau_{H M}^{p} ; p=0,33
$$

beschrieben werden kann.

Im Vergleich dazu würde ein einfacher diffusiver Ladungstransport mit einer konstanten Diffusivität $D$ zu einem Anstieg von $p=0,5(x \propto \sqrt{t})$ führen. Die hier mit Hilfe der Flugzeitmethode auf dieser Zeit- und Längenskala zum ersten mal direkt gemessene Zeitabhängigkeit der Ausbreitungsgeschwindigkeit kann nun mit theoretischen Überlegungen zur EHP-Expansion verglichen werden. Eine einfache Theorie, die die gemessene Zeitabhängigkeit beschreibt, wird in Abschnitt 5.2 vorgestellt.

\subsubsection{Flugzeitmessung: Variation der Pumpphotonenenergie}

Bei einer Anregung von Halbleitern mit einer Photonenenergie $E \gg E_{G}$ werden Ladungsträger bei $\tau=0$ mit hohen kinetischen Energien $E-E_{G}=\Delta E=E_{k i n}^{e}+E_{k i n}^{h}$ angeregt. Die kinetische Energie wird während der energetischen Relaxation über Ladungsträger-Phononen Streuung an das Gitter abgegeben (siehe Kap. 2). Eine interessante Fragestellung ist nun, ob die Geschwindigkeit der EHP-Ausbreitung von der Größe der anfänglichen kinetischen Energien der Ladungsträger abhängig ist. Um dieses zu untersuchen, wurden Laufzeitmessungen mit unterschiedlichen Überschußenergien der Pumppulse $\Delta E=0,63 \mathrm{eV} ; 1,0 \mathrm{eV}$ und $1,65 \mathrm{eV}$ durchgeführt. Bei diesen Messungen wurde eine identische mittlere Laserleistung im Pumpstrahl verwendet. Auch hier wurden wieder jeweils die Ankunftszeit $\tau_{H M}$ bestimmt. In der Abbildung 5.4 ist die Probendicke $d$ gegen die Ankunftszeit $\tau_{H M}$ für die drei Pumpphotonenenergien aufgetragen.

Man erkennt, daß der Zusammenhang zwischen der Probendicke $d$ und $\tau_{H M}$ qualitativ für alle drei Kurven gleich ist. Auf den ersten Blick erstaunlich ist, daß die Ladungsträger, die ursprünglich die geringste kinetische Energie besaßen, die schnellsten zu sein scheinen. Zwei Gründe könnten für dieses Verhalten verantwortlich sein. Der erste ist, daß mit zunehmender Photonenenergie die Absorptionslänge $1 / \alpha_{\text {pump }}$ kleiner wird. Somit werden mit zunehmendem $E_{\text {pump }}$ die Ladungsträger räumlich näher an der Oberfläche generiert und der mittlere Laufweg der Ladungsträger zu der gegenüberliegenden Probenseite wird größer (siehe Abb. 5.5). Zweitens nimmt mit zunehmendem $E_{\text {pump }}$ bei identischer mittlerer Laserleistung die Anzahl der Photonen im Pumppuls und damit auch die der generierten 


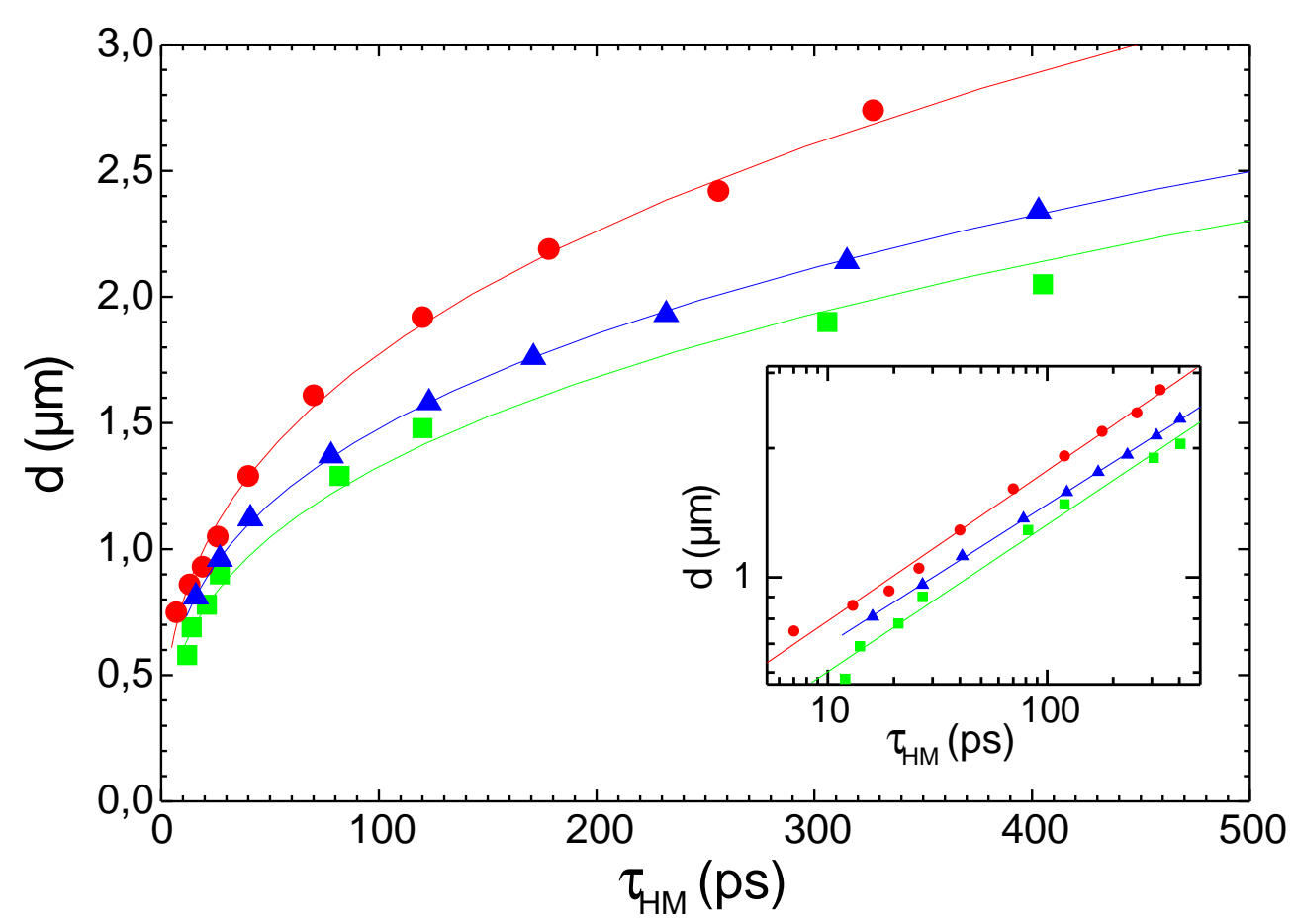

Abbildung 5.4: Laufzeitkurven für unterschiedliche Pumpphotonenenergien $E_{\text {pump }}$ auf einer linearen und doppellogarithmischen Skala (inset): Quadrate: $E_{\text {pump }}=E_{G}+1,65 \mathrm{eV}$; Dreiecke: $E_{\text {pump }}=E_{G}+1 \mathrm{eV}$ und Kreise: $E_{\text {pump }}=E_{G}+0,63 \mathrm{eV}$ mit der selben mittleren Laserleistung entsprechend einer Fluenz von $F \approx 800 \mu \mathrm{Jcm}^{-2}$.

Elektron-Loch Paare ab. Auf die starke Dichteabhängigkeit der EHP-Expansion wird im nächsten Abschnitt eingegangen. Somit haben mit zunehmenden $E_{\text {pump }}$ die angeregten Ladungsträger zwar höhere kinetische Energien, aber es werden weniger Ladungsträger und diese auch noch näher an der Oberfläche angeregt. Das Zusammenspiel dieser Faktoren auf die EHP-Ausbreitung macht eine einfache Interpretation der Messungen schwierig.
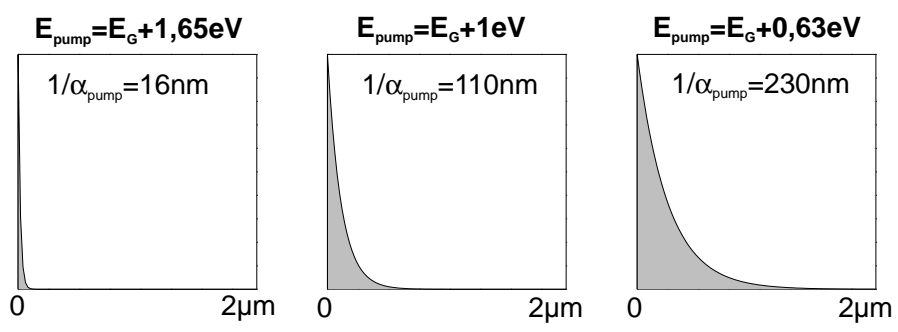

Abbildung 5.5: Schematische Darstellung der Anregungsprofile für die verwendeten Pumpphotonenenergien. 


\subsubsection{Flugzeitmessung: Variation der Pumpfluenz}

Um die Abhängigkeit der Elektron-Loch-Plasma-Ausbreitung von der Dichte der angeregten Ladungsträger zu studieren, wurden zwei Meßreihen durchgeführt. In der ersten wurde $\Delta R_{\text {trans }}(\tau)$ bei einer festen Probendicke $d=1,0 \mu \mathrm{m}$ mit unterschiedlichen mittleren Leistungen im Pumpstrahl gemessen. In der zweiten Meßreihe wurde für jeweils 3 Pumpfluenzen $F \Delta R_{\text {trans }}(\tau)$ für unterschiedlich Probendicken $d$ bestimmt.

Zuerst soll hier die $\Delta R_{\text {trans }}(\tau)$ Messung bei festem $d$ unter Variation von $F$ vorgestellt werden. Die Variation der Laserleistung geschah mit Hilfe eines variablen Graufilters, so daß alle anderen experimentellen Parameter, insbesondere die laterale Position des Pumpstrahlfokus auf der Probe, im Rahmen der Meßgenauigkeit identisch blieben.

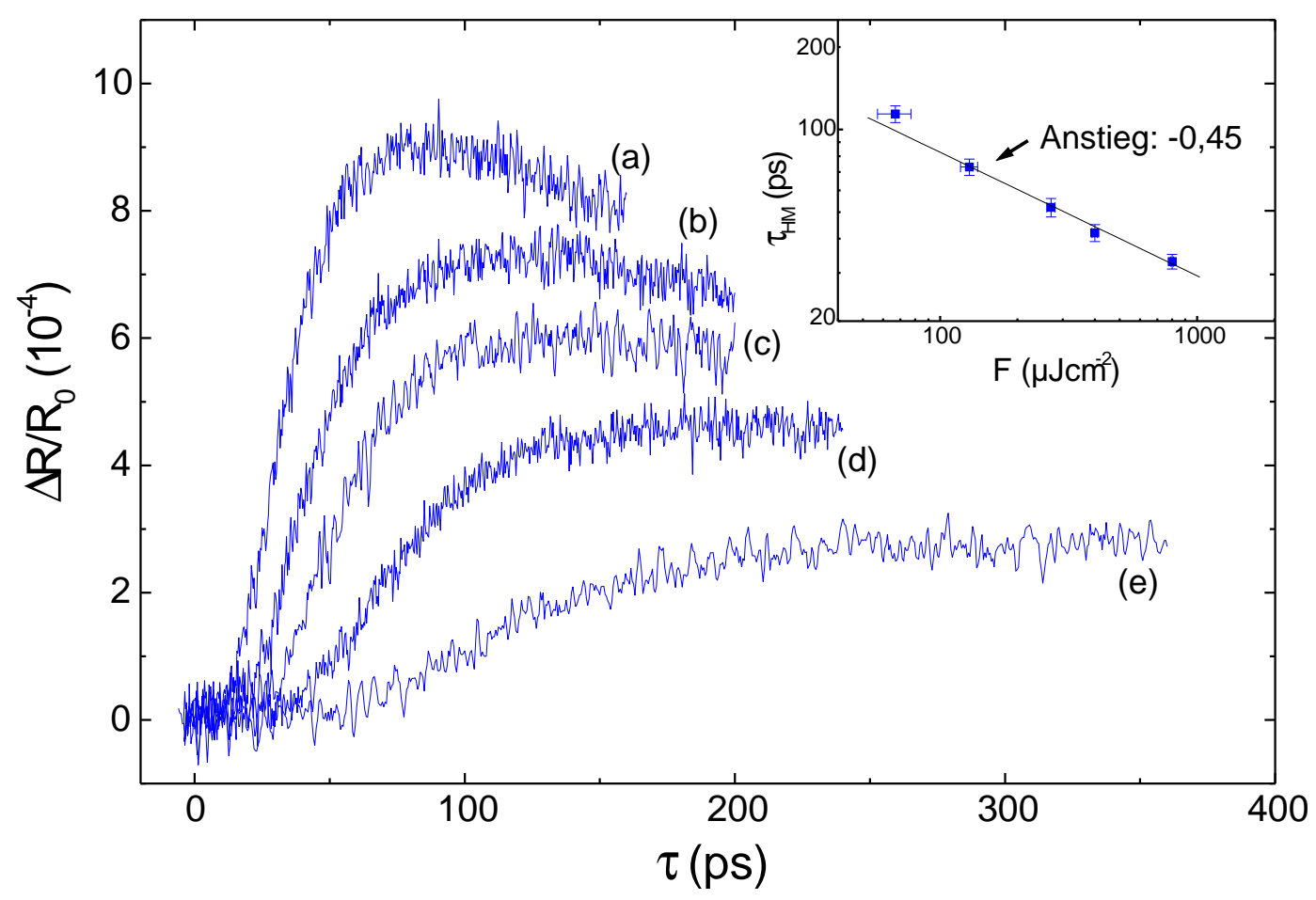

Abbildung 5.6: $\Delta R_{\text {trans }}(\tau)$ : Variation von $F$ bei $d=1,0 \mu \mathrm{m}\left(E_{\text {pump }}=E_{g}+1 \mathrm{eV}\right)$. (a) $F=800 \mu \mathrm{Jcm}^{-2}$, (b) $F=400 \mu \mathrm{Jcm}^{-2}$, (c) $F=270 \mu \mathrm{Jcm}^{-2}$, (d) $F=130 \mu \mathrm{Jcm}^{-2}$ und (e) $F=67 \mu \mathrm{Jcm}^{-2}$. Im Inset ist $\tau_{H M}$ als Funktion von der Fluenz doppellogarithmisch aufgetragen.

Die sehr starke Abhängigkeit der EHP-Expansion von der Fluenz der Pumppulse ist in der Abbildung 5.6 dargestellt. Man erkennt, daß mit zunehmender Fluenz in dem Bereich $67 \mu \mathrm{Jcm}^{-2} \leq F \leq 800 \mu \mathrm{Jcm}^{-2}$ sowohl die Anstiegszeit als auch die Zeit, bei der der Anstieg stattfindet, wesentlich kleiner wird. 
Der Zusammenhang zwischen der Ankunftszeit der Ladungsträger $\tau_{H M}$ und der Pumpfluenz F kann in guter Näherung mit

$$
\tau_{H M} \propto 1 / F^{0,45}
$$

beschrieben werden.

Alle $\Delta R_{\text {trans }}(\tau)$ Meßkurven der zweiten Meßreihe sind in der Abbildung 5.7 zu sehen. $\mathrm{Zu}$ beachten ist, daß die Meßkurven für unterschiedliche Probendicken $d$ mit einer unterschiedlichen Skalierung sowohl der $\tau$-Achse als auch der $\Delta R / R_{0}$-Achse dargestellt sind.

Aus der Abbildung 5.7 erkennt man, daß für alle Probendicken $0,8 \mu \mathrm{m} \leq d \leq 2,3 \mu \mathrm{m}$ mit der Erhöhung der Fluenz ein früherer Anstieg des $\Delta R_{\text {trans }}(\tau)$ Signals gemessen wird. Für Probendicken $d>1,8 \mu \mathrm{m}$ wurde für $F=67 \mu \mathrm{Jcm}^{-2}$ keine Zeitabhängigkeit des $\Delta R_{\text {trans }}(\tau)$ Signals gefunden. Bei den hohen Probendicken sind deshalb nur noch die Meßkurven für die (hohen) Fluenzen aufgetragen, für die ein signifikanter Anstieg noch meßbar war.
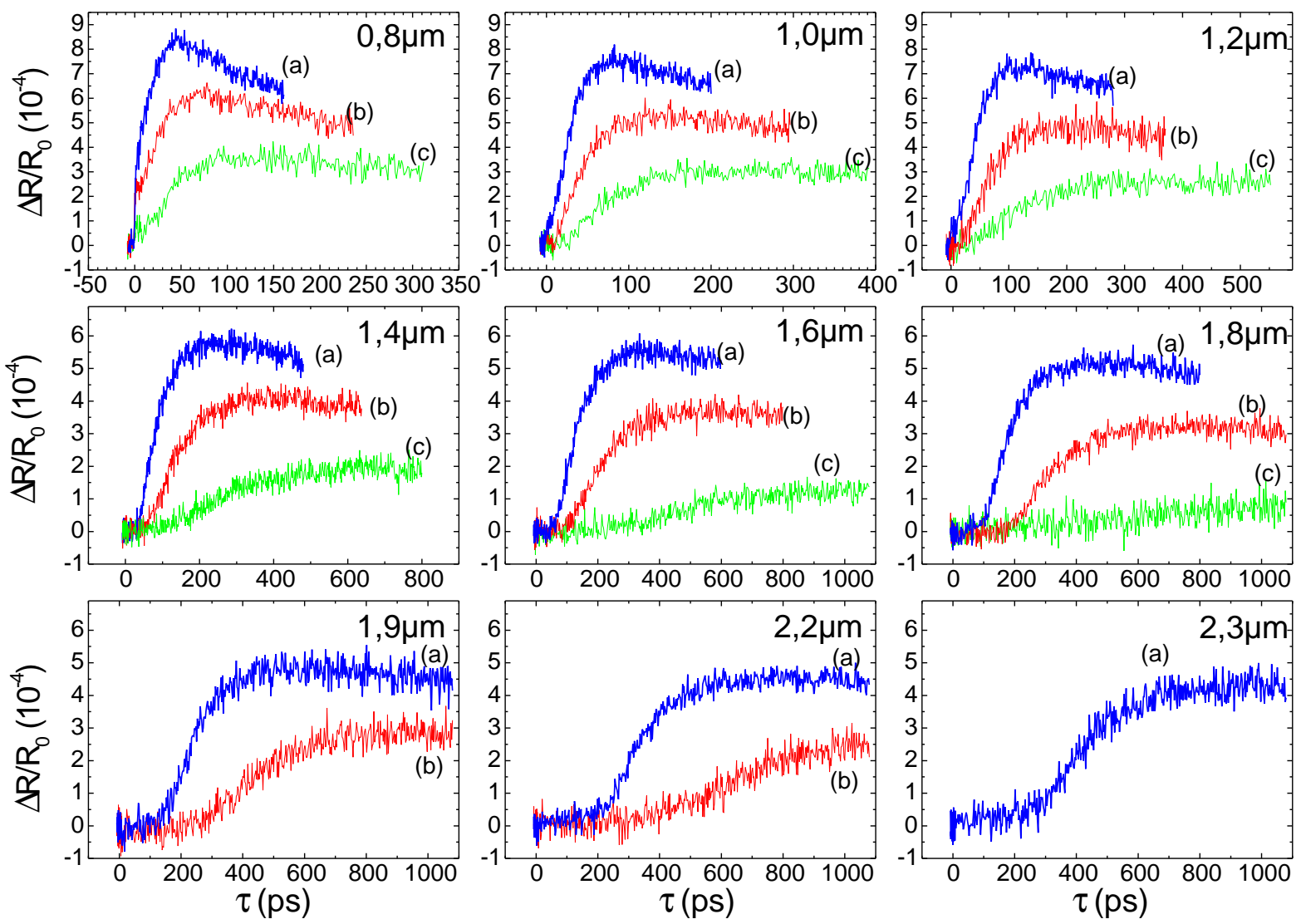

Abbildung 5.7: $\Delta R_{\text {trans }}(\tau)$ : Variation der Probendicke und Pumpfluenz $\left(E_{\text {pump }}=E_{g}+\right.$ $1 \mathrm{eV}$ ) (a) $F=800 \mu \mathrm{Jcm}^{-2}$, (b) $F=270 \mu \mathrm{Jcm}^{-2}$, (c) $F=67 \mu \mathrm{Jcm}^{-2}$. 
Die aus den in Abbildung 5.7 dargestellten Meßkurven bestimmten Ankunftszeiten $\tau_{H M}$ der Ladungsträger sind in der Abbildung 5.8 als Funktion der Probendicke $d$ für die drei unterschiedlichen Fluenzen $F$ linear und doppellogarithmisch aufgetragen.

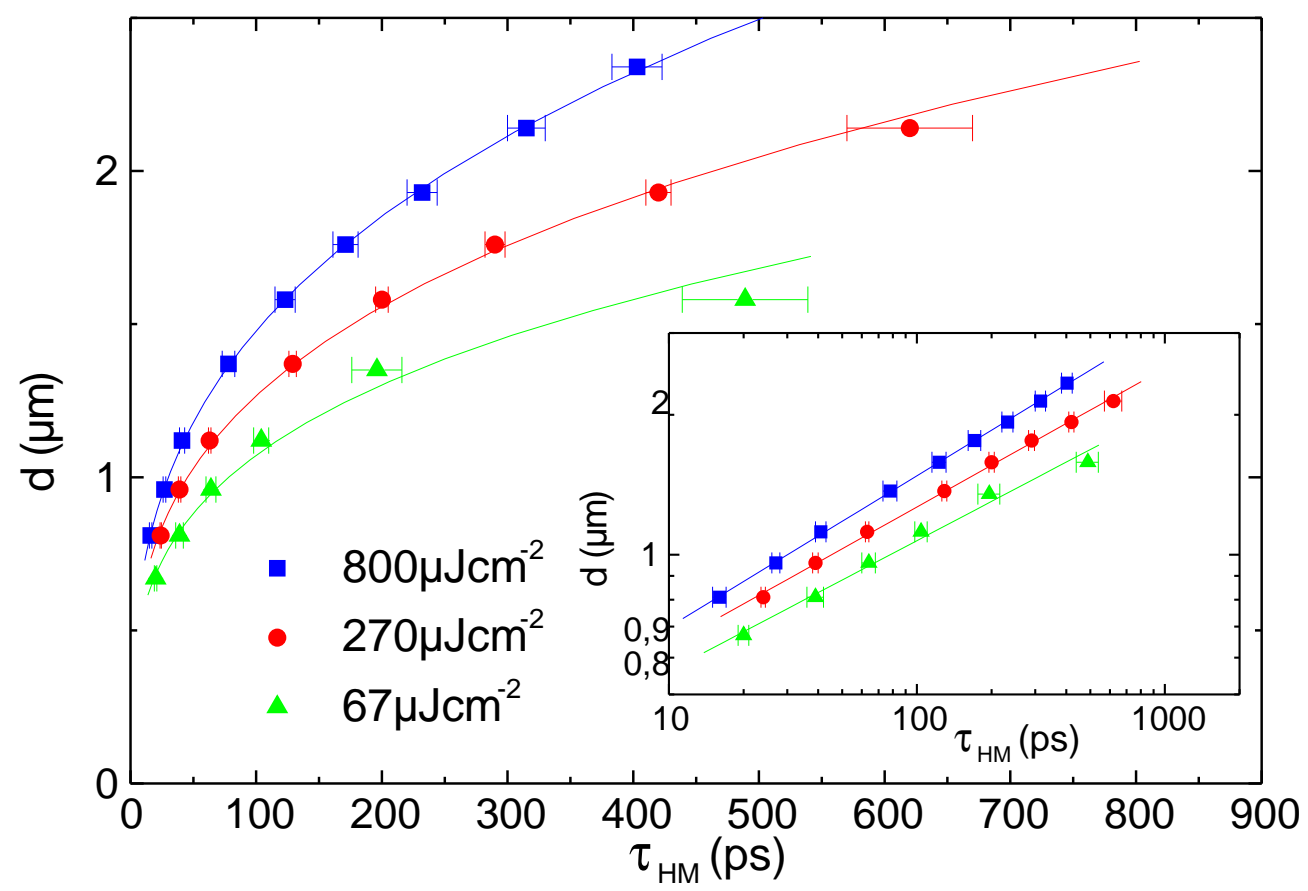

Abbildung 5.8: Probendicke $d$ gegen Ankunftszeit $\tau_{H M}$ für die Fluenzen (Quadrate: $F=800 \mu \mathrm{Jcm}^{-2}$, Kreise: $F=270 \mu \mathrm{Jcm}^{-2}$ und Dreiecke: $F=67 \mu \mathrm{Jcm}^{-2}$ ).

Der Abbildung 5.8 ist zu entnehmen, daß für alle drei Fluenzen ein vergleichbarer Zusammenhang zwischen Ankunftszeit $\tau_{H M}$ und Probendicke $d$ existiert: $d \propto \tau_{H M}^{p}$ mit $p \sim 0,3$.

Somit kann festgestellt werden, daß die Elektron-Loch-Plasma-Ausbreitung in GaAs bei $T_{L}=300 K$ stark von der Fluenz der Pumppulse abhängig ist und daß für unterschiedliche Fluenzen ein vergleichbarer zeitlicher Verlauf der Ausbreitungsgeschwindigkeiten gefunden wird. 


\section{Zusammenfassung der experimentellen Ergebnisse}

Nachdem bei der Messung von $\Delta R_{\text {cis }}(\tau)$ erste Hinweise auf eine schnelle Elektron-LochPlasma-Expansion mit zunehmender Fluenz der Pumppulse gefunden wurden, ist die Plasmaexpansion bei Raumtemperatur mit Hilfe von Flugzeitmessungen detailliert untersucht worden. Bei einer Pumpfluenz von $F=800 \mu \mathrm{Jcm}^{-2}$ wurden relativ scharfe Ladungsträgerfronten gefunden, welche sich mit anfänglich hohen Geschwindigkeiten von $v(\tau=20 \mathrm{ps})=14 \times 10^{5} \mathrm{~cm} / \mathrm{s}$ ausbreiten. Die Ausbreitungsgeschwindigkeit sinkt mit zunehmender Verzögerungszeit $\tau$ auf $v(\tau=400 \mathrm{ps})=2 \times 10^{5} \mathrm{~cm} / \mathrm{s}$. Es wurde folgender Zusammenhang zwischen der Ankunftszeit des EHP-s $\tau_{H M}$ und der Probendicke $d$ gefunden: $d \propto \tau_{H M}^{0,33}$. Aus Laufzeitmessungen mit variabler Pumpphotonenenergie $E_{\text {pump }}$ kann geschlossen werden, daß die untersuchte Plasmaexpansion eher nicht sensitiv auf die anfängliche kinetische Energie der Ladungsträger ist. Die Fluenz der Pumppulse stellte sich als der die Geschwindigkeit der Plasmaexpansion dominierende Parameter heraus. Bei einer festen Probendicke $d$ kann die Korrelation zwischen der Ankunftszeit des Plasmas $\tau_{H M}$ und der Fluenz der Pumppulse $F$ wie folgt beschrieben werden: $\tau_{H M} \propto 1 / F^{0,45}$.

\subsection{Theoretische Beschreibung des Plasma- transportes}

Die experimentellen Befunde aus dem vorherigen Abschnitt 5.1 werfen die Frage nach der Ursache der untersuchten schnellen und dichteabhängigen EHP-Expansion in GaAs auf. Dieser Frage soll in diesem Abschnitt nachgegangen werden.

Es gibt nun unterschiedliche theoretische Konzepte, die räumlich- zeitliche Dynamik von optischen Anregungen in Halbleitern zu beschreiben. Ausgangspunkt ist jeweils eine mikroskopische (Vielteilchen-)Theorie, in welcher die Anregungszustände (Exzitonen, freie Elektronen und Löcher, Phononen, ...) und deren Wechselwirkungen beschrieben werden. Für die Beschreibung von physikalischen Effekten werden neben den vollständig quantenmechanischen Theorien auch semiklassische Theorien, wie die Boltzmann-Transportgleichung angewandt. Eine Übersicht über die quantenmechanische Beschreibung der Anregungszustände in Halbleitern findet man unter anderem in [Hau93]. Eine explizite Lösung auch der semiklassischen Vielteilchentheorien, wie der BoltzmannTransportgleichung für eine Anzahl der beteiligten Teilchen $n>10^{10}$ unter den jeweiligen Anfangs- und Randbedingungen ist explizit i. Allg. nicht möglich. Eine näherungsweise Lösung der Boltzmann-Transportgleichung ermöglichen Molekulardynamik- oder Monte-Carlo-Simulationen [Jac89]. Bei diesen Verfahren ist die Anzahl der simulierten Teilchen als auch die Zeitspanne, über die die Simulation stattfindet, durch die verwendete Rechnerkapazität beschränkt.

Mit einem wesentlich geringeren Rechenaufwand lassen sich sogenannte hydrodynamische Modelle lösen. Bei diesen Modellen wird die zeitliche Entwicklung von den das 
Experiment bestimmenden makroskopischen hydrodynamischen Variablen betrachtet. Die Zeitentwicklung der makroskopischen Variablen wird mit Differentialgleichungen beschrieben, welche man aus Näherungslösungen der mikroskopischen Theorien und entsprechender Mittelung über Einteilchen Variablen über den k-Raum erhält [Kre81, Mah88.

\subsubsection{Hydrodynamisches Diffusionsmodell}

Die in Kap. 5.1 untersuchte Ausbreitung eines Elektron-Loch-Plasmas soll hier im Rahmen eines hydrodynamischen Diffusionsmodells diskutiert werden. Hierbei wird der Transport der optisch angeregten Elektronen und Löcher auf der Basis der BoltzmannTransportgleichung in Relaxationszeitnäherung beschrieben.

\section{Definition des Systems}

Betrachtet werden soll eine planparallele Platte der Dicke $d$ aus GaAs. Es wird angenommen, daß sich die elektrischen und optischen Eigenschaften nur in der Richtung senkrecht zur Probenoberfläche (z-Richtung) ändern (eindimensionales Model[1] ). Die elektrischen Eigenschaften sind durch die in Kap. 2.1 vorgestellte Bandstruktur von GaAs gegeben. Der Einfachheit halber wird nur das Leitungsband und ein Valenzband im Rahmen der Effektivmassenäherung mit $m_{e}^{*}=0,067 m_{e} ; m_{h}^{*}=0,450 m_{e}\left(m_{e}\right.$ : Ruhemasse des freien Elektrons) betrachtet. Durch den Pumppuls werden Elektronen und Löcher mit der lokalen Teilchendichte $n_{e}=n_{h} \equiv n$ instantan zum Zeitpunkt ${ }^{2} t=0$ mit bekanntem Anregungsprofil $n(z) \propto \exp \left[-\alpha_{\text {pump }} z\right]$ angeregt. Eine Renormierung der Einteilchenenergien der angeregten Ladungsträger aufgrund von Austausch und Korrelationseffekten [Zim87] wird hier vernachlässigt (Diskussion siehe Kap. 7). Weiterhin soll angenommen werden, daß die Ladungsträger sich im lokalen thermodynamischen Gleichgewicht befinden, und daß sich die entsprechenden Ladungsträger-Verteilungsfunktionen nicht signifikant auf einer Längenskala ändern, die der mittleren freien Weglänge der Teilchen entspricht. Aufgrund der Tatsache, daß die Ladungsträgerrekombination langsam gegenüber allen anderen hier betrachteten Prozessen ist, werden die Elektronen und Löcher mit Fermi-Verteilungen mit den Quasitemperaturen $T_{e}, T_{h}$ und den Quasiferminiveaus $\psi_{e}$ und $\psi_{h}$ beschrieben, wobei die Quasiferminiveaus bezüglich der jeweiligen Bandkante gemessen werden (siehe Abb. 5.9 .

Desweiteren wird angenommen, daß die Ladungsträger eine konstante Quasitemperatur $T_{e}=T_{h}=300 \mathrm{~K}$ besitzen (isothermischer Transport). Somit werden alle Effekte aufgrund heißer Ladungsträger [Jos90] und heißer Phononen [Dri87] vernachlässigt. Diese Näherung kann zum einen damit motiviert werden, daß die Abkühlung der ursprünglich heißen Ladungsträger mit $T_{c} \gg T_{L}=300 \mathrm{~K}$ auf Raumtemperatur innerhalb einer Zeit

\footnotetext{
${ }^{1}$ Eine eindimensionale Beschreibung ist sinnvoll, da der Fokusdurchmesser des Pumpstrahles von $20 \mu \mathrm{m}$ wesentlich größer als die maximal verwendete Probendicke von $d_{\max }=2,3 \mu \mathrm{m}$ ist.

${ }^{2}$ Diese Näherung ist gerechtfertigt, da die Pulsdauer von $\sim 100 \mathrm{fs}$ wesentlich kürzer ist als die Zeitskala, auf der der hier untersuchte Transport stattfindet.
} 


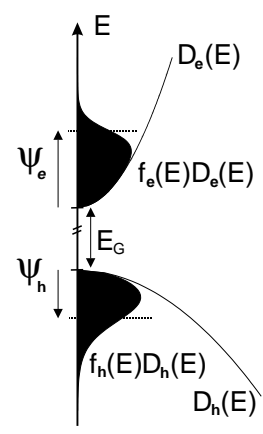

Abbildung 5.9: Definition der Quasiferminiveaus $\psi$ : Schematische Darstellung der Zustandsdichte $D(E)$ und der Besetzungsfunktion $D(E) f(E)$

$<$ 10ps abgeschlossen ist, die klein gegenüber der des untersuchten Ladungstransportes ist (siehe Kap. 2.2). Zum anderen wurde durch die Messungen aus Kap. 5.1.3 gezeigt, daß der untersuchte Transport nicht sensitiv auf die ursprüngliche kinetische Energie der Ladungsträger ist.

\section{Ambipolare Diffusion}

In optisch angeregten Ladungsträgerplasmen wird die gleiche Anzahl von Elektronen und Löchern angeregt. I. Allg. sind die Diffusivitäten von Elektronen und Löcher unterschiedlich, $D_{e} \neq D_{h}$. Unter Vernachlässigung der Wechselwirkung zwischen Elektronen und Löchern würden Elektronen und Löcher unterschiedlich schnell in einem Dichtegradienten diffundieren. Damit würde eine makroskopische Ladungstrennung verbunden sein. Dem entgegen wirkt nun die langreichweitige Coulombwechselwirkung zwischen Elektronen und Löchern, welche eine solche makroskopische Ladungstrennung verhindert 3 , so daß Elektronen und Löcher gemeinsam im Plasma diffundieren. Ein solcher gemeinsamer Transport von Elektronen und Löchern mit $n=n_{e}=n_{h}$ wird als ambipolarer Transport bezeichnet Mah88.

Die Zeitentwicklung der lokalen Ladungsträgerdichte $n$ wird im Rahmen der oben genannten Näherungen durch eine ambipolare Diffusionsgleichung beschrieben [Dri87]:

$$
\frac{\partial n}{\partial t}=\vec{\nabla}(D(n) \cdot \vec{\nabla} n)+G+R
$$

Hierbei ist $R$ die Rekombinationsrate und $G$ die Generationsrate. Die wesentliche Nichtlinearität dieser Gleichung ist in der dichteabhängigen Diffisivität $D=D(n)$ beinhaltet. Man sollte beachten, daß aufgrund der Dichteabhängigkeit der Diffusivität $D$ gilt:

$$
\vec{\nabla}(D(n) \cdot \vec{\nabla} n) \neq D(n) \Delta n .
$$

\footnotetext{
${ }^{3}$ siehe Dembereffekt z.B. in [See92]
} 
Die sich nun anschließende Frage nach der Ursache für die Dichteabhängigkeit der Diffusivität $D(n)$ soll nun im Folgenden beantwortet werden.

\section{Fermidruck: Anschauliche Bedeutung}

Als wesentlichen Effekt, der die Dichteabhängigkeit der Diffusivität verursacht, wird der sogenannte Fermidruck betrachtet. Die Ursache des Fermidruckes liegt darin begründet, daß in einem Fermionischen System mit dem Einsetzen der Entartung das Pauli'sche Ausschließungsprinzip entscheidend wird. Die freie Energie $F_{\text {free }}$ eines entarteten Fermionengases ist signifikant höher, als es im klassischen Gas mit einer Boltzmannverteilung unter sonst gleichen Bedingungen der Fall ist (siehe Abb. 5.10). Die Erhöhung der freien Energie

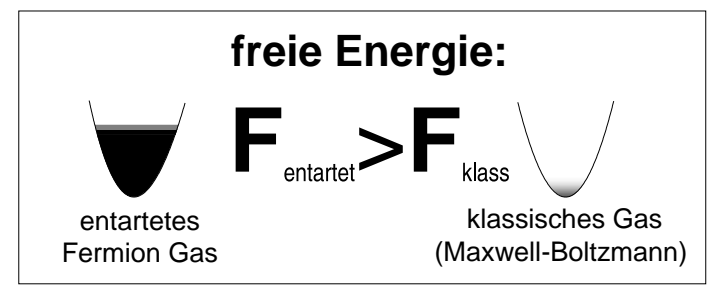

Abbildung 5.10: Schematische Darstellung der Zustandsbesetzung in einem entarteten Fermiongas und einem klassischen Gas bei gleicher Temperatur $T_{c}$ und Dichte $n$

$F_{\text {free }}$ hängt von der Dichte $n$ und somit bei konstanter Teilchenzahl $N$ von dem Volumen $V$ ab. Diese Abhängigkeit kann als Druck interpretiert werden:

$$
p=\left.\frac{\partial F_{\text {free }}}{\partial V}\right|_{N}
$$

Diese obige Ableitung soll nur den in der Literatur [Dri87, Neh96, Kor83, Rom81] so verwendeten Begriff des Fermidruckes veranschaulichen. In der Beschreibung der untersuchten Plasmaexpansion wird dagegen nicht die thermodynamische Variable Druck verwendet, da es sich dort um ein „offenes“ System handelt, in der Volumen und Druck keine Zustandsgrößen sind.

Im folgenden Abschnitt soll nun die Dichteabhängigkeit der Diffusivität $D(n)$ aus der Boltzmann-Transportgleichung unter Verwendung der Fermi-Dirac Statistik abgeleitet werden.

\section{Bestimmung des dichteabhängigen Diffusionskoeffizienten}

Ausgangspunkt der quantitativen Bestimmung von $D=D(n)$ ist die BoltzmannTransportgleichung, welche die zeitliche Entwicklung der Verteilungsfunktion $f_{c}(\mathbf{r}, \mathbf{k}, t)$ 
$(\mathrm{c}=\mathrm{e}, \mathrm{h})$ beschreibt $\mathrm{4}^{4}$.

$$
\frac{d f_{c}}{d t}=\frac{\partial f_{c}}{\partial t}+\dot{\mathbf{k}} \nabla_{\mathbf{k}} f_{c}+\dot{\mathbf{r}} \nabla_{\mathbf{r}} f_{c}=\left.\frac{\partial f_{c}}{\partial t}\right|_{\text {streu }}
$$

Ohne äußere Felder stellt sich für Fermionen die Fermiverteilung $f_{c}^{0}(\mathbf{r}, \mathbf{k})$ als lokale Gleichgewichtsverteilung ein.

$$
f_{c}^{0}(\mathbf{r}, \mathbf{k})=\frac{1}{\exp \left[\left(E(\mathbf{k})-\psi_{c}\right) \beta_{c}\right]+1}
$$

mit $\beta_{c}=1 /\left(k_{b} T_{c}\right)$, der Temperatur der Ladungsträger $T_{c}$ und den Quasiferminiveau $\psi_{c}$.

Im folgenden soll der Ladungsträgertransportes mit Hilfe der Boltzmanngleichung in Relaxationszeitnäherung beschrieben werden. In dieser Näherung wird die Wirkung der Streumechanismen dadurch berücksichtigt, daß sie eine Nichtgleichgewichtsverteilung $f$ auf einer Zeitskala, die mit der Relaxationszeit $\tau_{\text {relax }}$ charakterisiert wird, in eine Gleichgewichtsverteilung $f_{0}$ überführt:

$$
\left.\frac{\partial f_{c}}{\partial t}\right|_{\text {streu }}=-\frac{f_{c}-f_{c}^{0}}{\tau_{\text {relax }}}, \quad f_{c}=f_{c}^{0}+C \exp \left(-t / \tau_{\text {relax }}\right)
$$

Im Rahmen dieser Näherung ist eine explizite Bestimmung der zeitlichen Entwicklung von $f_{c}(\mathbf{r}, \mathbf{k})$ möglich. Die makroskopischen Größen, wie die lokale Ladungsträgerdichte $n_{c}$ und die Stromdichte $j_{c}$, ergeben sich dann wie folgt aus der Verteilungsfunktion $f_{c}(\mathbf{r}, \mathbf{k}, t)$ :

$$
\begin{aligned}
n_{c}(\mathbf{r}, t) & =\int d^{3} \mathbf{k} f_{c}(\mathbf{r}, \mathbf{k}, t) \\
j_{c}(\mathbf{r}, t) & =\frac{\hbar}{m_{c}} \int d^{3} \mathbf{k} \mathbf{k} f_{c}(\mathbf{r}, \mathbf{k}, t)
\end{aligned}
$$

Hierbei ist $m_{c}$ die effektive Masse der Elektronen $(\mathrm{c}=\mathrm{e})$ bzw. Löcher $(\mathrm{c}=\mathrm{h})$.

Für die Beschreibung des Experimentes wird angenommen, daß sich die Ladungsträger lokal im thermodynamischen Gleichgewicht befinden und mit der Fermiverteilungsfunktion (Gleichung 5.5) mit $T_{c}=$ konst. $=300 \mathrm{~K}$ beschrieben werden. Die anfänglich optisch generierte unterschiedliche Ladungsträgerdichte $n_{c}(z)$ hat eine Ortsabhängigkeit der Quasiferminiveaus $\psi_{c}(z)$ zur Folge. Die Abhängigkeit von $n_{c}$ und $\psi_{c}$ erhält man durch Einsetzen von Gl. (5.5) in Gl. (5.7):

$$
n_{c}=2\left[\frac{m_{c} \beta}{2 \pi \hbar^{2}}\right]^{3 / 2} \mathcal{F}_{1 / 2}\left(\beta \psi_{c}\right),
$$

mit dem n-ten Fermiintegral $\mathcal{F}_{n}$ :

$$
\mathcal{F}_{n}(z)=\frac{1}{\Gamma(n+1)} \int_{0}^{\infty} \frac{x^{n}}{\exp (x-z)+1} d x .
$$




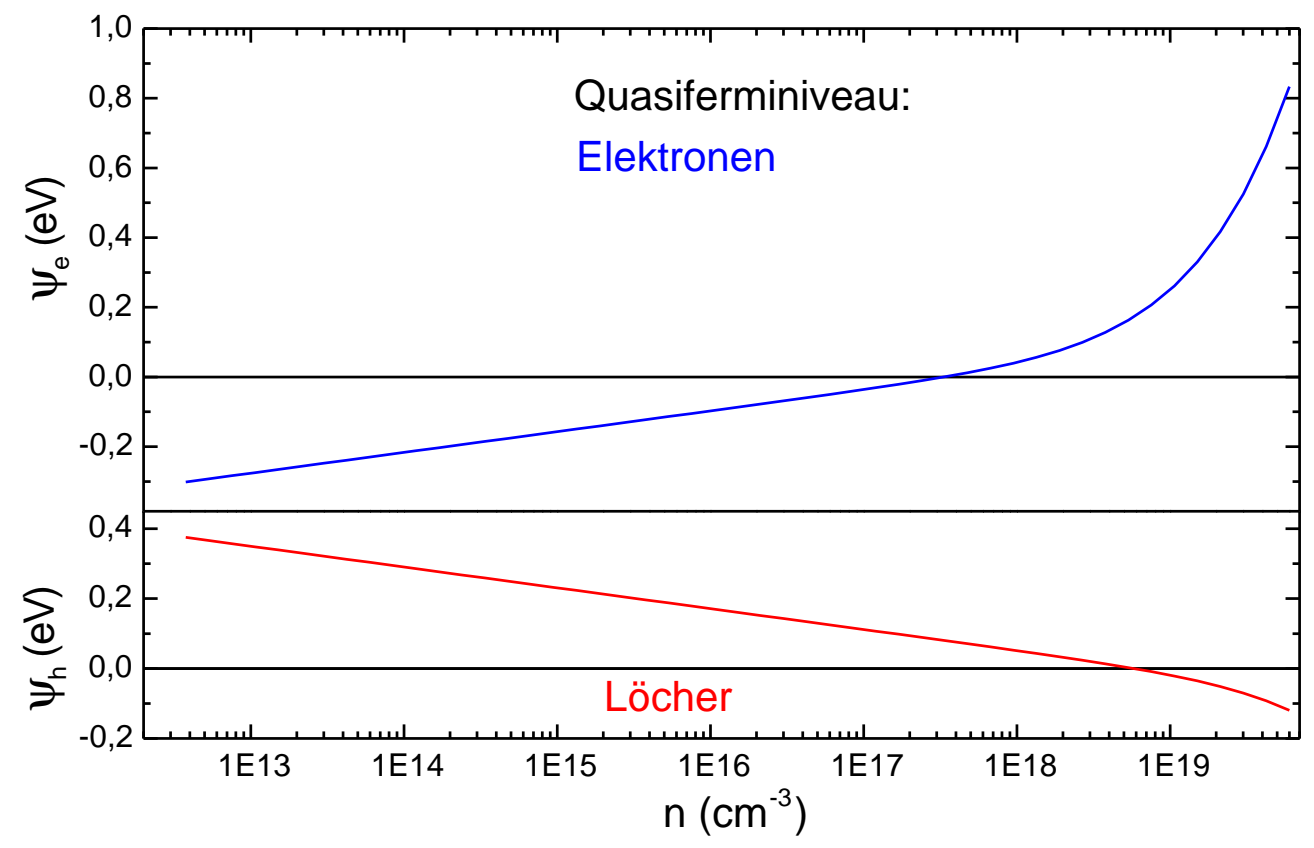

Abbildung 5.11: Quasiferminiveaus der Elektronen und Löcher als Funktion des Logarithmus der Teilchendichte $n$ bei $T_{c}=T_{e}=T_{h}=300 \mathrm{~K}$

Die Fermiintegrale lassen sich außer für $n=0$ nicht analytisch lösen. In dieser Arbeit wurden deshalb die Integrale numerisch berechnet, oder es wurden explizite Näherungslösungen aus [Zim87] verwendet. In der Abbildung 5.11] sind die Quasiferminiveaus $\psi_{c}$ als Funktion der Ladungsträgerdichte $\log n_{c}$ aufgetragen. Für kleine Dichten (nicht entartetes Fermigas) liegen die Quasiferminiveaus im Bereich der Bandlücke und steigen betragsmäßig mit $\left|\psi_{c}\right| \propto \log n_{c}$ an. Beim Übergang vom nicht entarteten zum entarteten Fermigas wechselt $\psi_{c}$ das Vorzeichen. Dieses geschieht bei den Elektronen bei $n_{e} \sim 3 \times 10^{17} \mathrm{~cm}^{-3}$ und bei den Löchern bei $n_{h} \sim 6 \times 10^{18} \mathrm{~cm}^{-3}$. Dieser Unterschied wird durch die größere effektive Masse der Löcher gegenüber der der Elektronen verursacht. Im entarteten Grenzfall steigt $\left|\psi_{c}\right|$ mit $\left|\psi_{c}\right| \propto n_{c}^{2 / 3}$. Der starke Anstieg von $\left|\psi_{c}(n)\right|$ mit dem Einsetzen der Entartung ist Ausdruck des Fermidruckes. Während die $\left|\psi_{c}\right| \propto \log n_{c}$ Abhängigkeit im nicht entarteten Fall zu einem dichteunabhängigen Diffusionskoeffizienten $D(n)=$ konst. führt, ist die $\left|\psi_{c}\right| \propto n_{c}^{2 / 3}$ Abhängigkeit für das entartete Fermigas schließlich für eine mit der Dichte $n$ zunehmende Diffusivität verantwortlich.

Als Lösung der Boltzmann-Transportgleichung erhält man unter den oben beschriebenen Näherungen eine Stromdichte $j_{c}$, welche proportional zu dem Gradienten des Quasiferminiveaus $\psi_{c}$ ist [Dri87, Kre81] (siehe Abb. 5.12).

\footnotetext{
${ }^{4}$ Eine Ableitung der Boltzmanngleichung und Diskussion über ihre Anwendbarkeit findet man in Standard-Textbüchern, z.B. auch in [Mad96]
} 


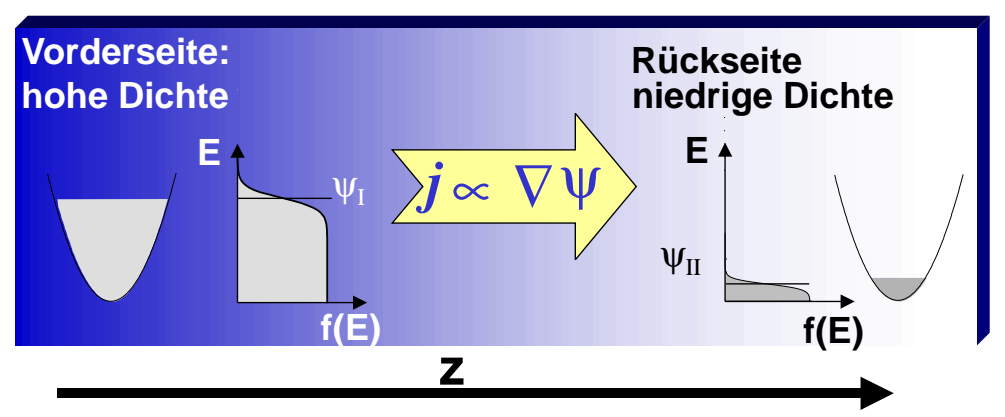

Abbildung 5.12: Schematische Darstellung der Probe mit einem Gradienten der Dichte bzw. des Quasiferminiveaus und der daraus resultierenden Stromdichte $j$

$$
j_{c}=\frac{1}{q} \sigma_{c} \nabla \psi_{c}
$$

Hierbei ist $\sigma_{c}(\mathrm{c}=\mathrm{e}, \mathrm{h})$ die dichteabhängige elektrische Leitfähigkeit der Elektronen bzw. Löcher:

$$
\sigma_{c}=e n_{c} \mu_{c}^{0} \frac{\mathcal{F}_{0}\left(\beta \psi_{c}\right)}{\mathcal{F}_{1 / 2}\left(\beta \psi_{c}\right)}
$$

mit der Beweglichkeit $\mu_{c}^{0}$ der Ladungsträger für eine Maxwell-Boltzmann Verteilung. Für den Fall des ambipolaren Transportes $\left(j_{e}=-j_{h}, n=n_{e}=n_{h}\right)$ der Elektronen und Löcher gemeinsam ergibt sich für den Elektron-Loch-Paar-Teilchenstrom $J$ :

$$
\begin{aligned}
J & =-\frac{1}{q} j_{e}=\frac{1}{q} j_{h} \\
& =\frac{1}{q^{2}} \frac{\sigma_{e} \sigma_{h}}{\sigma_{e}+\sigma_{h}}\left[\nabla\left(\psi_{h}-\psi_{e}\right)\right]
\end{aligned}
$$

Da die Quasiferminiveaus $\psi_{c}$ über Gl. (5.8) mit der lokalen Dichte n zusammenhängen, kann man den Gradienten der Quasiferminiveaus $\nabla\left(\psi_{h}-\psi_{e}\right)$ in einen Dichtegradienten $\nabla n$ umrechnen. Man erhält (siehe Anhang):

$$
J=-\frac{1}{q^{2}} \frac{1}{n \beta} \frac{\sigma_{e} \sigma_{h}}{\sigma_{e}+\sigma_{h}}\left[\frac{\mathcal{F}_{1 / 2}\left(\beta \psi_{h}\right)}{\mathcal{F}_{-1 / 2}\left(\beta \psi_{h}\right)}+\frac{\mathcal{F}_{1 / 2}\left(\beta \psi_{e}\right)}{\mathcal{F}_{-1 / 2}\left(\beta \psi_{e}\right)}\right] \nabla n
$$

Vergleicht man Gl. (5.13) nun mit der Diffusionsgleichung $J=-D(n) \nabla n$ so erhält man den dichteabhängigen ambipolaren Diffussionskoeffizienten:

$$
D=\frac{1}{q^{2}} \frac{1}{n \beta} \frac{\sigma_{e} \sigma_{h}}{\sigma_{e}+\sigma_{h}}\left[\frac{\mathcal{F}_{1 / 2}\left(\beta \psi_{h}\right)}{\mathcal{F}_{-1 / 2}\left(\beta \psi_{h}\right)}+\frac{\mathcal{F}_{1 / 2}\left(\beta \psi_{e}\right)}{\mathcal{F}_{-1 / 2}\left(\beta \psi_{e}\right)}\right]
$$




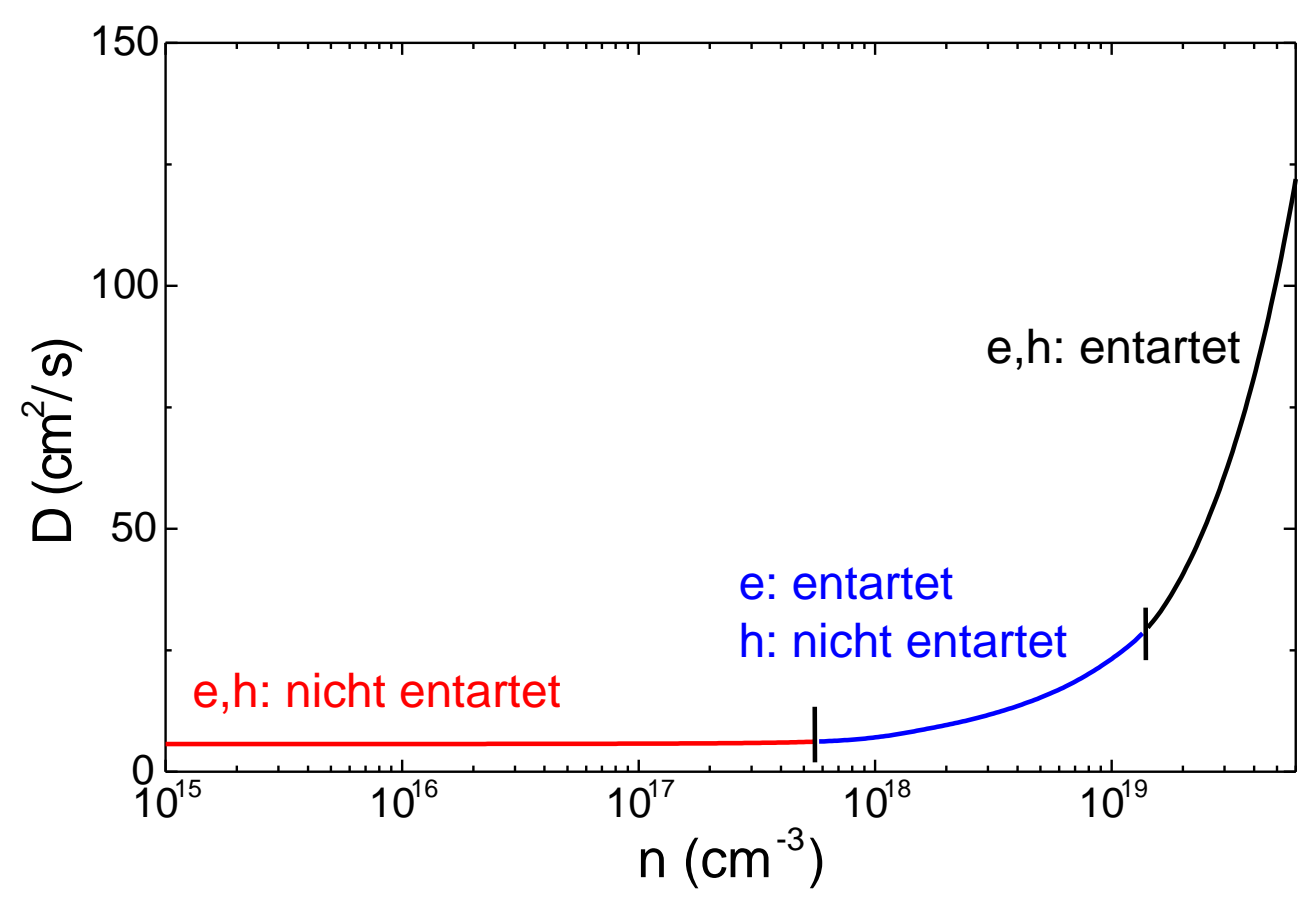

Abbildung 5.13: Dichteabhängige Diffusivität für GaAs bei $T_{c}=T_{L}=300$ mit den Niedrigdichte-Diffusionskoeffizienten $D_{e}^{0}=100 \mathrm{~cm}^{2} / s ; D_{h}^{0}=3 \mathrm{~cm}^{2} / s$

\section{Diskussion des dichteabhängigen Diffusionskoeffizienten}

Für die Materialparameter von GaAs bei einer Temperatur von $T=T_{L}=T_{c}=300 \mathrm{~K}$ ist die Dichteabhängigkeit der Diffusivität $D=D(n)$ in der Abb. 5.13 dargestellt. Man erkennt, daß für kleine Dichten (nicht entartetes Fermigas) der Diffusionskoeffizient konstant ist. Dieser ambipolare Niedrigdichte-Diffusionskoeffizient $D_{a m b}^{0}$ erfüllt die Einsteinbeziehung für ein nicht entartetes Ladungsträgergas:

$$
D_{a m b}^{0}=\frac{2 D_{e}^{0} D_{h}^{0}}{D_{e}^{0}+D_{h}^{0}} ; \quad D_{c}^{0}=\frac{\mu_{c}^{0}}{q \beta}
$$

Bei einer Dichte von $n \approx 3 \times 10^{17} \mathrm{~cm}^{-3}$ setzt die Entartung der Elektronen (Vgl. Abb. 5.11 und bei $n \approx 3 \times 10^{17} \mathrm{~cm}^{-3}$ die der Löcher ein. Mit dem Einsetzen der Entartung ist eine Erhöhung des Diffusionskoeffizienten $D(n)$ verbunden. Diese Dichteabhängigkeit des Diffusionskoeffizienten $D(n)$ wird durch die Fermiintegrale $\mathcal{F}_{j}(\beta \psi) / \mathcal{F}_{k}(\beta \psi)$ aus Gl. (5.14) und durch die Dichteabhängigkeit der elektrischen spezifischen Leitfähigkeit $\sigma_{c}(n)$ (siehe Gl. (5.11) verursacht. Im entarteten Grenzfall $(\beta \psi \gg 1)$ können die Fermiintegrale explizit gelöst werden und man erhält [Pol96]:

$$
\psi_{c}=\frac{\hbar^{2}\left(3 \pi^{2} n_{c}\right)^{2 / 3}}{2 m_{c}},
$$




$$
\frac{\mathcal{F}_{0}\left(\beta \psi_{c}\right)}{\mathcal{F}_{1 / 2}\left(\beta \psi_{c}\right)}=\frac{3 \sqrt{\pi}}{4}\left(\beta \psi_{c}\right)^{-1 / 2}, \frac{\mathcal{F}_{1 / 2}\left(\beta \psi_{c}\right)}{\mathcal{F}_{-1 / 2}\left(\beta \psi_{c}\right)}=\frac{2}{3} \beta \psi_{c}
$$

Im entarteten Fall steigt $\sigma_{c}$ nur noch mit $\sigma_{c} \propto n^{2 / 3}$ statt mit $\sigma_{c} \propto n$ im nicht entarteten Fall an. Dieses Verhalten ist darin begründet, daß im entarteten Fall nur noch Ladungsträger an der Fermifläche zum Transport beitragen. Die treibende Kraft des Fermidruckes führt zu den $\mathcal{F}_{j}(\beta \psi) / \mathcal{F}_{k}(\beta \psi)$-Termen in Gl. (5.14), die bei genügend hohen Dichten proportional zu $n^{2 / 3}$ sind.

\subsubsection{Numerische Simulation}

Mit dem im vorherigen Abschnitt bestimmten dichteabhängigen Diffusionskoeffizienten $D=D(n)$ wird nun die ambipolare Diffusionsgleichung (5.1) numerisch in einer Dimension gelöst (Zeitschritte: $\Delta t<10 \mathrm{fs}$; Anzahl der örtlichen Stützstellen: $>100$ ).

$$
\frac{\partial n}{\partial t}=\vec{\nabla}(D(n) \cdot \vec{\nabla} n)+G+R
$$

Hierbei wird die Diffusivität für den örtlichen Stützpunkt $i$ bei dem Zeitschritt $j$ als $D\left(n_{i}^{j-1}\right)$ gesetzt. Zum Zeitpunkt $\tau=0$ wird eine exponentiell mit der Absorptionslänge des Pumppulses abfallende Ladungsdichteverteilung angenommen:

$$
n(z, \tau=0)=n_{0} \cdot \exp \left[-\alpha_{\text {pump }} z\right]
$$

Für die Ladungsträgerrekombination $R$ wird Gl. 2.9 aus Kap. 2.3 verwendet, wobei nur die Auger- und bimolekulare Rekombination mit den dort angegebenen Koeffizienten berücksichtigt werden:

$$
R=-B n^{2}-C n^{3}
$$

Für die Randbedingung wurden der Einfachheithalber vollständig reflektierende Oberflächen angenommen 5 . Für den Vergleich der simulierten räumlich und zeitlichen Dynamik der Ladungsträgerdichte $n(\tau, z)$ mit den experimentellen Daten wurde angenommen, daß das $\Delta R_{\text {trans }}(\tau)$ Signal proportional zur Ladungsträgerdichte $n=n(z=d)$ ist ${ }^{6}$.

In der Abbildung 5.14 ist die simulierte räumlich-zeitliche Dynamik der Ladungsträgerdichte $n(z, \tau)$ dargestellt. Man erkennt, daß die ursprünglich hohe Dichte bei $z=0$ sehr schnell auf einer Zeitskala $\sim 1$ ps abnimmt. Diese schnelle Abnahme wird nicht nur

\footnotetext{
${ }^{5}$ Der Einfluß der Randbedingungen, insbesondere der der Oberflächenrekombinationsgeschwindigkeit, wurde ausführlich in Hil91] und Mah88 untersucht. Um den Einfluß der Randbedingungen auf die Ergebnisse der hier durchgeführten Simulation zu überprüfen, wurde eine Oberflächenrekombinationsgeschwindigkeit als variabler Parameter in den Simulationen eingesetzt. Es hat sich gezeigt, daß die Dichteabhängigkeit des Plasmatransportes nicht signifikant von der Größe dieser Oberflächenrekombinationsgeschwindigkeit abhängig ist.

${ }^{6}$ In Wit99] wurde gezeigt, daß diese Annahme unter den hier vorliegenden Bedingungen sinnvoll ist. Jedoch kann unter dieser Annahme der für kleine Probendicken beobachtete instantane Anstieg der $\Delta R_{\text {trans }}$ Kurven (siehe z.B. Abb. 5.2 nicht reproduziert werden (siehe auch Diskussion in Kap. 6.3.2).
} 


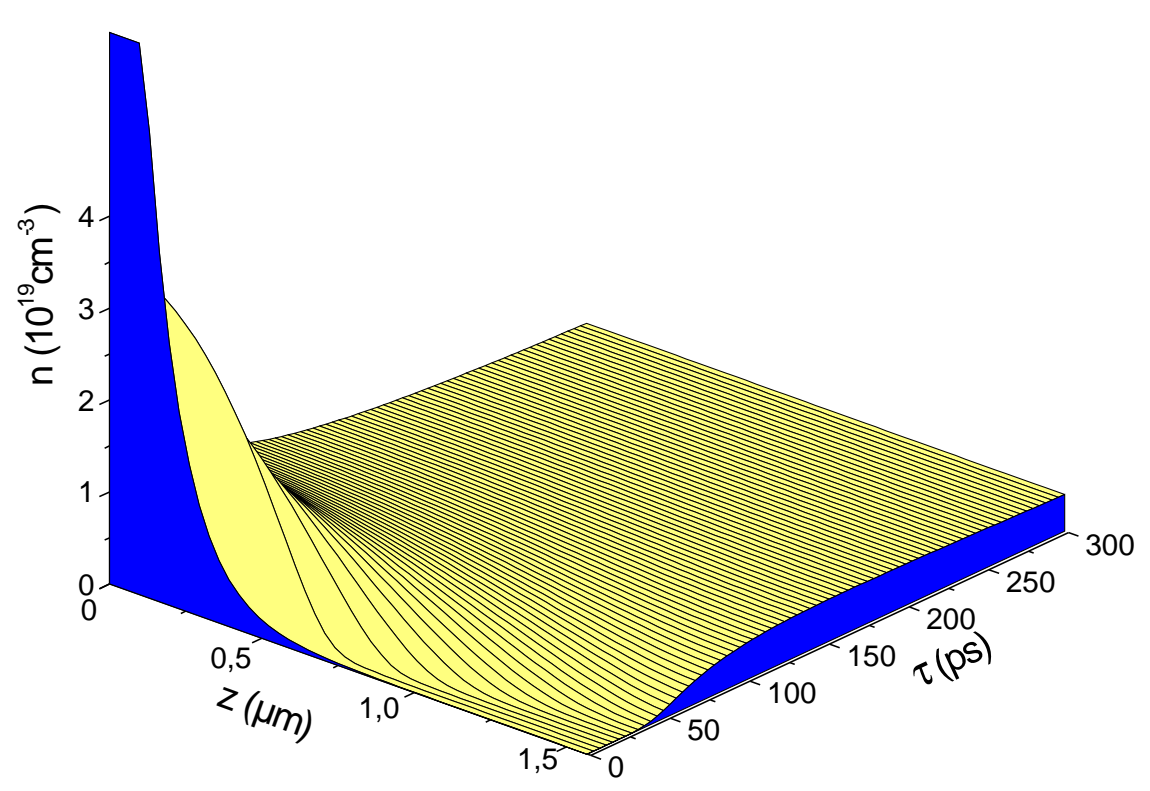

Abbildung 5.14: 3-dimensionale Darstellung der simulierten zeit- und ortsabhängigen Ladungsträgerverteilung. Die anfängliche Ladungsträgerdichte entspricht einer Fluenz von $F=800 \mu \mathrm{Jcm}^{-2}$. Probendicke $d=1,6 \mu \mathrm{m}$

von einem schnellen Transport der Ladungsträger in die Tiefe der Probe, sondern auch von der dichteabhängigen Auger und Plasmarekombination (siehe Gl. (2.9)) verursacht.

Die anfänglich örtlich inhomogene Ladungsträgerverteilung relaxiert hier innerhalb von ca. 100ps mittels der dichteabhängigen Diffusion in eine örtlich homogene Ladungsträgerverteilung. Danach nimmt nur noch die Ladungsträgerdichte insgesamt durch Ladungsträgerrekombination ab.

\subsection{Vergleich Experiment - Simulation}

In diesem Abschnitt findet der Vergleich von den Ergebnissen der Simulationsrechnungen mit den experimentellen Ergebnissen zur Elektron-Loch-Plasma-Ausbreitung in GaAs bei $T_{L}=300 \mathrm{~K}$ statt. Speziell wird folgendes verglichen:

- der Verlauf des $\Delta R_{\text {trans }}(\tau)$ Signals für eine Probendicke von 1, $9 \mu \mathrm{m}\left(F=800 \mu \mathrm{Jcm}^{-2}\right)$ mit der Simulation mit dem von der lokalen Dichte n(z) abhängigen Diffusionskoeffizienten $D=D(n(z))$ und mit einer Simulation mit einem konstanten Diffusionskoeffizienten $\mathrm{D}=$ konst.,

- $\Delta R_{\text {trans }}(\tau)$ bei Variation der Probendicke $d$ bei $F=800 \mu \mathrm{Jcm}^{-2}$ mit Simulation mit $\mathrm{D}=\mathrm{D}(\mathrm{n}(\mathrm{z}))$

- $\Delta R_{\text {trans }}(\tau)$ bei Variation der Fluenz $F$ bei $d=1,0 \mu \mathrm{m}$ mit Simulation mit $\mathrm{D}=\mathrm{D}(\mathrm{n}(\mathrm{z}))$ 
Alle Simulationen mit $D=D(n(z))$ wurden mit dem identischen dichteabhängigen Diffusionskoeffizienten $D(n)$ (siehe Abb. 5.13) durchgeführt. Ein Vergleich der Simulationsrechnungen mit den $\Delta R_{c i s}$ Kurven wird nicht vorgenommen, da die $\Delta R_{c i s}$ Signale von der energetischen Relaxation der Ladungsträger dominiert werden, welche in der Simulation vernachlässigt wurde.

\subsubsection{Vergleich des Verlaufs von $\Delta R_{\text {trans }}(\tau)$ mit Simulationsrech- nungen mit $\mathrm{D}=\mathrm{D}(\mathrm{n}(\mathrm{z}))$ und $\mathrm{D}=$ konst.}

Das einfachst mögliche Modell, um einen dichteabhängigen Ladungstransport zu beschreiben, besteht darin, den Diffusionskoeffizienten als konstanten Parameter in der Diffusionsgleichung (Gl. (5.1)) einzusetzen. Die Größe eines solchen Diffusionskoeffizienten kann man als Fitparameter für die unterschiedlichen Fluenzen $F$ bzw. Anregungsdichten $n_{0}$ benutzen. In der Abbildung 5.15 sind die berechneten Dichteprofile $\mathrm{n}(\mathrm{z})$ für $\tau=0 ; 5 ; \ldots ; 20 \mathrm{ps}$ aufgetragen, welche zum einen mit einem konstanten Diffusionskoeffizienten und zum anderen mit einem von der lokalen Dichte abhängigen Diffusionskoeffizienten $D=D(n(z))$ bestimmt wurden. Der konst. Diffusionskoeffizient wurde hier so gewählt, daß die Ankunftszeit $\tau_{H M}$ der Ladungsträger bei einer Probendicke von $d=1,9 \mu \mathrm{m}$ für beide Simulationen identisch ist (siehe Abb. 5.16). Bei den mit $D=D(n(z)$ ) berechneten Kurven kann man im Vergleich zu den mit einem konstanten Diffusionskoeffizienten bestimmten Kurven eine deutlich schnellere Abnahme der Dichte bei $z \approx 0$ feststellen.

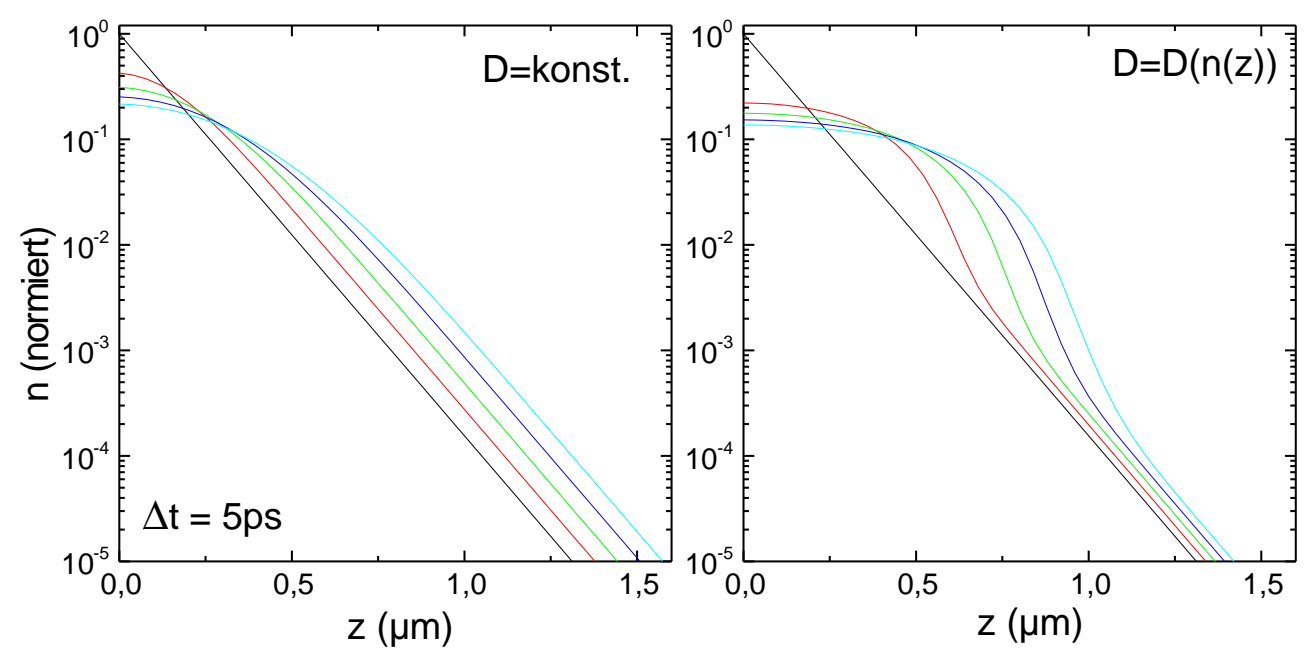

Abbildung 5.15: Dichteprofile $n(z)$ für Verzögerungszeiten $\tau=0 ; 5 ; \ldots ; 20$ ps in logarithmischer Auftragung simuliert mit $D=$ konst. und $D=D(n(z))$

Bei $D=D(n(z))$ ist eine Ausbildung einer relativ scharfen Ladungsträgerfront zu erkennen, die dadurch zustande kommt, daß die schnell diffundierenden Ladungsträger innerhalb des Hochdichte-Plasmas von langsam diffundierenden Ladungsträgern an der Vorderfront des Plasmas zurückgehalten werden. 
Die Ausbildung einer relativ scharfen Ladungsträgerfront ist nicht nur in den zeitabhängigen Dichteprofilen (die im Experiment nicht zugänglich sind) zu erkennen, sondern spiegelt sich auch im zeitlichen Verlauf der Dichte bei $z=d$ wieder, die man mit der Messung von $\Delta R_{\text {trans }}(\tau)$ vergleichen kann. Ein solcher Vergleich wird in der Abbildung 5.16 vorgenommen. Aufgetragen sind dort die $n(\tau, z=d)$ Kurven bei $d=1,9 \mu \mathrm{m}$, berechnet mit $D=$ konst. und $D=D(n(z)$ ) (gleiche Parameter wie Abb. 5.15) und das entsprechende $\Delta R_{\text {trans }}(\tau)$ Meßsignal aus Abschnitt 5.1.2.

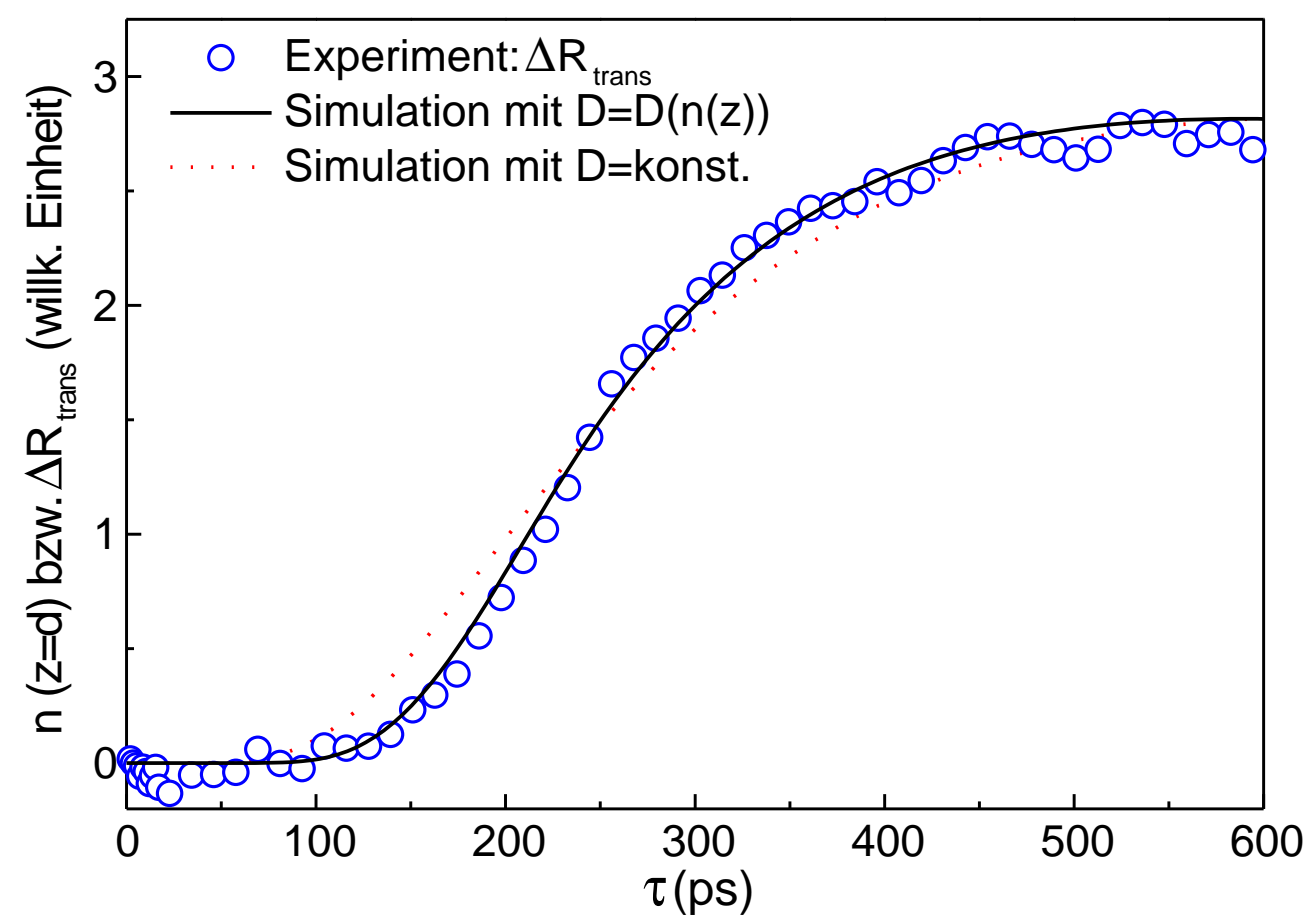

Abbildung 5.16: Vergleich von Experiment: $\Delta R_{\text {trans }}(\tau)$ bei $d=1,9 \mu m F=800 \mu \mathrm{Jcm}^{-2}$ $E_{\text {pump }}=2,44 \mathrm{eV}$ mit Simulation mit $D=$ konst. und $D=D(n(z))$

Der Vergleich der Simulationen mit $D=$ konst. und $D=D(n(z)))$ zeigt, daß die Ausbreitung einer relativ scharfen Ladungsträgerfront einen signifikant steileren Anstieg der $n(\tau, z=d)$ Kurven zur Folge hat. Die Simulation mit $D=k o n s t$. liefert auch unter Variation aller in der Simulation verwendeten Parameter nicht einen solch steilen Anstieg. Der Vergleich der beiden Simulationen mit der experimentellen Kurve zeigt, daß die Simulation mit $D=D(n(z))$ eine deutlich bessere Übereinstimmung mit dem Experiment aufweist.

Die gute Übereinstimmung des Kurvenverlaufs von $n(\tau, z=d$ ) (simuliert bei $D=D(n(z)))$ mit dem experimentell gemessenen $\Delta R_{\text {trans }}(\tau)$ Signal bei einer Probendicke $d=1,9 \mu \mathrm{m}$ läßt auf eine Ausbildung einer relativ scharfen Ladungsträgerfront bei der Elektron-Loch-Plasma-Expansion in GaAs schließen. 


\subsubsection{Vergleich Experiment - Simulation: Variation der Proben- dicke}

In der Abbildung 5.17 sind die experimentellen $\Delta R_{\text {trans }}(\tau)$ Kurven aus Abschnitt 5.1 .2 zusammen mit den simulierten Kurven $(D=D(n(z)))$ für unterschiedliche Probendicken $d$ aufgetragen. Man erkennt, daß die berechneten Kurven relativ gut sowohl die Zeitabhängigkeit der einzelnen $\Delta R_{\text {trans }}(\tau)$ Kurven als auch die Abhängigkeit der $\Delta R_{\text {trans }}(\tau)$ Signale von der Probendicke $d$ beschreiben. Auch die schnelleren Abfallzeiten der $\Delta R_{\text {trans }}(\tau)$ Signale mit abnehmender Probendicke werden von der Simulation gut wiedergegeben. Dieses Verhalten wird von der dichteabhängigen Ladungsträgerrekombination verursacht.

Eine gute Übereinstimmung von Experiment mit der Theorie mit $D=D(n(z))$ ist auch für die Abhängigkeit der Ankunftszeit der Ladungsträger $\tau_{H M}$ von der Probendicke $d$ festzustellen (siehe Inset von Abb. 5.17). Aus der Theorie mit $D=D(n(z)$ ) erhält man folgenden Zusammenhang von $\tau_{H M}$ und Probendicke $d$ :

$$
\text { Theorie mit } D=D(n(z)): d \propto t_{H M}^{0,36},
$$

welches vergleichbar mit der experimentell gefundenen Korrelation von

$$
\text { Experiment }: d \propto \tau_{H M}^{0,33}
$$

ist. Dahingegen liefert die Simulation mit einem konstanten Diffusionskoeffizienten $D=$ konst. einen Zusammenhang von $d \propto \tau_{H M}^{0,5}$, welcher stark von der gemessenen Korrelation abweicht.

Somit kann festgestellt werden, daß der experimentell gefundene zeitliche Verlauf der Ausbreitungsgeschwindigkeit von der Simulation mit $D=$ konst. nur unbefriedigend beschrieben wird. Eine wesentlich bessere Übereinstimmung mit dem Experiment wird von der Simulation mit $D=D(n(z))$ erzielt. 


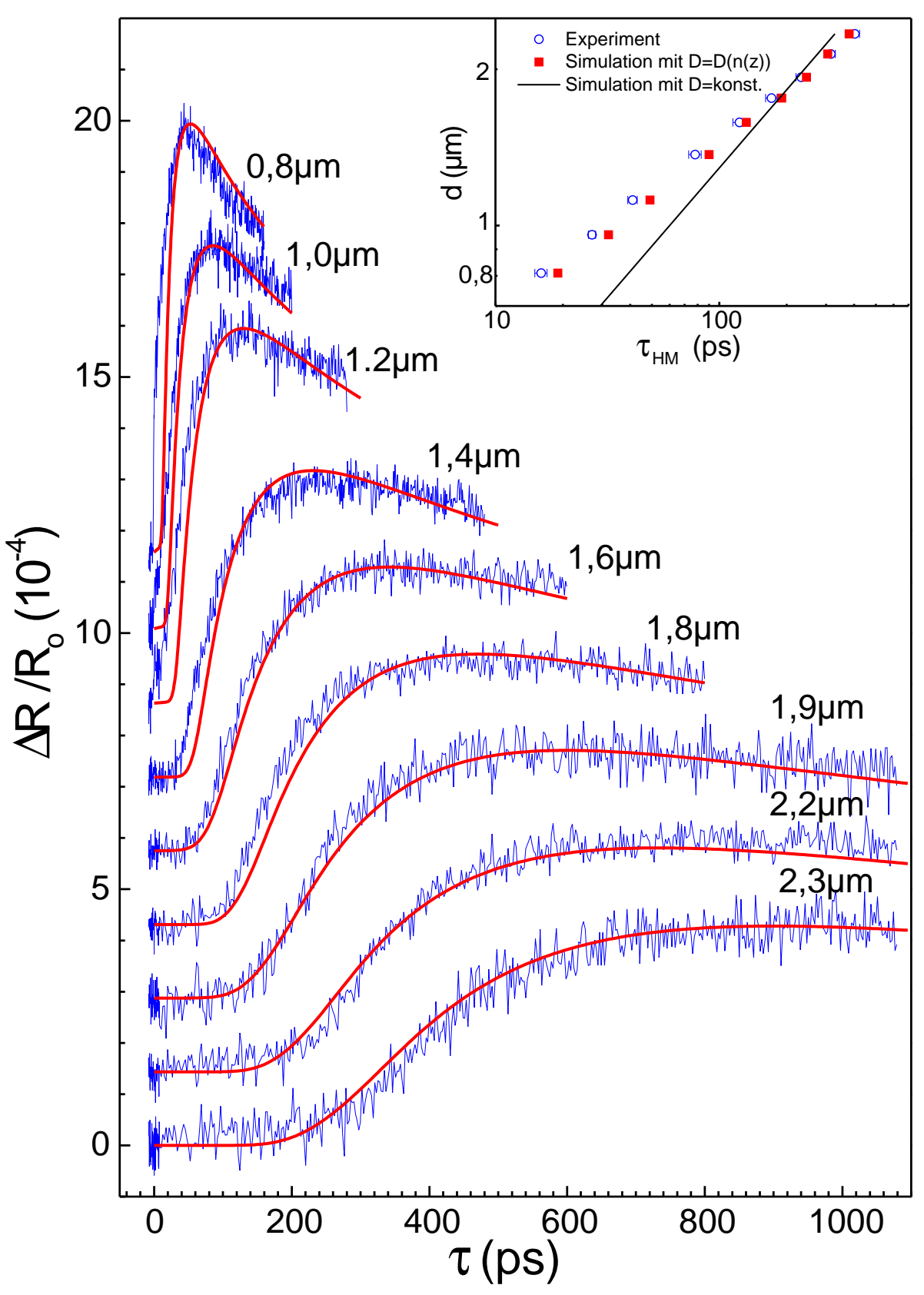

Abbildung 5.17: Variation der Probendicke: Vergleich von Theorie mit $\mathrm{D}=\mathrm{D}(\mathrm{n}(\mathrm{z}))$ mit dem Experiment bei $F=800 \mu \mathrm{Jcm}^{-2}$ welches mit einer anfänglichen Ladungsträgerdichte von $n_{0}=1,2 \times 10^{2} 0 \mathrm{~cm}^{-3}$ entspricht. Im Inset ist die Abhängigkeit der Ankunftszeit $\tau_{H M}$ von der Probendicke $d$ auf einer doppellogarithmischen Skala für das Experiment und Simulation mit $D=D(n(z))$ und mit $D=$ konst. aufgetragen. 


\subsubsection{Vergleich Experiment - Simulation: Variation der Pump- fluenz}

In der Abbildung 5.18 sind die theoretischen und experimentellen Kurven für $\Delta R_{\text {trans }}(\tau)$ für unterschiedliche Pumpfluenzen bei konstanter Dicke $d$ aufgetragen. Auch hier werden die wesentlichen Ergebnisse der experimentellen Untersuchung recht gut von der Modellsimulation beschrieben. Die mit der Simulation bestimmten Ankunftszeiten $\tau_{H M}$ stimmen bis auf die bei der höchsten Fluenz von $F=800 \mu \mathrm{Jcm}^{-2}$ innerhalb des experimentellen Fehlers mit den experimentellen Zeiten überein (siehe inset von Abb. 5.18). Die Simulation liefert folgenden Zusammenhang von Ankunftszeit $\tau_{H M}$ und Fluenz $F$ :

$$
\text { Theorie }: \tau_{H M} \propto F^{-0,56} \text {, }
$$

welches vergleichbar ist mit dem experimentell gefundenen Zusammenhang von:

$$
\text { Experiment }: \tau_{H M} \propto F^{-0,45} \text {. }
$$

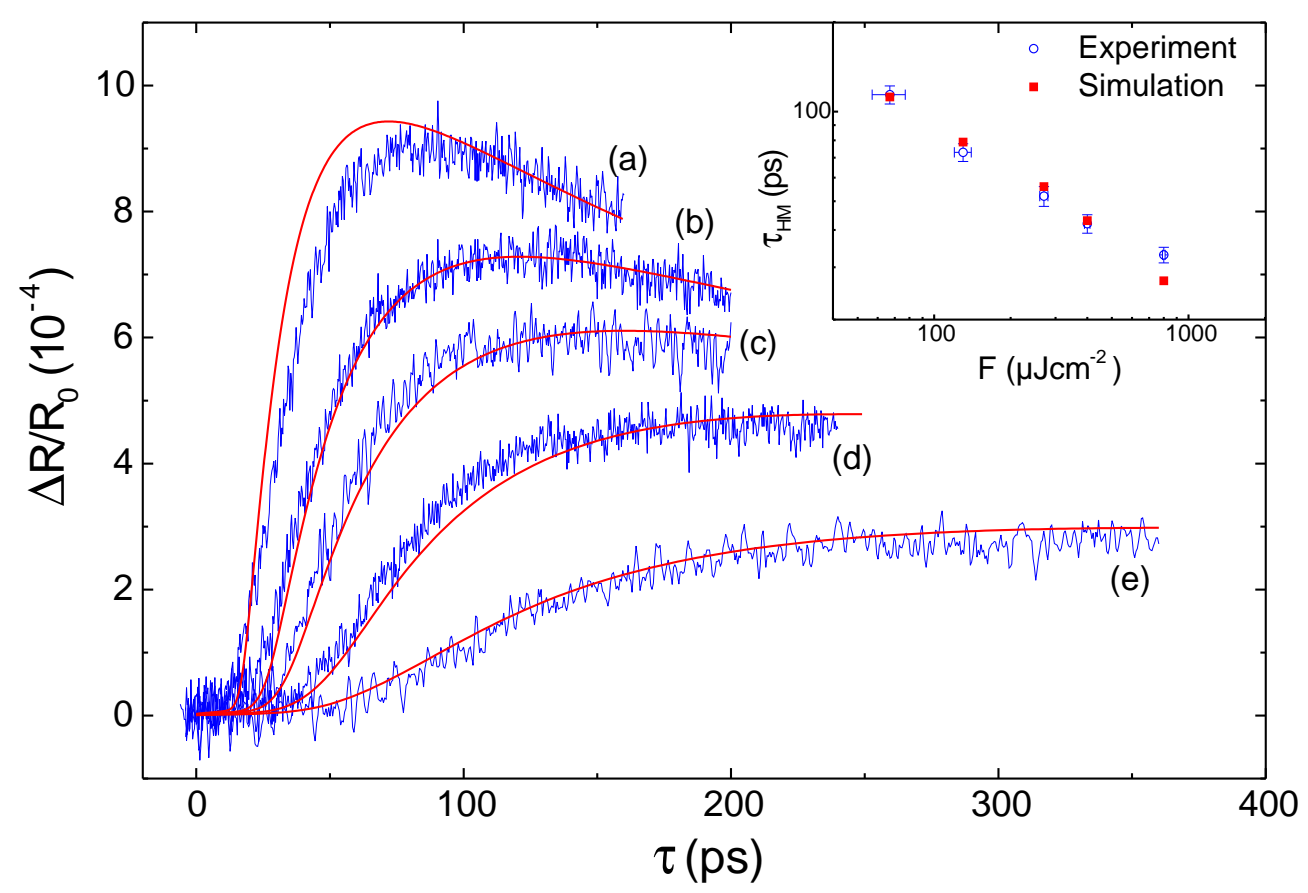

Abbildung 5.18: Vergleich Experiment mit Simulation bei Variation der Pumpfluenz $F$ bei $d=1,0 \mu \mathrm{m}\left(E_{\text {pump }}=E_{G}+1 \mathrm{eV}\right)$. (a) $F=800 \mu \mathrm{Jcm}^{-2}$, (b) $F=400 \mu \mathrm{Jcm}^{-2}$, (c) $F=270 \mu \mathrm{Jcm}^{-2}$, (d) $F=130 \mu \mathrm{Jcm}^{-2}$ und (e) $F=67 \mu \mathrm{Jcm}^{-2}$. Im Inset ist $\tau_{H M}$ für Experiment und Theorie als Funktion von der Fluenz doppellogarithmisch aufgetragen. 
Die gute Übereinstimmung der Modellsimulation mit dem Experiment läßt die Schlußfolgerung zu, daß sich die untersuchte Elektron-Loch-Plasma-Expansion in GaAs bei Raumtemperatur mit einem hydrodynamischen Diffusionsmodell mit $D=D(n(z))$ mit dem Fermidruck als zugrundeliegender treibender Kraft beschreiben läßt (siehe auch Diskussion in Kap.7). 


\section{Kapitel 6}

\section{EHP-Expansion bei $T_{L}<300 K$}

Nachdem im vorherigen Kapitel die Elektron-Loch-Plasma-Expansion bei Raumtemperatur untersucht wurde, soll in diesem Kapitel der Frage nachgegangen werden, ob die schnelle und dichteabhängige Plasmaexpansion auch bei tiefen Temperaturen zu beobachten ist. Insbesondere soll untersucht werden, ob und wie die starke Abhängigkeit der Elektron- und Lochbeweglichkeiten von der Temperatur [Mad64 den Hochdichte-Transport beeinflußt. Um dies zu untersuchen, wurden folgende Flugzeitmessungen durchgeführt:

- Variation der Gittertemperatur bei fester Fluenz $F=800 \mu \mathrm{Jcm}^{-2}$ und Probendicke $d=1,1 \mu \mathrm{m}$,

- Variation der Probendicke $d$ bei einer Temperatur von $T_{L}=7 \mathrm{~K}$ bei einer Fluenz von $F=800 \mu \mathrm{Jcm}^{-2}$

- Variation der Pumpfluenz bei $T_{L}=4 \mathrm{~K}$ und $d=1,4 \mu \mathrm{m}$

Diese Messungen werden im ersten Abschnitt vorgestellt. Danach wird im zweiten Abschnitt das im vorherigen Kapitel vorgestellte hydrodynamische Diffusionsmodell erweitert, um im dritten Abschnitt einen Vergleich der Modellsimulationen mit den experimentellen Ergebnissen durchzuführen.

\subsection{Experimentelle Untersuchungen}

\subsubsection{Variation der Gittertemperatur}

In einer ersten Untersuchung sollen Flugzeitmessungen bei konstanter Probendicke und Pumpfluenz durchgeführt werden. Für diese Messung ist jedoch zu beachten, daß die Bandlücke $E_{G}$ von GaAs mit abnehmender Gittertemperatur von $E_{G}\left(T_{L}=300 \mathrm{~K}\right)=$ $1,428 \mathrm{eV}$ auf $E_{G}\left(T_{L}=2 \mathrm{~K}\right)=1,519 \mathrm{eV}$ zunimmt (siehe Abb. 2.2). Diese Tatsache ist bei der Wahl der Probephotonenenergie $E_{\text {probe }}$ in der Nähe des soh-c Übergangs $E_{\text {probe }} \sim E_{G}+\Delta_{\text {soh }}$ und bei der Bestimmung der jeweiligen Probendicke $d$ über die Transmission der Probepulse zu beachten. Da eine Variation von $E_{\text {probe }}$ innerhalb einer Meßreihe aufgrund der damit 
verbundenen Nachjustagen nicht sinnvoll ist, wurde eine feste Probephotonenenergie von $E_{\text {probe }}=1,906$ gewählt, welche bei $T_{L}=300 \mathrm{~K} 138 \mathrm{meV}$ und bei $T_{L}=4 \mathrm{~K} 48 \mathrm{meV}$ oberhalb des soh-c Übergangs liegt. In einer Voruntersuchung konnte gezeigt werden, daß sich die $\Delta R_{\text {trans }}(\tau)$ Signale bei einer Variation von $E_{\text {probe }}$ bei konstanter Temperatur (300K) innerhalb von $E_{G}+\Delta_{\text {soh }}+40 \mathrm{meV} \leq E_{\text {probe }} \leq E_{G}+\Delta_{\text {soh }}+150 \mathrm{meV}$ nur in der Intensität aber nicht in ihrem Verlauf wesentlich unterscheiden [Wit99.

Die Probendicke $d$ wurde mit den optischen Konstanten von GaAs bei der jeweiligen Temperatur bestimmt und konnte mit einer Genauigkeit von $\pm 0,1 \mu \mathrm{m}$ auf $d=1,1 \mu \mathrm{m}$ konstant gehalten werden ${ }^{1}$.

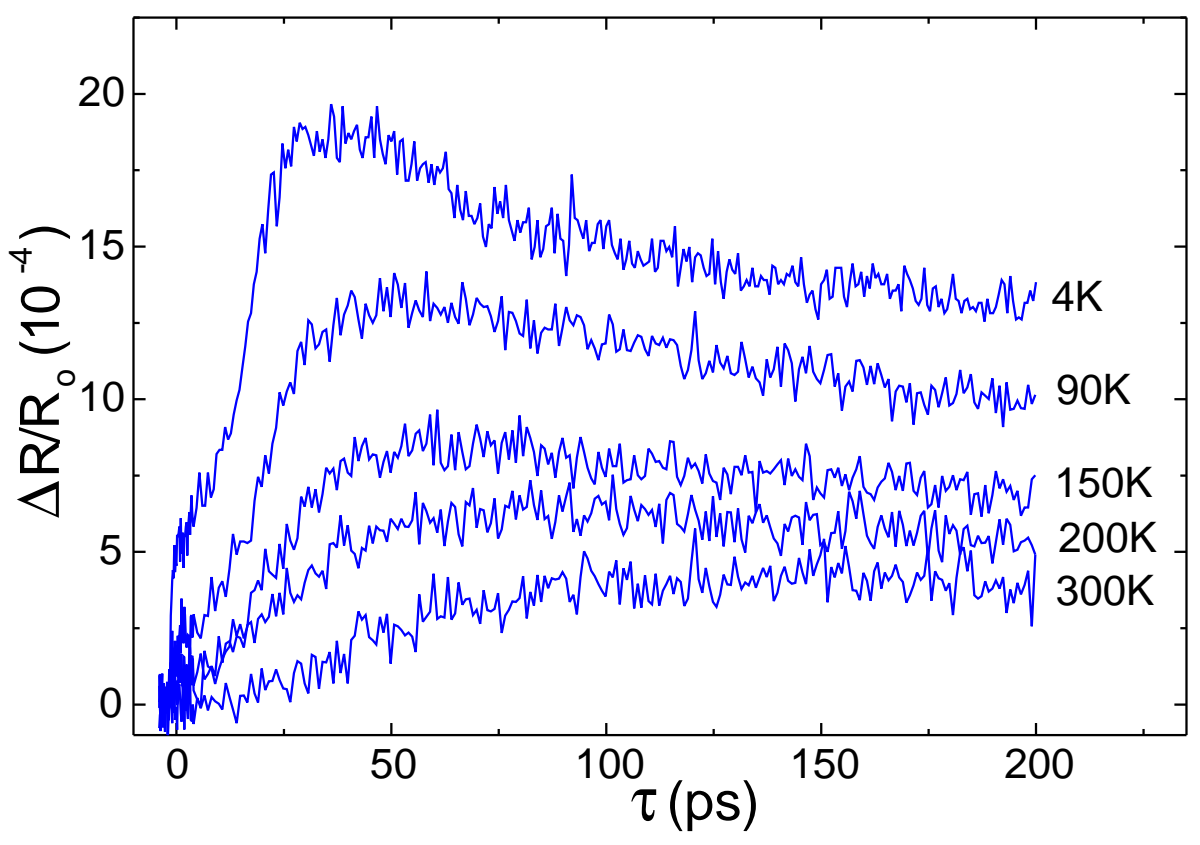

Abbildung 6.1: $\Delta R_{\text {trans }}(\tau)$ : Variation der Gittertemperatur bei $d=1,1 \mu \mathrm{m} ; F=$ $800 \mu \mathrm{Jcm}^{-2} ; E_{\text {pump }}=2,4 \mathrm{eV}$.

In der Abbildung 6.1 sind die $\Delta R_{\text {trans }}(\tau)$ Kurven bei Variation der Gittertemperatur dargestellt. Man erkennt, daß mit abnehmender Temperatur der Anstieg der $\Delta R_{\text {trans }}(\tau)$ Signale zu früheren Verzögerungszeiten $\tau$ verschoben wird.

Somit findet bei $F=800 \mu \mathrm{Jcm}^{-2}$ mit abnehmender Gittertemperatur ein schnellerer Plasmatransport statt.

Dieses Resultat ist erstaunlich, da für Temperaturen $<70 \mathrm{~K}$ die Niedrigdichte-

\footnotetext{
${ }^{1} \mathrm{Da}$ bei der benutzten Apparatur mit der Variation der Temperatur aufgrund thermischer Verspannungen eine geringfügige Verschiebung der lateralen Position der Pump-und Probestrahlfoki auf der Probe auftrat, mußte diese durch eine jeweils neue Positionierung der Probe ausgeglichen werden.
} 
Diffusionskoeffizienten der Elektronen und Löcher aufgrund der ionisierten Störstellenstreuung abnehmen und somit ein langsamerer Transport zu erwarten wäre.

\subsection{2 $T_{L}=7 \mathrm{~K}$ : Variation der Probendicke}

In der Abbildung 6.2 sind die $\Delta R_{\text {trans }}(\tau)$ Kurven bei der Variation der Probendicke $d$ dargestellt. Wie erwartet, wird auch hier ein schnellerer Anstieg des $\Delta R_{\text {trans }}(\tau)$ Signals mit abnehmender Probendicke $d$ gefunden. Die aus den $\Delta R_{\text {trans }}(\tau)$ Kurven aus Abb.

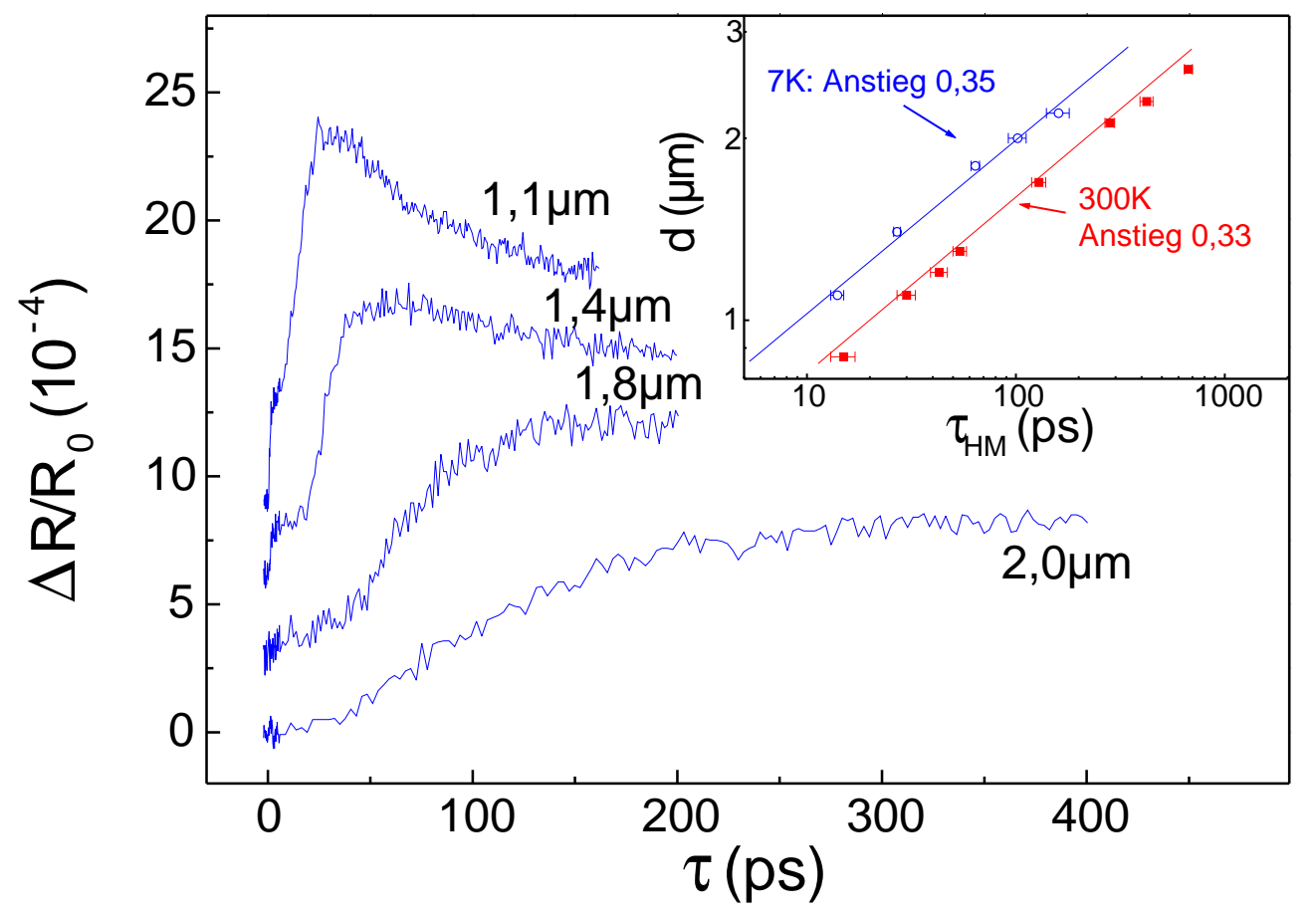

Abbildung 6.2: $\Delta R_{\text {trans }}(\tau)$ : Variation der Probendicke $d$ bei $T_{L}=7 \mathrm{~K} ; F=800 \mu \mathrm{Jcm}^{-2}$; $E_{\text {pump }}=2,4 \mathrm{eV}$. Inset: Ankunftszeiten der Ladungsträger $\tau_{H M}$ in Abhängigkeit von der Probendicke $d$ für $T_{L}=300 \mathrm{~K}$ und $T_{L}=7 \mathrm{~K}$

6.2 bestimmten Ankunftszeiten der Ladungsträger sind im Inset auf einer doppellogarithmischen Skala aufgetragen. Um einen direkten Vergleich zu ermöglichen, sind in der Abbildung zusätzlich die bei Raumtemperatur unter identischen Anregungsbedingungen (Pumpfluenz, Pumpphotonenenergie) bestimmten Werte dargestellt.

Man erkennt zum einen, daß bei $T_{L}=7 \mathrm{~K}$ im Vergleich $z u T_{L}=300 \mathrm{~K}$ ein schnellerer Transport stattfindet, und zum anderen, daß bei beiden Temperaturen ein vergleichbarer Zusammenhang zwischen der Ankunftszeit der Ladungsträger $\tau_{H M}$ und der Probendicke $d$ besteht:

$$
T_{L}=7 K: d \propto \tau_{H M}^{0,35} T_{L}=300 K: d \propto \tau_{H M}^{0,33}
$$




\subsection{3 $T_{L}=4 \mathrm{~K}$ : Variation der Pumpfluenz}

In der Abbildung 6.3 ist die Abhängigkeit der $\Delta R_{\text {trans }}(\tau)$ Signale von der Pumpfluenz $F$ bei einer Temperatur von $T_{L}=4 K$ und einer Probendicke von $d=1,4 \mu \mathrm{m}$ dargestellt. Man erkennt, daß wie bei $T_{L}=300 \mathrm{~K}$ auch bei $T_{L}=4 K$ die $\Delta R_{\text {trans }}(\tau)$ Signale stark von der Pumpfluenz abhängig sind.

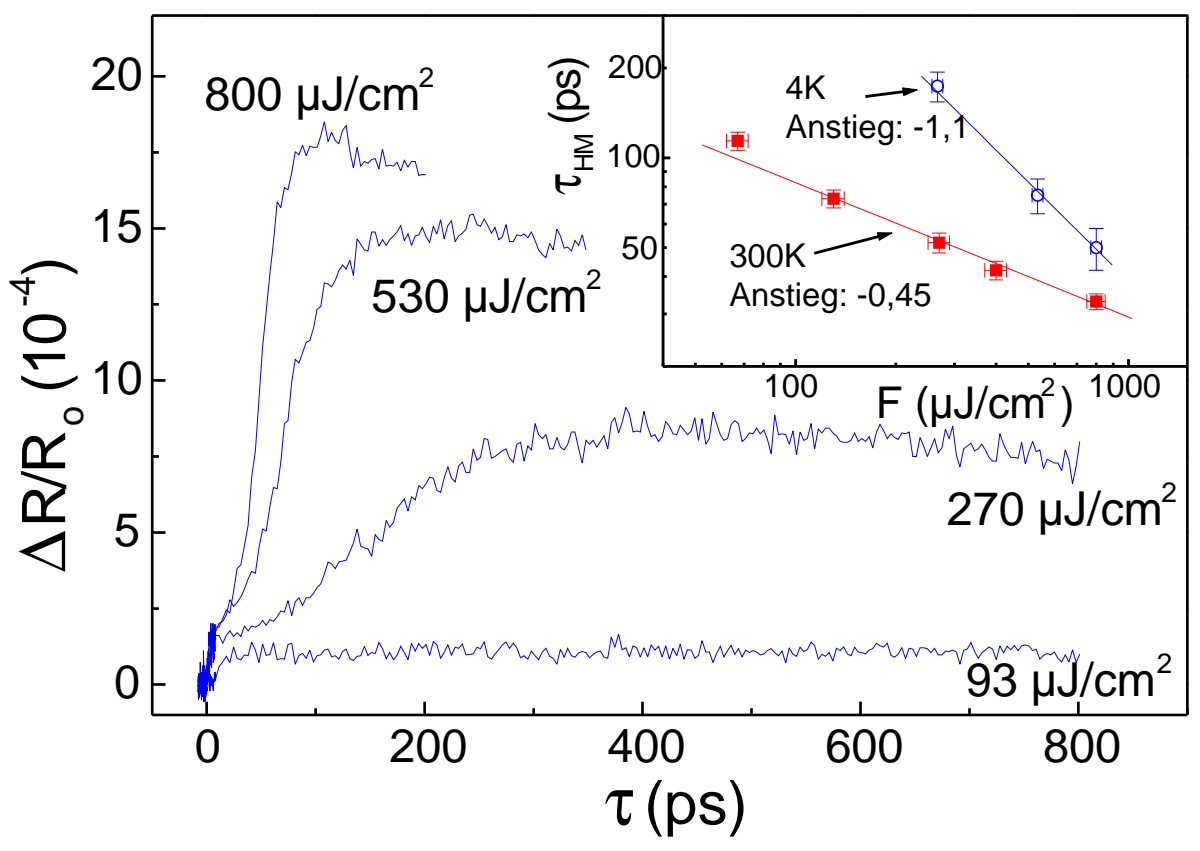

Abbildung 6.3: $\Delta R_{\text {trans }}(\tau)$ : Variation der Pumpfluenz $F$ bei $T_{L}=4 \mathrm{~K} ; d=1,4 \mu \mathrm{m}$; $E_{\text {pump }}=2,4 \mathrm{eV}$. Im Inset sind Ankunftszeiten der Ladungsträger $\tau_{H M}$ in Abhängigkeit von der Pumplluenz $F$ für $T_{L}=300 \mathrm{~K}(d=1,4 \mu \mathrm{m})$ und $T_{L}=4 \mathrm{~K}(d=1,1 \mu \mathrm{m})$ aufgetragen.

Die aus den $\Delta R_{\text {trans }}(\tau)$ Signalen von Abb. 6.3 bestimmten Ankunftszeiten der Ladungsträger $\tau_{H M}$ als Funktion der Fluenz $F$ sind zusammen mit den bei $T_{L}=300 \mathrm{~K}$ gemessenen Werten im Inset von Abbildung 6.3 aufgetragen.

Der Abbildung 6.3 kann man entnehmen, daß die Ankunftszeit der Ladungsträger $\tau_{H M}$ bei $T_{L}=4 \mathrm{~K}$ viel stärker von der Fluenz $F$ abhängig ist als bei $T_{L}=300 \mathrm{~K}$ :

$$
T_{L}=4 K: \tau_{H M} \propto 1 / F^{1,1} T_{L}=300 K: \tau_{H M} \propto 1 / F^{0,45}
$$

Somit wird im Vergleich zu $T_{L}=300 \mathrm{~K}$ bei tiefen Temperaturen $\left(T_{L}=4 K\right)$ eine wesentlich stärkere Abhängigkeit der Geschwindigkeit der Elektron-Loch-Plasma-Expansion von der Anregungsfluenz F gefunden. 
Die im Vergleich zu $T_{L}=300 \mathrm{~K}$ größeren Werte für $\tau_{H M}$ kommen dadurch zustande, daß bei $T_{L}=4 \mathrm{~K}$ bei einer größeren Probendicke gemessen wurde $(4 \mathrm{~K}: d=1,4 \mu \mathrm{m} ; 300 \mathrm{~K}: d=$ $1,1 \mu \mathrm{m})$. Die unterschiedliche Probendicke hat jedoch keinen Einfluß auf die Korrelation von $\tau_{H M}$ und $F$.

\section{Zusammenfassung der experimentellen Ergebnisse für $T_{L}<300 \mathrm{~K}$}

Die Untersuchung der Elektron-Loch-Plasma-Expansion bei Gittertemperaturen $T_{L}<$ 300K hat folgende drei wesentliche Ergebnisse geliefert:

- Mit abnehmender Gittertemperatur wurde in dem Bereich von $300 \mathrm{~K} \geq T_{L} \geq 4 \mathrm{~K}$ bei einer Fluenz von $F=800 \mu \mathrm{Jcm}^{-2}$ eine schnellere Elektron-Loch-Plasma-Expansion in GaAs gefunden.

- Während sich die Absolutwerte der Expansionsgeschwindigkeit bei der Variation der Gittertemperatur unterscheiden, ergibt sich für $T_{L}=300 \mathrm{~K}$ und $T_{L}=7 \mathrm{~K}$ ein vergleichbarer funktionaler Zusammenhang zwischen der Expansionsgeschwindigkeit $v$ und der Verzögerungszeit $\tau\left(300 \mathrm{~K} v \propto \tau^{-0,67} ; 7 \mathrm{~K}: v \propto \tau^{-0,65}\right)$.

- Bei tiefen Temperaturen $\left(T_{L}=4 \mathrm{~K}\right)$ ist die Geschwindigkeit der Plasmaexpansion im Vergleich zu $T_{L}=300 \mathrm{~K}$ wesentlich stärker von der Anregungsfluenz $F$ abhängig. Die Ankunftszeit der Ladungsträger $\tau_{H M}$ bei konstanter Probendicke ist wie folgt von der Fluenz $F$ abhängig: $\tau_{H M} \propto 1 / F^{1,1}(4 \mathrm{~K}) ; \tau_{H M} \propto 1 / F^{0,45}(300 \mathrm{~K})$. 


\subsection{Theoretische Beschreibung des Plasmatranspor- tes für $T_{L}<300 \mathrm{~K}$}

In diesem Abschnitt soll der Frage nach der Ursache der schnellen Plasmaexpansion in GaAs bei tiefen Temperaturen $T_{L}$ nachgegangen werden.

In einem ersten Versuch würde man die Plasmaexpansion bei tiefen Temperaturen $T_{L}$ mit dem in Kap. 5.2.1 vorgestellten hydrodynamischen Diffusionsmodelles beschreiben. Dort war angenommen worden, daß die Ladungsträgertemperatur $T_{c}$ der Gittertemperatur $T_{L}$ entspricht: $T_{c}=T_{L}$. Eine Variation der Temperatur würde eine Änderung des dichteabhängigen Diffusionskoeffizienten $D=D(n)$ (Gl. (5.14)) zur Folge haben. Als Vorfaktor tritt dort die ambipolare Niedrigdichte-Diffusivität $D_{a m b}^{0}=\left(2 D_{e}^{0} D_{h}^{0}\right) /\left(D_{e}^{0}+D_{h}^{0}\right)$ auf. Wobei die Niedrigdichte-Diffusivitäten $D^{0}$ der Einsteinrelation: $D_{c}^{0}=\left(k_{b} T_{c} \mu_{c}^{0}\right) / e$ genügen .

(a)

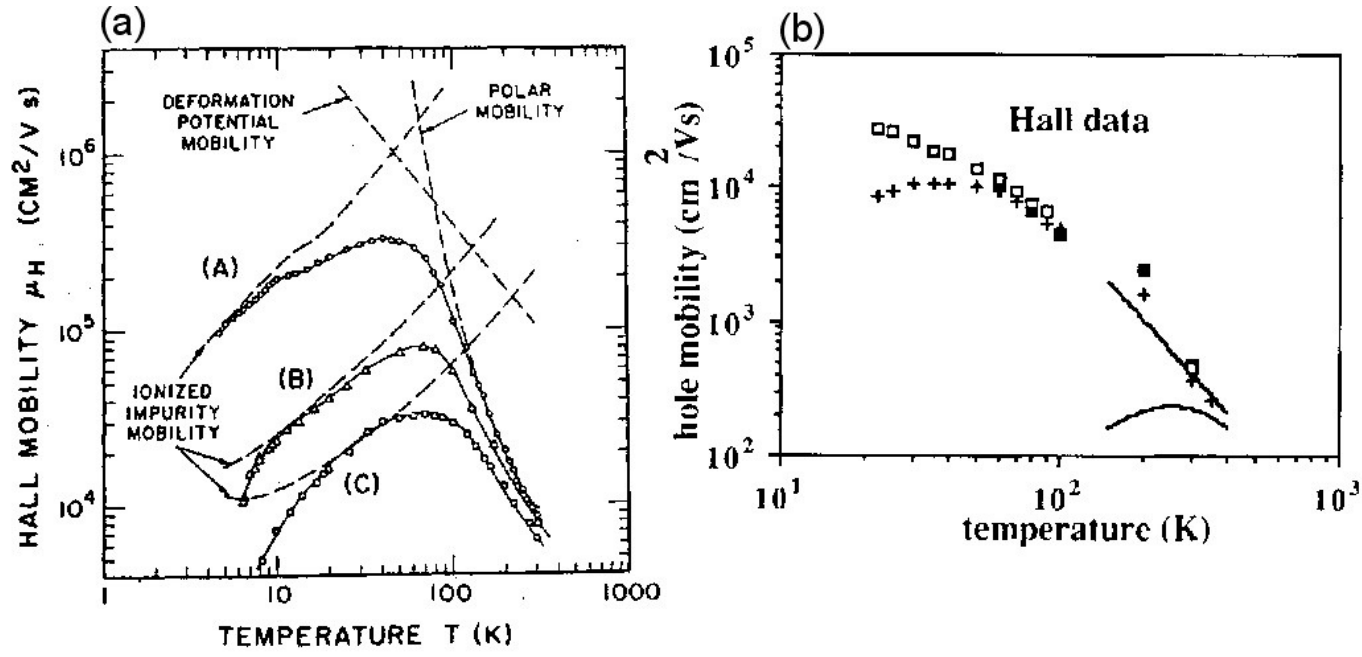

Abbildung 6.4: (a) Temperaturabhängigkeit der Elektronen-Hallbeweglichkeit $(B=$ $5 \mathrm{kG}$ ) für N-Typ GaAs Proben mit einer abgeschätzten Donatorkonzentration $N_{D}:(\mathrm{A})$ $5 \times 10^{13} \mathrm{~cm}^{-} 3$; (B) $10^{15} \mathrm{~cm}^{-} 3$ und (C) $5 \times 10^{15} \mathrm{~cm}^{-} 3$. Als gestrichelte Linien sind die theoretisch berechneten Beiträge der drei wesentlichen Streuprozesse zu $\mu_{e}$ dargestellt [Sti70]. (b) Temperaturabhängigkeit der Löcherbeweglichkeit in GaAs. Experimentelle Daten nach [Hil70, Mea71, Zsc73]. Theoretische Kurven nach [Shu87].

Die Abhängigkeit der Beweglichkeiten $\mu_{c}^{0}$ der Elektronen und Löcher von der Temperatur ist in der Abbildung 6.4 dargestellt. Die Beweglichkeiten wurden dort mit Hilfe des Hall Effektes bestimmt ${ }^{2}$, wobei die Ladungsträgerkonzentration der Elektronen oder Löcher durch Dotierung des Materials mit einer Donator- bzw. Akzeptorkonzentration

\footnotetext{
${ }^{2}$ Die Hallbeweglichkeit $\mu_{\text {hall }}$ unterscheidet sich von der Beweglichkeit $\mu_{c}$ durch den Hallfaktor $r_{H}$ $: \mu_{\text {hall }}=\mu_{c} r_{H}$, welcher bei hohen B-Felder einen Wert Nahe eins besitzt (siehe z.B. [See92]).
} 
$N_{D}$ bzw. $N_{A}$ erzielt wurde. Variiert wurde die Gittertemperatur $T_{L}$, welche bei diesen Messungen, die nahe am thermischen Gleichgewicht durchgeführt wurden, auch der Ladungsträgertemperatur $T_{c}$ entspricht.

Man erkennt in Abbildung 6.4, daß die Beweglichkeit sowohl der Elektronen als auch der Löcher ab ca. 70K mit abnehmender Temperatur aufgrund der ionisierten StörstellenStreuung abnimmt. Somit nehmen auch die Niedrigdichte-Diffusivitäten in diesem Temperaturbereich ab.

Setzt man diese temperaturabhängigen Beweglichkeiten bzw. Niedrigdichte-Diffusivitäten in den Ausdruck für die dichteabhängige Diffusivität ein, ergibt die Simulation eine wesentliche Abnahme der Geschwindigkeit der Elektron-Loch-Plasma-Expansion für Temperaturen $T<70 K$. Somit kann das hydrodynamische isothermische Diffusionsmodell aus Kap. 5 mit $T_{c}=T_{L}$ die gefundenen hohen Expansionsgeschwindigkeiten bei tiefen Temperaturen $T_{L}<10 K$ nicht beschreiben.

Eine wesentliche Ursache für das Versagen des isothermischen Modelles für die Beschreibung der Experimente bei $T_{L}<300 \mathrm{k}$, ist die Tatsache, daß die Annahme: $T_{c}=T_{L}$ bei tiefen Temperaturen nicht mehr gerechtfertigt ist. Wie in Kap. 2.2 dargelegt wurde, ist die Abkühlung der ursprünglich heißen Ladungsträger auf eine Temperatur $T_{c}=300 \mathrm{~K}$ nach ca. 10ps abgeschlossen, eine weitere Abkühlung auf $T_{c} \approx 4 \mathrm{~K}$ findet dagegen auf einer Zeitskala von $\sim 100 \mathrm{ps}$ statt. Dies bedeutet, daß die Annahme $T_{c}=T_{L}$ zwar für $T_{L}=300 \mathrm{~K}$ gerechtfertigt ist, da hier der untersuchte Plasmatransport auf einer Zeitskala stattfindet, die größer ist als die der Ladungsträgerabkühlung. Für die Beschreibung der Plasmaexpansion bei Gittertemperaturen $T_{L}<300 \mathrm{~K}$ muß die Ladungsträgerabkühlung jedoch berücksichtigt werden.

\section{Erweiterung des Hydrodynamischen Diffusionsmodelles auf $T_{c}=T_{c}(\tau)$}

In welcher Form die Ladungsträgerkühlung in dem hydrodynamischen Diffusionsmodell aus Kap. 5.2.1 berücksichtigt werden kann, ist Thema dieses Abschnittes. Wie in Kap. 5.2.1 bereits dargelegt, ist eine adäquate Beschreibung des Transportes von optisch mit hohen kinetischen Energien angeregten Ladungsträgern unter Berücksichtigung der wesentlichen Streumechanismen nur mit Molekular-Dynamik bzw. Monte-CarloSimulationen möglich. An dieser Stelle soll die Ladungsträgerkühlung jedoch nur in der einfachsten nichttrivialen Näherung in dem hydrodynamischen Diffusionsmodell berücksichtigt werden. Dieses geschieht dadurch, daß die Ladungsträgertemperatur $T_{c}$ als eine Funktion der Verzögerungszeit $\tau$ beschrieben wird: $T_{c}=T_{c}(\tau)$. Für die Simulation wird der zeitliche Verlauf der Ladungsträgertemperatur $T_{c}(\tau)$ der Literatur entnommen (Kurve mit $\mathrm{C}=0,1$ aus Abb. 2.6 in Kap. 2.2). Der Einfachheit halber wird hierbei die Abhängigkeit der Ladungsträgerkühlung von der Anregungsdichte vernachlässigt ${ }^{3}$.

\footnotetext{
${ }^{3}$ I. Allg. ist der zeitliche Verlauf der Ladungsträgertemperatur aufgrund von Abschirmungseffekten bzw. heißer Phononen Effekte von der Anregungsdichte abhängig. Für die in dieser Arbeit verwendeten hohen Anregungsdichten von $n_{0}>10^{19} \mathrm{~cm}^{-3}$ ist dieser Zusammenhang nur sehr ungenau bekannt [Sha99] und wird deshalb hier vernachlässigt.
} 
Die von der Verzögerungszeit $\tau$ abhängige Ladungsträgertemperatur wird nun in dem Ausdruck für den dichteabhängigen Diffusionskoeffizienten $D=D(n)$ in Gl. (5.14) in der Form von $D=D\left(n, T_{c}(\tau)\right)$ berücksichtigt:

$$
D=\frac{1}{q^{2}} \frac{1}{n \beta} \frac{\sigma_{e} \sigma_{h}}{\sigma_{e}+\sigma_{h}}\left[\frac{\mathcal{F}_{1 / 2}\left(\beta \psi_{h}\right)}{\mathcal{F}_{-1 / 2}\left(\beta \psi_{h}\right)}+\frac{\mathcal{F}_{1 / 2}\left(\beta \psi_{e}\right)}{\mathcal{F}_{-1 / 2}\left(\beta \psi_{e}\right)}\right] \quad \text { mit: } \quad \sigma_{c}=e n_{c} \mu_{c}^{0} \frac{\mathcal{F}_{0}\left(\beta \psi_{c}\right)}{\mathcal{F}_{1 / 2}\left(\beta \psi_{c}\right)} .
$$

Bei einer Änderung der Ladungsträgertemperatur ergeben sich zum einen Änderungen in den Abhängigkeiten der Fermiintegrale $\mathcal{F}_{j}(\beta \psi)$ von der Dichte $n$ und zum anderen sind auch die Niedrigdichte-Beweglichkeiten $\mu_{c}^{0}$ von der Temperatur abhängig.

Der Einfluß der Fermiintegrale auf den dichteabhängigen Diffusionskoeffizienten bei Variation der Ladungsträgertemperatur $T_{c}$ ist in der Abbildung 6.5 dargestellt. Hierbei wurden nur die Fermiintegrale für die unterschiedlichen Ladungsträgertemperaturen $T_{c}$ neu berechnet. Alle anderen Parameter wurden konstant gehalten. Man erkennt, daß eine Verringerung der Temperatur $T_{c}$ lediglich eine Verschiebung der $D(n)$ Kurve zu niederen Dichten hin zur Folge hat. Dieses Verhalten ist darauf zurückzuführen, daß bei tieferen Ladungsträgertemperaturen der Übergang von dem nicht entarteten zum entarteten Fermigas schon bei einer geringeren Dichte $n$ stattfindet.

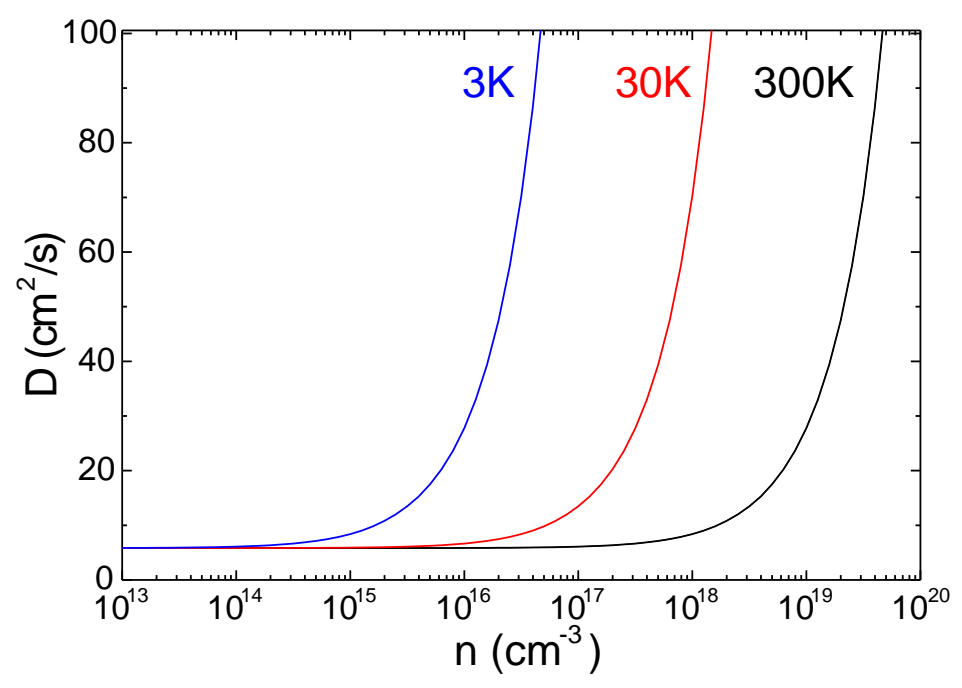

Abbildung 6.5: $\mathrm{D}(\mathrm{n})$ bei Variation der Ladungsträgertemperatur $T_{c}$ : nur berücksichtigt in den Fermiintegralen

Die temperaturabhängigen Niedrigdichte-Beweglichkeiten $\mu_{h}\left(T_{c}\right)$ von Elektronen und Löchern sind von der Dichte der ionisierten Störstellen in der jeweiligen Probe abhängig (siehe Abb. 6.4). Für die in den Experimenten verwendeten kompensiert dotierten Proben kann diese jedoch nur grob auf $10^{15} \mathrm{~cm}^{-3}<N_{i}<10^{16} \mathrm{~cm}^{-3}$ abgeschätzt werden 4 . Für die

\footnotetext{
${ }^{4}$ Diese Abschätzung von $N_{i}$ erfolgte anhand der Linienbreite des 1s Exzitons im Absorptionsspektrum.
} 
Simulationen wurde eine Dichte von $N_{i}=5 \times 10^{15} \mathrm{~cm}^{-3}$ benutzt.

Eine weitere Schwierigkeit besteht darin, daß für die Simulation die Abhängigkeit der Ladungsträgerbeweglichkeiten von der Ladungsträgertemperatur $T_{c}$ bei der jeweils verwendeten konstanten Gittertemperatur $T_{L}$ benötigt würde. Experimentell können mit Hilfe des Hall Effektes nur Beweglichkeiten bei $T_{c}=T_{L}$ bestimmt werden. Eine theoretische Bestimmung der Beweglichkeiten ist mit Hilfe von Monte Carlo Simulationen als Lösung der Boltzmanngleichung unter den jeweiligen Bedingungen möglich [Jac89, Shu87, aber im Rahmen des hier verwendeten hydrodynamischen Diffusionsmodelles nicht sinnvoll. Als grobe Näherung werden daher hier die aus Hall Messungen bestimmten Beweglichkeiten $\mu_{c}(T)$ für die Abhängigkeit der Beweglichkeit $\mu_{c}$ von der Ladungsträgertemperatur $T_{c}$ für alle Gittertemperaturen $T_{L}$ eingesetzt. Als analytischen Ausdruck für die Temperaturabhängigkeit der Beweglichkeit wird hier der in [Shu87] angegebene verwendet.

Die so erhaltene dichte- und ladungsträgertempereraturabhängige Diffusivität $D\left(n, T_{c}\right)$ ist in der Abbildung 6.6 auf einer logarithmischen Skala dargestellt. Man erkennt, daß die

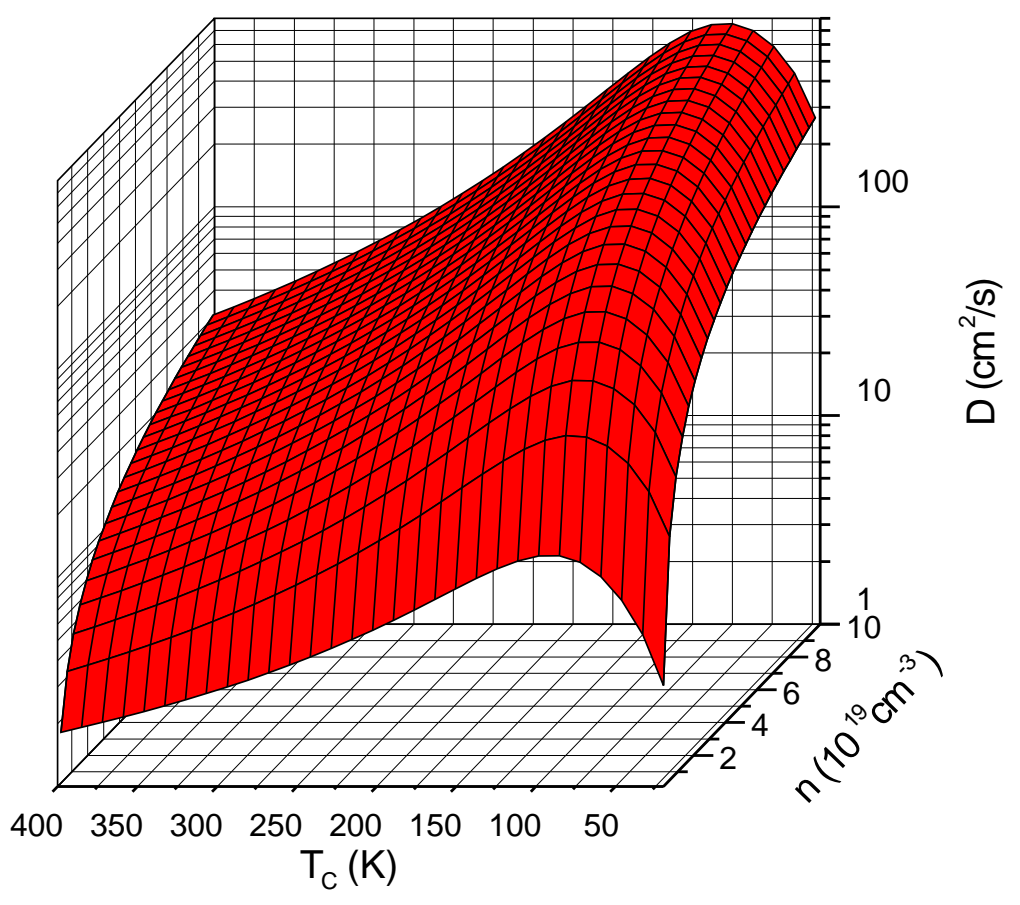

Abbildung 6.6: Diffusionskoeffizient $D\left(n, T_{c}\right)$ als Funktion der Ladungsträgertemperatur $T_{c}$ und Dichte $n$

so bestimmte Diffusivität bei konstanter Dichte jeweils ein Maximum bei einer Temperatur $T_{c} \sim 100 \mathrm{~K}$ besitzt und zu tiefen Temperaturen steil abfällt. Dieses Verhalten spiegelt den Einfluß der Niedrigdichte-Diffusivitäten $D_{c}^{0}$ wider, die über die Einsteinrelation mit

Die Absorptionsmessung wurde an einer Probe (Probe \#1 in Dre93]) des Wafers durchgeführt, aus dem auch die in dieser Arbeit verwendeten Proben präpariert wurden [Dre93. 
Kapitel 6 EHP-Expansion bei $T_{L}<300 K$

den Beweglichkeiten $\mu_{c}$ verknüpft sind. Bei einer konstanten Temperatur $T_{c}$ steigt die Diffusivität mit zunehmender Dichte $n$ aufgrund des Fermidruckes an, und zwar bei tiefen Temperaturen $T_{c}$ stärker als bei hohen Temperaturen $T_{c}$. Verantwortlich hierfür ist die Tatsache, daß bei tiefen Temperaturen $T_{c}$ die Entartung und damit auch der Einfluß des Fermidruckes schon bei geringeren Dichten $n$ einsetzt als bei höheren Temperaturen $T_{c}$ (siehe Abb. 6.5).

Mit diesem dichte- und temperaturabhängigen Diffusionskoeffizienten $D\left(n, T_{c}\right)$ in Verbindung mit der Zeitabhängigkeit der Ladungsträgertemperatur $T_{c}=T_{c}(\tau)$ wurde die Diffusionsgleichung mit den in Kap. 5.2 vorgestellten Anfangs- und Randbedingungen numerisch gelöst. Auch für die Koeffizienten der Auger- und Plasmarekombination wurden die identischen Werte von Kap. 5.2 eingesetzt. Die Ergebnisse der Simulation werden im folgenden Abschnitt im Vergleich mit den experimentellen Kurven diskutiert.

\subsection{Vergleich Experiment - Simulation}

In diesem Abschnitt werden die Flugzeitmessungen bei Gittertemperaturen $T<300 \mathrm{~K}$ (siehe Kap. 6.1) mit Simulationsrechnungen basierend auf dem im vorherigen Abschnitt vorgestellten hydrodynamischen Diffusionsmodell mit einem Diffusionskoeffizienten $D=$ $D\left(n, T_{c}(\tau)\right)$ verglichen.

\subsubsection{Vergleich Experiment - Simulation: Variation der Gitter- temperatur}

In der Abbildung 6.7 sind die $\Delta R_{\text {trans }}(\tau)$ Kurven und die aus der Simulation bestimmten zeitlichen Verläufe der Dichte $n$ bei $z=d$ dargestellt. Die absolute Höhe der $n(\tau, z=d)$ Kurven ist für einen besseren Vergleich mit dem Experiment den jeweiligen Meßkurven angepaßt.

Man erkennt, daß die Simulation den wesentlichen Trend der Messungen: Erhöhung der Expansionsgeschwindigkeit bei abnehmender Gittertemperatur reproduziert. Die Tatsache, daß die Expansionsgeschwindigkeit für Temperaturen $T_{L}<100 \mathrm{~K}$ in der Simulation nicht abnimmt, wird dadurch verursacht, daß die Ladungsträgertemperatur erst für Verzögerungszeiten $\tau>10 \mathrm{ps}$ auf unter 100K abgefallen ist. Bei der hier untersuchten Probendicke von $d=1,1 \mu \mathrm{m}$ haben die Ladungsträger jedoch schon nach ca. 15ps die gegenüberliegende Probenseite erreicht. Dadurch hat die Abnahme des Diffusionskoeffizienten für $T_{c}<100 \mathrm{~K}$ (siehe Abb. 6.6) nur einen sehr geringen Einfluß auf die hier untersuchte Plasmaexpansion.

Die gefundene schnelle Elektron-Loch-Plasma-Expansion für Gittertemperaturen $T_{L}<100 K$ kann somit mit dem Transport heißer Ladungsträger $\left(T_{c}>T_{L}\right)$ interpretiert werden. 

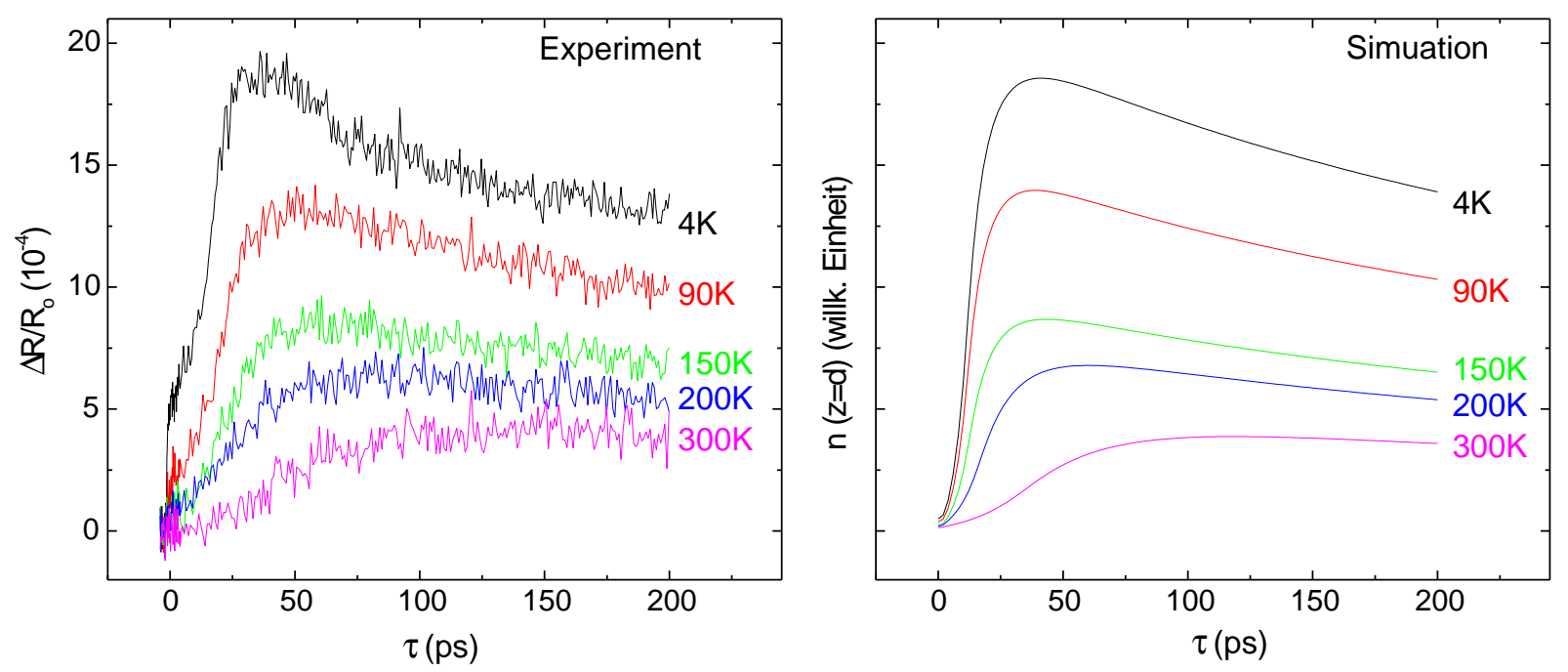

Abbildung 6.7: Vergleich Experiment mit Simulation bei Variation der Gittertemperatur $\left(d=1,1 \mu \mathrm{m} ; F=800 \mu \mathrm{Jcm}^{-2} ; E_{\text {pump }}=2,4 \mathrm{eV}\right.$. $)$

Wird von der Simulation zwar der wesentliche Trend der Messungen wiedergegeben, so ist bei den Temperaturen von $T_{L}=90 \mathrm{~K}, 150 \mathrm{~K}$ und $200 \mathrm{~K}$ ein signifikanter Unterschied in der Ankunftszeit $\tau_{H M}$ zu erkennen. Dieser Unterschied könnte zum einen an den in dem Modell gemachten Näherungen und Unsicherheiten bei der Bestimmung sowohl von $D=D\left(n, T_{c}\right)$ als auch von $T_{c}=T_{c}(\tau)$ liegen (siehe Kap. 6.2). Zum anderen können die Unterschiede auch durch die experimentelle Unsicherheit in der Einstellung der Probendicke von $1,1 \pm 0,1 \mu \mathrm{m}$ (siehe Kap. 6.1.1) verursacht worden sein.

\subsubsection{Vergleich Experiment - Simulation: Variation der Proben- dicke}

In der Abbildung 6.8 sind die $\Delta R_{\text {trans }}(\tau)$ Kurven bei einer Temperatur von $T_{L}=7 \mathrm{~K}$ für unterschiedliche Probendicken $d$ zusammen mit den aus der Simulation bestimmten Kurven dargestellt. Dieser Abbildung kann man entnehmen, daß die Simulationskurven relativ gut mit den Meßkurven übereinstimmen. Insbesondere wird von der Simulation die Abhängigkeit der Ankunftszeit der Ladungsträger $\tau_{H M}$ von der Probendicke (siehe Inset von Abb. 6.8 richtig reproduziert.

Unterschiede im Kurvenverlauf von Experiment und Simulation sind vor allem bei der kleinsten $(d=1,1 \mu \mathrm{m})$ und größten $(d=2,0 \mu \mathrm{m})$ Probendicke festzustellen. Bei $d=1,1 \mu \mathrm{m}$ ist der Abfall des Signals, welcher von der (nichtlinearen) Rekombination verursacht wird, unterschiedlich. Der Unterschied könnte mit einer Temperaturabhängigkeit der Ladungsträgerrekombinationsgeschwindigkeiten erklärt werden, da in der Simulation temperaturunabhängige (sowohl Ladungs- als auch Gittertemperatur) Koeffizienten für 


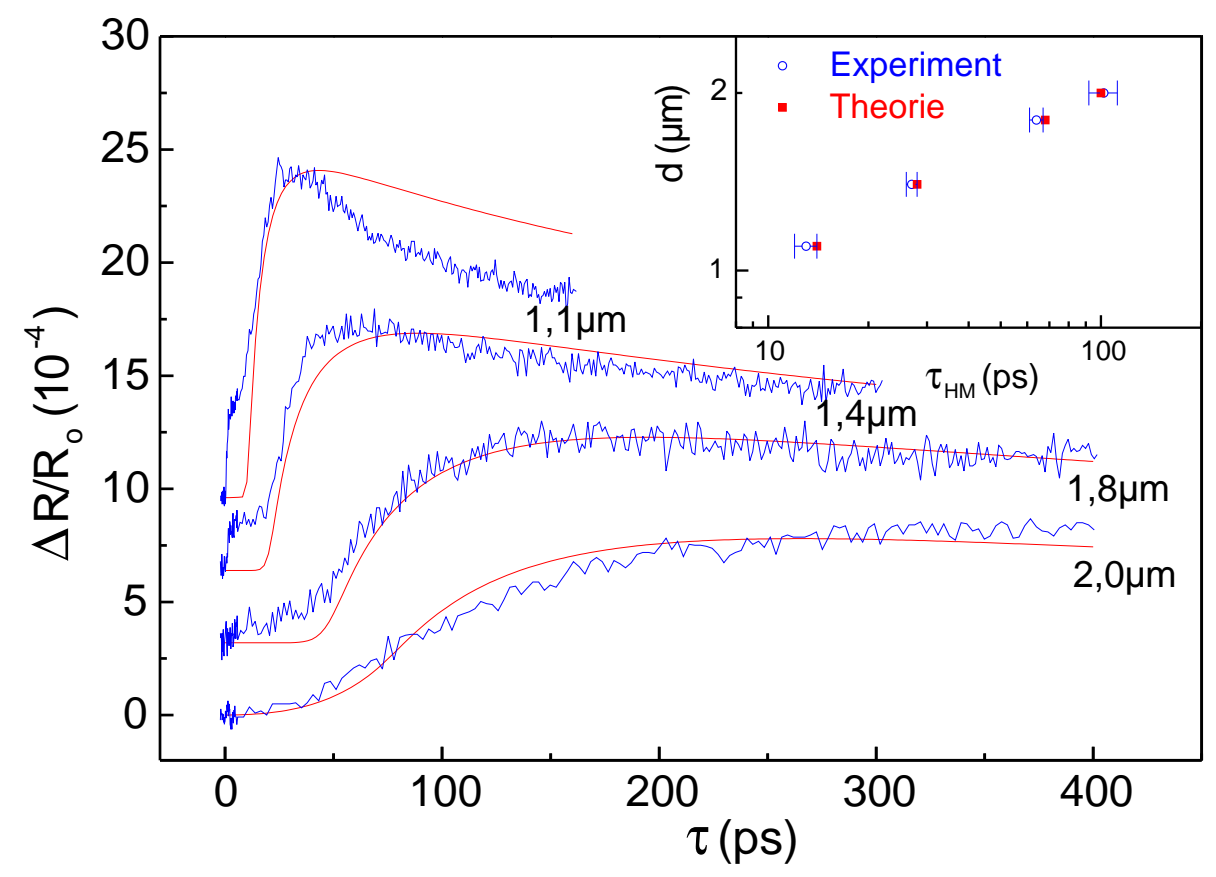

Abbildung 6.8: Vergleich Experiment mit Simulation bei Variation der Probendicke $d$ $\left(T_{L}=7 \mathrm{~K} ; F=800 \mu \mathrm{Jcm}^{-2} ; E_{\text {pump }}=2,4 \mathrm{eV}\right)$. Im Inset ist die Abhängigkeit der Ankunftszeit der Ladungsträger $\tau_{H M}$ von der Probendicke $d$ doppellogarithmisch dargestellt.

die Auger- und Plasmarekombination verwendet wurden. Die Ursache für den im Vergleich zur Simulation langsameren Anstieg des $\Delta R_{\text {trans }}(\tau)$ bei $d=2,0 \mu \mathrm{m}$ ist jedoch ungeklärt.

Im Vergleich von Simulation und Experiment fällt weiterhin auf, daß das $\Delta R_{\text {trans }}(\tau)$ Signal bei kleinen Probendicken einen instantanen Anstieg bei $\tau=0$ aufweist, nicht jedoch die Simulationskurven. Wie in Kap. 4.4.2 dargelegt, ist der instantane Anstieg von $\Delta R_{\text {trans }}(\tau)$ auf die schon innerhalb des Detektionsvolumens von $\Delta R_{\text {trans }}$ angeregten Ladungsträger zurückzuführen. Der Einfachheit halber wird jedoch für den Vergleich von Simulation und Experiment angenommen, daß das $\Delta R_{\text {trans }}(\tau)$ Signal proportional zu der Ladungsträgerdichte $n(z=d)$ an der Oberfläche der Probe ist (siehe Kap. 5.2.2). Aufgrund dieser vereinfachenden Annahme wird der instantane Anstieg des $\Delta R_{\text {trans }}(\tau)$ Signals nicht von der Simulation reproduziert. Dafür wäre eine detaillierte Berücksichtigung der örtlichen Sensitivität des $\Delta R_{\text {trans }}(\tau)$ Signals notwendig.

\subsubsection{Vergleich Experiment - Simulation: Variation der Fluenz}

In der Abbildung 6.9 werden die experimentellen $\Delta R_{\text {trans }}(\tau)$ Kurven bei Variation der Pumpfluenz mit den simulierten Ergebnissen verglichen. Man erkennt, daß die im Vergleich zu den Messungen bei $T_{L}=300 \mathrm{~K}$ stärkere Abhängigkeit der $\Delta R_{\text {trans }}(\tau)$ Kurven von der Pumplluenz $F$ gut von der Simulation wiedergegeben wird. Für den Zusammenhang der 


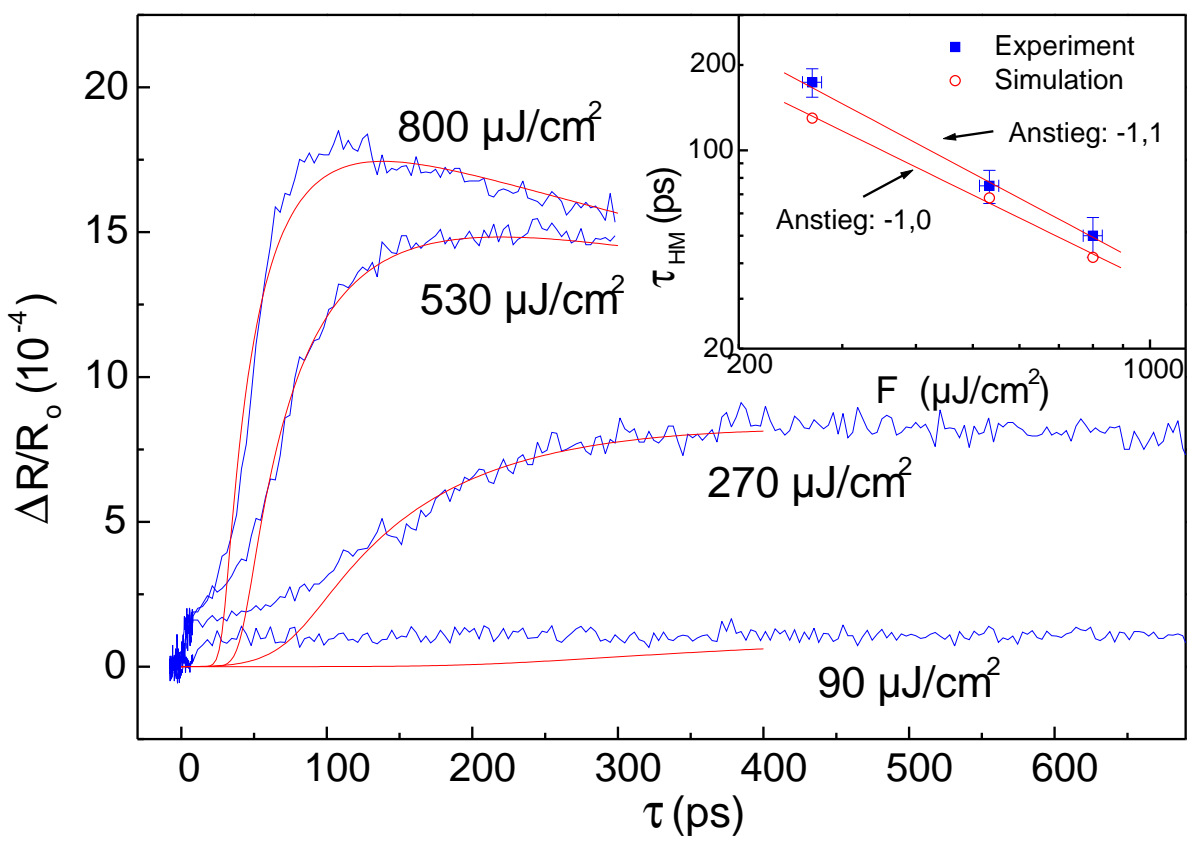

Abbildung 6.9: Vergleich Experiment mit Simulation bei Variation der Pumpfluenz F $\left(T_{L}=4 \mathrm{~K} ; d=1,4 \mu \mathrm{m} ; E_{\text {pump }}=2,4 \mathrm{eV}\right.$.) Im Inset ist die Abhängigkeit der Ankunftszeit der Ladungsträger $\tau_{H M}$ von der Fluenz $F$ auf einer doppellogarithmischen Skala aufgetragen.

Ankunftszeit der Ladungsträger $\tau_{H M}$ von der Pumpfluenz ergibt sich aus der Simulation:

Theorie: $\tau_{H M} \propto 1 / F^{1,0}$

welches relativ gut mit dem aus dem Experiment bestimmten Zusammenhang von: $\tau_{H M} \propto 1 / F^{1,1}$ übereinstimmt.

Die im Vergleich zu $T_{L}=300 \mathrm{~K}$ wesentlich stärkere Dichteabhängigkeit der ElektronLoch-Plasma-Expansion bei tiefen Temperaturen $T_{L}$ kann anhand der Simulationen auf zwei wesentliche Ursachen zurückgeführt werden:

Zum einen ist der Diffusionskoeffizient $D$ bei tiefen Ladungsträgertemperaturen $T_{c}$ stärker von der Ladungsträgerdichte $n$ abhängig (siehe Kap. 6.2), da hier der Übergang zur Entartung schon bei niedrigeren Dichten $n$ einsetzt. Jedoch muß berücksichtigt werden, daß die ursprünglich heißen Ladungsträger sich erst nach einer gewissen Verzögerungszeit $\tau$ auf die tiefen Temperaturen $T_{c}$ abgekühlt haben.

Zum anderen bewirkt die spezielle Zeitabhängigkeit des Diffusionskoeffizienten $D\left(n, T_{c}(\tau)\right)$ eine starke Dichteabhängigkeit. So besitzen die Ladungsträger zu frühen Zeiten bei hohen Dichten einen mittelgroßen Diffusionskoeffizienten, welcher mit zunehmender Verzögerungszeit ansteigt, bis er nach einer gewissen Verzögerungszeit $\tau_{\max }$ 
ein Maximum durchläuft. $\mathrm{Zu}$ diesem Zeitpunkt haben sich die Ladungsträger auf ca. 100K abgekühlt und besitzen immer noch eine mittelgroße Dichte. Danach nimmt der Diffusionskoeffizient deutlich aufgrund der weiteren Ladungsträgerkühlung ab. Dieser zeitliche Verlauf von $D$ mit einem Maximum und einen Abfall zu langen Verzögerungszeiten sorgt dafür, daß der Einfluß der Dichteabhängigkeit des Diffusionskoeffizienten auf die Plasmaexpansion verstärkt wird, indem das Plasma bei einer hohen Dichte die gegenüberliegende Probenseite zu einer Zeit erreicht, bei der es sich noch nicht auf die tiefen Temperaturen $T_{c}$ mit den entsprechend niedrigen Diffusionskoeffizienten abgekühlt hat. Die Plasmaexpansion bei niedrigen Dichten wird dagegen stark von der Abnahme des Diffusionskoeffizienten mit abnehmender Ladungsträgertemperatur $T_{c}$ beeinflußt.

Das Zusammenspiel dieser beiden Faktoren ist für die starke Dichteabhängigkeit der Elektron-Loch-Plasma-Expansion in GaAs bei tiefen Gittertemperaturen $T_{L}$ verantwortlich.

Somit kann die im Vergleich zu $T_{L}=300 \mathrm{~K}$ stärkere Dichteabhängigkeit der ElektronLoch-Plasma-Expansion bei tiefen Temperaturen $T_{L}$ mit dem Transport eines heißen Ladungsträgerplasmas mit $T_{c}>T_{L}$ unter Berücksichtigung der Ladungsträgerkühlung $T_{c}=$ $T_{c}(\tau)$ beschrieben werden. 


\section{Kapitel 7}

\section{Diskussion}

In den Kapiteln 5 und 6 wurden Flugzeitmessungen an optisch generierten Elektron-LochPlasmen in GaAs bei Gittertemperaturen von $T_{L}=300 \mathrm{~K}$ bzw. $T_{L}<300 \mathrm{~K}$ vorgestellt. Die gefundene dichteabhängige Expansion der Ladungsträgerplasmen wurde mit den Resultaten eines einfachen hydrodynamischen Diffusionsmodelles verglichen, welches den Fermidruck als treibende Kraft für die Expansion beinhaltet. Die gute Übereinstimmung der Modellsimulationen mit den experimentellen Ergebnissen legt den Schluß nahe, daß unter den gegebenen experimentellen Bedingungen der Fermidruck die wesentliche Ursache für die schnelle Elektron-Loch-Plasma-Expansion in GaAs ist.

Diese These wird von den von E. Poles durchgeführten Experimenten unterstützt [Pol96, Pol99]. In diesen Experimenten wurde bei $T_{L}=300 \mathrm{~K}$ die zeit- und spektral aufgelöste Lumineszenz in GaAs-Schichten unterschiedlicher Dicke $d$ nach Anregung mit kurzen Pulsen $\left(\tau_{\text {puls }}=2 \mathrm{ps}\right)$ bei einer Photonenenergie $\hbar \omega=2,09 \mathrm{eV}(1 / \alpha \approx 200 \mathrm{~nm})$ gemessen. Die Anregungsdichte betrug $5 \times 10^{18} \mathrm{~cm}^{-3}$. Bei einer Probendicke von $d=5 \mu \mathrm{m}$ wurde ein deutlich schnellerer Abfall der bandkantennahen Lumineszenz gegenüber der bei einer Probendicke von $d=0,5 \mu \mathrm{m}$ gemessenen Lumineszenz gefunden. Dieser Unterschied wird mit dem Einfluß der Fermidruck getriebenen Elektron-Loch-Plasma-Expansion auf die zeitliche Entwicklung der Ladungsträgerdichte $n(z=0, \tau)$ an der Vorderseite der Probe erklärt. Während bei einer Probendicke von $d=0,5 \mu \mathrm{m}$ eine Plasmaexpansion schon nach $\sim 20$ ps abgeschlossen ist und somit danach keinen Einfluß mehr auf die Zeitabhängigkeit der Lumineszenz hat, führt die Elektron-Loch-Plasma-Expansion bei der $d=5 \mu \mathrm{m}$ dicken Probe zu dem untersuchten schnelleren Abfall der Lumineszenz. Diese Meßmethode hat durch die spektrale Auflösung den Vorteil, daß die anhand von Modellvorstellungen getroffenen Aussagen über die zeitliche Entwicklung der Ladungsträgertemperatur $T_{c}(\tau, z=0)$ und die Lage der Quasiferminiveaus $\psi_{c}(\tau, z=0)$ an der Vorderseite der Probe direkt mit den Lumineszenzspektren verglichen werden können. Der Nachteil dieser Methode ist, daß Aussagen über den Transport der Ladungsträger nur indirekt möglich sind, da nur die Ladungsträger an der Probenvorderseite zu dem gemessenen Lumineszenzsignal beitragen. 
Somit ergänzen sich die in der vorliegenden Arbeit durchgeführten Flugzeitmessungen und die von E. Poles [Pol96, Pol99] durchgeführten Messungen der zeitaufgelösten Lumineszenz und legen die Schlußfolgerung nahe, daß der Fermidruck die wesentliche treibende Kraft der Elektron-Loch-Plasma-Expansion in GaAs senkrecht zur Oberfläche ist.

Bevor man jedoch eine solche Schlußfolgerung ziehen kann, müssen die im verwendeten Modell vernachlässigten Effekte, die die Plasmaexpansion jedoch auch beeinflussen können, diskutiert werden.

\section{Bandkantenrenormierung}

Wie in Kap. 2.3 dargelegt, führen Vielteilcheneffekte bei hohen Anregungsdichten $n$ zu einer Renormalisierung der Einteilchenenergien. In der einfachsten Näherung geht man von starren Bändern aus und berücksichtigt nur eine Änderung der Bandlücke $\delta E_{G}$ als Funktion der Ladungsträgerdichte $n: E_{G}(n)=E_{G}^{0}+\delta E_{G}$ (siehe Gl. (2.6)-(2.8)). Die Abnahme der Bandlücke $E_{G}$, die in Abb. 2.7 dargestellt ist, führt zu einem zusätzlichen Term in der Diffusionsgleichung [Dri87], welcher der Diffusion entgegenwirkt:

$$
\frac{\partial n}{\partial t}=G+R+\vec{\nabla} D(n) \vec{\nabla} n+\vec{\nabla}\left[n \beta\left(\frac{\mathcal{F}_{1 / 2}\left(\beta \psi_{e}\right)}{\mathcal{F}_{-1 / 2}\left(\beta \psi_{e}\right)}+\frac{\mathcal{F}_{1 / 2}\left(\beta \psi_{h}\right)}{\mathcal{F}_{-1 / 2}\left(\beta \psi_{h}\right)}\right) \frac{\partial E_{g}}{\partial n}\right] \vec{\nabla} n
$$

Den Term der Bandkantenrenormierung und den dichteabhängigen Diffusionskoeffizienten $D(n)$ aus Gl. (5.14) kann man zu einem neuen dichteabhängigen Diffusionskoeffizienten $D^{\prime}(n)$ zusammenfassen:

$$
D^{\prime}(n)=D(n)+n \beta\left(\frac{\mathcal{F}_{1 / 2}\left(\beta \psi_{e}\right)}{\mathcal{F}_{-1 / 2}\left(\beta \psi_{e}\right)}+\frac{\mathcal{F}_{1 / 2}\left(\beta \psi_{h}\right)}{\mathcal{F}_{-1 / 2}\left(\beta \psi_{h}\right)}\right) \frac{\partial E_{g}}{\partial n}
$$

Da $\partial E_{G} / \partial n<0$ ist, führt die Bandkantenrenormierung zu einer Verringerung des Diffusionskoeffizienten. Eine numerische Berechnung von Gl. (7.2) ergibt, daß die Berücksichtigung der Bandkantenrenormierung $\left(\partial E_{G} / \partial n<0\right)$ für Dichten $n<10^{19} \mathrm{~cm}^{-3}$ zu einer Abschwächung der Dichteabhängigkeit des ursprünglichen Diffusionskoeffizienten $D(n)$ und für Dichten $n>10^{19} \mathrm{~cm}^{-3}$ sogar zu einem negativen Diffusionskoeffizienten $D^{\prime}(n)$ führen würde. Ein negativer Diffusionskoeffizient $D$ würde einen Teilchenstrom verursachen, der den Dichtegradienten $\vec{\nabla} n$ nicht reduziert sondern erhöht und würde somit zur Ausbildung einer neuen thermodynamischen Phase, nämlich der der Elektron-Loch-Tröpfchen führen. Die Existenz einer solchen Phase in direkten binären Halbleitern ist zwar vorhergesagt worden [Zim87, konnte aber experimentell nicht bestätigt werden [Hil78, Nak92, Kli95].

Auch die in dieser Arbeit vorgestellten Experimente, in welchen eine schnelle Expansion des Elektron-Loch-Plasmas gefunden wurde, können nicht mit dem Diffusionskoeffizienten aus Gl. (7.2) beschrieben werden. Diese Diskrepanz zwischen der einfachen hydrodynamischen Theorie, die der Gl. (7.2) zugrundeliegt, und dem Experiment könnte aus 
der Tatsache resultieren, daß die Diffusivität von Ladungsträgern mit Wellenvektoren $k \approx k_{f}\left(k_{f} \ldots\right.$ Fermiwellenvektor $)$ und nicht mit $k \approx 0$ beeinflußt wird. Eine detailliertere Vielteilchentheorie [Bla91] zeigt, daß sich die Energiereduzierung im k-Raum nur über ein bis zwei Fermiwellenvektoren $k_{f}$ erstreckt. Deshalb wird der Einfluß der Renormierung der Eigenenergien unter der Annahme von starren Bändern in Gl. (7.2) überschätzt.

Somit kann man feststellen, daß die Renormierung der Eigenenergien aufgrund von Vielteilcheneffekten die Dichteabhängigkeit des Diffusionskoeffizienten von Gl. (5.14) abschwächen würde, jedoch eine Berücksichtigung dieses Effektes eine aufwendige Vielteilchentheorie erfordern würde. Eine einfache Berücksichtigung des Effektes unter der Annahme von starren Bändern führt nicht zu sinnvollen Ergebnissen.

\section{Abschirmung von Ladungsträger Streumechanismen}

Die Abschirmung der Coulombwechselwirkung in einem Hochdichte-Plasma führt nicht nur zu einer Renormierung der Eigenenergien, sondern beeinflußt auch die Streumechanismen von Ladungsträgern, die durch die Coulombwechselwirkung vermittelt werden [Hau93, Zim87].

Für die in dieser Arbeit untersuchte Plasmaexpansion sind insbesondere die Abschirmung der langreichweitigen Fröhlich-Wechselwirkung zwischen Ladungsträgern und LO-Phononen und die Abschirmung der Ladungsträger-Streuung an ionisierten Störstellen zu beachten. Dieses sind die dominierenden Streuprozesse, die den NiedrigdichteDiffusionskoeffizienten $D^{0}$ bei hohen (LO-Phonon-Streuung) bzw. tiefen (ionisierte Störstellen-Streuung) Ladungsträgertemperaturen $T_{c}$ begrenzen (siehe Abb. 6.4).

Die Abschirmung der Ladungsträger LO-Phonon-Wechselwirkung durch ein ElektronLoch-Plasma wurde im Zusammenhang mit der in Kap. 2.2 diskutierten Abkühlung heißer Ladungsträger intensiv untersucht [Yof81, Kas85, Sar88, Col89, Sha99]. Die Abschirmung führt zu einer Verringerung der Streuwahrscheinlichkeit und damit zu einer Erhöhung der Ladungsträgerbeweglichkeiten bei hohen Ladungsträgertemperaturen $T_{c}$. Eine Berücksichtigung der Abschirmung in dem in Kap. 5.2.1 vorgestellten hydrodynamischen Diffusionsmodell ist in erster Näherung mit der Einführung von dichteabhängigen Ladungsträgerbeweglichkeiten $\mu_{c}=\mu_{c}(n)$ möglich. Simulationen mit $\mu_{c}=\mu_{c}(n)$, bei denen die Streuzeiten von Referenz [Sar88] eingesetzt wurden, zeigen, daß die Abschirmung der Ladungsträger-LO-Phonon-Wechselwirkung zu einer weiteren Erhöhung der ambipolaren Diffusivität bei $T_{c}=300 \mathrm{~K}$ führt. Der Beitrag zur Dichteabhängigkeit des Diffusionskoeffizienten $D(n)$ ist jedoch klein, verglichen mit dem des Fermidruckes. Ein weiteres Indiz dafür, daß die Abschirmung der LadungsträgerLO-Phonon-Streuung nicht der dominierende Beitrag für die untersuchte schnelle und dichteabhängige Elektron-Loch-Plasma-Expansion ist, ist die Tatsache, daß der Einfluß dieses Effektes mit abnehmender Ladungsträgertemperatur $T_{c}$ abnehmen sollte und somit die gefundene Temperaturabhängigkeit der Plasmaexpansion insbesondere die stärkere 
Dichteabhängigkeit bei tiefen Temperaturen nicht erklären kann.

Bei tiefen Ladungsträgertemperaturen $T_{c}$ ist die ionisierte Störstellenstreuung der dominierende Streuprozeß. Für eine detaillierte Beschreibung der Plasmaexpansion wäre eine Berücksichtigung auch der Abschirmung dieses Streuprozesses notwendig. Die wesentlichen Aussagen über die Plasmaexpansion bei tiefen Temperaturen $T_{L}$ werden durch eine Berücksichtigung dieses Prozesses jedoch nicht beeinflußt. Eine Berücksichtigung dieses Prozesses mit dichteabhängigen Beweglichkeiten $\mu_{c}=\mu_{c}(n)$ würde nur zu einer Verstärkung der Dichteabhängigkeit von $D=D(n)$ bei tiefen $T_{c}$ führen, jedoch nicht die Abnahme des Niedrigdichte-Diffusionskoeffizienten $D^{0}\left(T_{c}\right)$ (siehe Abb. 6.6) mit abnehmender Temperatur $T_{c}$ für $T_{c}<100 \mathrm{~K}$ beeinflussen. Die Abnahme von $D^{0}\left(T_{c}\right)$ wurde in Kap. 6 als eine der Ursachen für die starke Dichteabhängigkeit der Plasmaexpansion bei tiefen Temperaturen $T_{L}$ diskutiert.

\section{Einfluß der Intervalley-Streuung auf die Plasmaexpansion}

Wie in Kap. 2.2 dargelegt, ist bei einer optischen Anregung von Elektronen mit kinetischen Energien $E_{k i n}$, die größer sind als der energetische Abstand des Leitungsbandminimums zu den Nebenminima an den $L$ - bzw. $X$-Punkten $\Delta_{\Gamma-L}$ bzw. $\Delta_{\Gamma-X}$, eine Streuung der Elektronen in die Seitentäler (Intervalley-Streuung) $X, L$ möglich. Elektronen, die sich in den Seitentälern $X, L$ befinden, besitzen aufgrund ihrer im Vergleich zu den Elektronen im $\Gamma$-Tal größeren effektiven Masse $m^{*}$ eine geringere Beweglichkeit $\mu$ (siehe z.B. [Nus87, Kal96]). Somit würde eine signifikante Besetzung der Zustände in den Seitentälern zu einer Verringerung des Diffusionskoeffizienten (siehe Gl. (5.14) und (5.11)) führen.

Für die Streuung der Elektronen aus dem $\Gamma$-Tal in die Seitentäler wurden in GaAs bei $T_{L}=2 \mathrm{~K}$ Streuzeiten von $\tau_{\Gamma L}=540 \pm 120 \mathrm{fs}$ für Elektronen mit $E_{k i n}=480 \mathrm{meV}$ und $\tau_{\Gamma X}=180 \pm 40 \mathrm{fs}$ für Elektronen mit $E_{k i n}=580 \mathrm{meV}$ gefunden [Ulb89]. Somit ist die Intervalley Streuung ein konkurrierender Prozeß zur Emission von LO-Phononen für die energetische Relaxation von Elektronen. Die Rückstreuung der Elektronen vom $L$-Tal ins $\Gamma$-Tal erfolgt bei $T_{L}=300 \mathrm{~K}$ innerhalb von ca. 2,5 ps [Obe89, Zol89]. Bei der Abkühlung von Raumtemperatur auf tiefe Temperaturen erhöht sich die Streuzeit aufgrund der abnehmenden Zahl der thermischen Phononen um einen Faktor 3 [Zol89]. Die Rückstreuung der Elektronen aus dem $X$-Tal ins $\Gamma$-Tal erfolgt hauptsächlich über den Zwischenschritt der Streuung ins $L$-Tal mit Streuzeiten $\tau_{X L}<1$ ps und anschließender Streuung ins $\Gamma$-Tal [Zol89]. Somit ist für Verzögerungszeiten $\tau>10$ ps keine signifikante Besetzung der Seitentäler $X, L$ zu erwarten.

Experimentell kann diese These z.B. auch anhand der in dieser Arbeit durchgeführten Messungen von $\Delta R_{\text {cis }}(\tau)$ und $\Delta T(\tau)$ mit einer Probephotonenenergie von $E_{\text {probe }} \approx E_{G}+\Delta_{\text {soh }}$ verifiziert werden. Hier nutzt man aus, daß diese $\Delta R$ und $\Delta T$ Messungen nur auf Elektronen im Minimum des $\Gamma$-Tales sensitiv sind (siehe Kap. 3.3). Bei allen in dieser Arbeit verwendeten Pumpphotonenenergien wird das Maximum der $\Delta R_{\text {cis }}(\tau)$ als auch der $\Delta T(\tau)$ Kurven, welches mit der maximalen Anzahl der Elektronen im Minimum des 
$\Gamma$-Tal verknüpft ist, für Zeiten $\tau<10$ ps erreicht (siehe z.B. Inset von Abb. 5.2). Somit bestätigen diese Messungen die Annahme, daß die Rückstreuung der Elektronen aus den Seitentälern in das $\Gamma$-Tal auf einer Zeitskala $\tau<10$ ps stattfindet.

Dieser Fakt zusammen mit der Tatsache, daß der ambipolare Diffusionskoeffizient von der Diffusivität der Löcher (aufgrund ihrer größeren effektiven Masse) dominiert wird, läßt die Schlußfolgerung zu, daß der Einfluß der Intervalley-Streuung auf die untersuchte Plasmaexpansion, insbesondere für Zeiten $\tau>10 p s$, vernachlässigbar ist.

\section{Einfluß des Phononen Windes auf die Plasmaexpansion}

In den in dieser Arbeit durchgeführten Flugzeitmessungen wurden sowohl bei tiefen Gittertemperaturen $T_{L}<10 \mathrm{~K}$ als auch bei Raumtemperatur anfängliche Ausbreitungsgeschwindigkeiten der Plasmen von $v>v_{\text {Schall }}\left(v_{\text {Schall }}^{\text {GaAs }} \approx 5 \times 10^{5} \mathrm{~cm}^{-1}\right)$ gefunden. Aus Tatsache kann man schließen, daß der in Kap. 2.3 vorgestellte Mechanismus des Phononen Windes Ste84] nicht die treibende Kraft der in dieser Arbeit untersuchten Plasmaexpansion in GaAs ist.

\section{Plasmatransport über den Photon-Recycling-Mechanismus}

Wie in Kap. 2.3 dargelegt, wird für den lateralen Transport in Quantenfilmstrukturen ein Plasmatransport über Emission und Reabsorption von Photonen diskutiert [Hil92, Cin90, Fox93, Ble96]. Hierbei wirken die Quantenfilmstrukturen als Wellenleiter für die emittierten Photonen. In Gus97] wird dieser Mechanismus für die Interpretation der im Experiment gefundenen EHP-Expansion mit Überschallgeschwindigkeiten verwendet. Die Tatsache, daß eine Plasmaexpansion mit Geschwindigkeiten $v>v_{\text {Schall }}$ gefunden wurde, läßt jedoch nicht zwingend die Schlußfolgerung zu, daß der Photon-Recycling-Mechanismus die treibende Kraft der Plasmaexpansion ist. Diese hohen Ausbreitungsgeschwindigkeiten können auch unter der Annahme des Fermidruckes als treibender Kraft erklärt werden (siehe Kap. 5.2.1). Eine Zeitabhängigkeit der Expansionsgeschwindigkeiten, welche weitergehende Schlußfolgerungen erlauben würde, konnte aus dem Experiment in [Gus97] nicht bestimmt werden.

Der Transportstrom in dem Photon-Recycling-Mechanismus ist nicht proportional zu dem Dichtegradienten und sollte sich somit nicht mit einer Diffusionsgleichung beschreiben lassen [Fox93]. Im Gegensatz dazu lassen sich die in dieser Arbeit vorgestellten Ergebnisse relativ gut mit dem auf der Diffusionsgleichung basierenden Modell beschreiben. Diese Tatsache führt zu der Schlußfolgerung, daß der Photon-Recycling-Mechanismus anscheinend nicht der dominierende Prozeß für die hier untersuchte Plasmaexpansion senkrecht zur Oberfläche ist. 


\section{Kapitel 8}

\section{Zusammenfassung}

Ziel dieser Arbeit war es, die Ausbreitung von optisch generierten Elektron-Loch-Plasmen in GaAs senkrecht zur Probenoberfläche zu untersuchen, um daraus Rückschlüsse auf die zugrundeliegende treibende Kraft der Plasmaexpansion ziehen zu können. Hierfür wurde im Rahmen dieser Arbeit eine optische Flugzeitmeßmethode entwickelt, welche dann für die Untersuchung der Plasmaexpansion in GaAs bei Raumtemperatur $T_{L}=300 \mathrm{~K}$ und auch bei tiefen Gittertemperaturen $T_{L}<300 \mathrm{~K}$ eingesetzt wurde.

In der Flugzeitmeßmethode wird in einem Anregungs- und Testexperiment die Änderung der Intensität der reflektierten und transmittierten Probepulse $(\Delta R, \Delta T)$ in Abhängigkeit von der Verzögerungszeit $\tau$ einerseits bei Anregung und Detektion auf der selben Probenseite (cis) und andererseits bei Anregung und Detektion auf gegenüberliegenden Seiten der Probe (trans) gemessen. Es wird ausgenutzt, daß in absorbierenden Medien die differentielle Reflexion $\Delta R$ im Gegensatz zur differentiellen Transmission $\Delta T$ nur sensitiv auf Ladungsträger in der Nähe der reflektierenden Oberfläche ist. In dieser Arbeit konnte gezeigt werden, daß der Vergleich von $\Delta R$ in cis und trans Geometrie die Bestimmung der Ankunftszeit des Plasmas $\tau_{H M}$ auf der gegenüberliegenden Probenseite zuläßt. Aus diesen Ankunftszeiten, gemessen bei unterschiedlichen Probendicken $d$, können direkt die zeitabhängigen Expansionsgeschwindigkeiten $v$ der oberflächennah $\left(1 / \alpha_{\text {Pump }}<0,3 \mu \mathrm{m}\right)$ angeregten Ladungsträgerplasmen bestimmt werden.

Die räumliche Auflösung dieser Methode, welche in etwa der halben Absorptionslänge der Probepulse 1/2(1/ $\left.\alpha_{\text {probe }}\right)$ entspricht, konnte in GaAs durch das Austesten des Übergangs vom Spin-Bahn abgespaltenen Lochband (soh) zum Leitungsband (c) auf $\sim 150 \mathrm{~nm}$ erhöht werden. Die zeitliche Auflösung der Methode ist prinzipiell nur durch die Pulsdauer der verwendeten Lichtpulse von $\tau_{\text {puls }} \sim 100$ fs begrenzt.

Diese hier entwickelte Flugzeitmeßmethode ermöglicht zum ersten Mal eine direkte Bestimmung der Zeitabhängigkeit der Expansionsgeschwindigkeit von Elektron-LochPlasmen senkrecht zur Oberfläche mit einer sub- $\mu$ m räumlichen und sub-ps zeitlichen Auflösung.

Nachdem die Messung von $\Delta R_{c i s}(\tau)$ und $\Delta T_{\text {cis }}(\tau)$ erste Hinweise auf eine schnelle 
Elektron-Loch-Plasma-Expansion mit zunehmender Pumpfluenz ergeben hatte, wurde mit Hilfe der Flugzeitmeßmethode die Plasmaexpansion in GaAs bei Raumtemperatur detailliert untersucht. Bei einer Pumpfluenz von $F=800 \mu \mathrm{Jcm}^{-2}$ wurden relativ scharfe Ladungsträgerfronten gefunden, welche sich mit anfänglich hohen Geschwindigkeiten von $v(\tau=20 \mathrm{ps})=14 \times 10^{5} \mathrm{~cm} / \mathrm{s}$ ausbreiten. Die Ausbreitungsgeschwindigkeit sinkt mit zunehmender Verzögerungszeit mit $v \propto \tau^{-0,67}$ auf $v(\tau=400 \mathrm{ps})=2 \times 10^{5} \mathrm{~cm} / \mathrm{s}$. Mit zunehmender Pumpfluenz wurden deutlich höhere Expansionsgeschwindigkeiten gemessen. Bei einer festen Probendicke $d$ kann die Korrelation zwischen der Ankunftszeit des Plasmas $\tau_{H M}$ und der Pumpfluenz $F$ mit: $\tau_{H M} \propto 1 / F^{0,45}$ beschrieben werden.

Die experimentellen Ergebnisse wurden mit numerischen Simulationen im Rahmen eines isothermischen hydrodynamischen Diffusionsmodelles mit einem dichteabhängigen Diffusionskoeffizienten verglichen. Eine qualitativ gute Übereinstimmung der Simulation mit dem Experiment wird unter der Annahme erzielt, daß der Fermidruck, welcher durch das Einsetzen der Entartung bei hohen Dichten verursacht wird, die treibende Kraft der untersuchten Plasmaexpansion ist. Die Ausbildung von relativ steilen Ladungsträgerfronten kann dadurch erklärt werden, daß die schnell diffundierenden Ladungsträger innerhalb des Hochdichte-Plasmas von langsam diffundierenden Ladungsträgern an der Vorderfront des Plasmas zurückgehalten werden [Zie00a].

Im Anschluß an die Raumtemperatur-Untersuchungen, wurden Flugzeitmessungen bei Gittertemperaturen $T_{L}<300 \mathrm{~K}$ durchgeführt. Während bei abnehmender Gittertemperatur von $T_{L}=300 \mathrm{~K}$ auf $T_{L}=4 \mathrm{~K}$ die Absolutwerte der Expansionsgeschwindigkeiten ansteigen, ergibt sich für $T_{L}=300 \mathrm{~K}$ und $T_{L}=7 \mathrm{~K}$ ein vergleichbarer funktionaler Zusammenhang zwischen der Expansionsgeschwindigkeit $v$ und der Verzögerungszeit $\tau$ (7K: $\left.v \propto \tau^{-0,65}\right)$. Bei tiefen Temperaturen $\left(T_{L}=4 \mathrm{~K}\right)$ wird im Vergleich $\mathrm{zu} T_{L}=300 \mathrm{~K}$ eine wesentlich stärkere Abhängigkeit der Expansionsgeschwindigkeit von der Pumpfluenz gefunden. Bei fester Probendicke hängt die Ankunftszeit des Plasmas von der Fluenz wie folgt ab: $\tau_{H M} \propto 1 / F^{1,1}$ [Zie00b].

Für eine theoretische Beschreibung der Plasmaexpansion in GaAs für $T_{L}<300 \mathrm{~K}$ im Rahmen des hydrodynamischen Diffusionsmodelles ist eine explizite Berücksichtigung der Ladungsträgerabkühlung notwendig. Die Ursache dafür sind die im Vergleich zu $T_{L}=300 \mathrm{~K}$ wesentlich längeren Abkühlzeiten der Ladungsträger $(\sim 100 \mathrm{ps})$ bei tiefen Gittertemperaturen. Die gefundene stärkere Dichteabhängigkeit der Expansion bei tiefen Temperaturen kann auf zwei wesentliche Ursachen zurückgeführt werden. Zum einen setzt bei tiefen Ladungsträgertemperaturen die Entartung schon bei geringeren Dichten ein und führt somit zu einer stärkeren Dichteabhängigkeit des Diffusionskoeffizienten $D(n)$, zum anderen verursacht auch die implizite Zeitabhängigkeit des Diffusionskoeffizienten $D\left(n, T_{c}(\tau)\right)$ eine stärkere Dichteabhängigkeit der Expansionsgeschwindigkeiten.

Somit kann die schnelle und dichteabhängige Elektron-Loch-Plasma-Expansion in GaAs, sowohl bei Raumtemperatur, als auch bei tiefen Gittertemperaturen $T_{L}$, in einem hydrodynamischen Diffusionsmodell mit dem Fermidruck als treibender Kraft beschrieben werden. 


\section{Anhang}

\section{Bestimmung des dichteabhängigen Diffusionskoeffizienten}

Ausgangspunkt ist die Teilchenstromdichte $J$, welche von dem Gradienten der Quasiferminiveaus getrieben wird.

$$
J=-\frac{1}{q^{2}} \frac{\sigma_{e} \sigma_{h}}{\sigma_{e}+\sigma_{h}}\left[\nabla\left(\psi_{h}+\psi_{e}\right)\right]
$$

Hierbei ist $\sigma_{c}(\mathrm{c}=\mathrm{e}, \mathrm{h})$ die dichteabhängige elektrische Leitfähigkeit der Elektronen bzw. Löcher:

$$
\sigma_{c}=e n_{c} \mu_{c}^{0} \frac{\mathcal{F}_{0}\left(\beta \psi_{c}\right)}{\mathcal{F}_{1 / 2}\left(\beta \psi_{c}\right)}
$$

mit der Beweglichkeit $\mu_{c}^{0}$ der Ladungsträger im nichtentarteten Grenzfall und den n-ten Fermiintegralen $\mathcal{F}_{n}(z)$, die definiert sind als:

$$
\mathcal{F}_{n}(z)=\frac{1}{\Gamma(n+1)} \int_{0}^{\infty} \frac{x^{n}}{\exp (x-z)+1} d x .
$$

Die Gradienten der Quasiferminiveaus von Gleichung 1 sollen nun in Dichtegradienten umgerechnet werden. Die lokale Ladungsträgerdichte $n_{c}$ ergibt sich aus der Integration der Fermiverteilungsfunktion $f_{c}(\mathbf{r}, \mathbf{p})$ über dem k-Raum.

$$
n_{c}=2\left[\frac{m_{c} \beta}{2 \pi \hbar^{2}}\right]^{3 / 2} \mathcal{F}_{1 / 2}\left(\beta \psi_{c}\right),
$$


Für den Dichtegradienten $\nabla n_{c}$ ergibt sich aus Gleichung 4:

$$
\begin{aligned}
\nabla n_{c} & =\nabla\left(2\left[\frac{m_{c} \beta}{2 \pi \hbar^{2}}\right]^{3 / 2} \mathcal{F}_{1 / 2}\left(\beta \psi_{c}\right)\right) \\
& =2\left[\frac{m_{c} \beta}{2 \pi \hbar^{2}}\right]^{3 / 2} \frac{\partial}{\partial\left(\beta \psi_{c}\right)}\left[\mathcal{F}_{1 / 2}\left(\beta \psi_{c}\right)\right] \beta \nabla \psi_{c} \\
& =2\left[\frac{m_{c} \beta}{2 \pi \hbar^{2}}\right]^{3 / 2} \mathcal{F}_{-1 / 2}\left(\beta \psi_{c}\right) \beta \nabla \psi_{c}
\end{aligned}
$$

wobei man die Differentiationsregel für die Fermiintegrale ausgenutzt hat:

$$
\frac{d}{d z} \mathcal{F}_{n}(z)=\mathcal{F}_{n-1}(z)
$$

Somit ergibt sich für den Gradienten des Quasiferminiveaus $\nabla \psi_{c}$ :

$$
\begin{aligned}
\nabla \psi_{c} & =\frac{\left(2 \pi \hbar^{2}\right)^{3 / 2}}{\left(2 m_{c} \beta\right)^{2 / 3} \beta \mathcal{F}_{-1 / 2}\left(\beta \psi_{c}\right)} \\
& =\frac{\mathcal{F}_{1 / 2}\left(\beta \psi_{c}\right)}{n_{c} \beta \mathcal{F}_{-1 / 2}\left(\beta \psi_{c}\right)} \nabla n_{c}
\end{aligned}
$$

Setzt man nun Gleichung 8 in Gleichung 1 ein, so erhält man:

$$
J=-\frac{1}{q^{2}} \frac{1}{n \beta} \frac{\sigma_{e} \sigma_{h}}{\sigma_{e}+\sigma_{h}}\left[\frac{\mathcal{F}_{1 / 2}\left(\beta \psi_{h}\right)}{\mathcal{F}_{-1 / 2}\left(\beta \psi_{h}\right)}+\frac{\mathcal{F}_{1 / 2}\left(\beta \psi_{e}\right)}{\mathcal{F}_{-1 / 2}\left(\beta \psi_{e}\right)}\right] \nabla n
$$

Aus dem Vergleich mit der Diffusionsgleichung: $J=-D \nabla n$ erhält man nun für den dichteabhängigen Diffusionskoeffizienten $D(n)$ :

$$
D(n)=\frac{1}{q^{2}} \frac{1}{n \beta} \frac{\sigma_{e} \sigma_{h}}{\sigma_{e}+\sigma_{h}}\left[\frac{\mathcal{F}_{1 / 2}\left(\beta \psi_{h}\right)}{\mathcal{F}_{-1 / 2}\left(\beta \psi_{h}\right)}+\frac{\mathcal{F}_{1 / 2}\left(\beta \psi_{e}\right)}{\mathcal{F}_{-1 / 2}\left(\beta \psi_{e}\right)}\right]
$$




\title{
Verzeichnis häufig verwendeter Abkürzungen und Symbole
}

\author{
c Leitungsband (engl. conduction band) \\ $c=e, h \quad$ Ladungsträger: (e) Elektronen, (h) Löcher \\ cis Anregungsgeometrie bei der Pump- und Probepulse auf derselben \\ Probenseite eintreffen \\ D Diffusionskoeffizient \\ d Probendicke \\ $E=\hbar \omega \quad$ Photonenenergie \\ e Elektronen \\ e Elementarladung \\ $E_{G} \quad$ Bandlückenenegie \\ EHL Elektron-Loch-Flüssigkeit (engl. electron hole liquid) \\ EHP Elektron-Loch-Plasma (engl. electron-hole plasma) \\ $F \quad$ Fluenz der Pumppulse \\ $\mathcal{F}_{n} \quad$ n-tes Fermiintegral \\ $f_{e}, f_{h} \quad$ Besetzungfunktion der Elektronen (e) und Löcher (h) \\ FWHM volle Halbwertsbreite \\ Gl. Gleichung \\ h Löcher (engl. hole) \\ hh/lh/soh Löcher des Schwer-/ Leicht-/ und des Spin-Bahn-abgespaltenen Lochbandes \\ $J \quad$ Teilchenstromdichte \\ j Stromdichte \\ $\vec{k} \quad$ Wellenvektor der Elektronen bzw. Löcher \\ LO/TO longitudinal optisch / transversal optisch \\ $m_{c} \quad$ effektive Masse der Elektronen $(\mathrm{c}=\mathrm{e})$ bzw. Löcher $(\mathrm{c}=\mathrm{h})$ \\ n Teilchendichte \\ $n \quad$ Brechnungsindex \\ OPA Optisch Parametrischer Verstärker \\ $R \quad$ Reflexionskoeffizient \\ Reg A Regenerativer Verstärker (Coherent Model 9095) \\ $T \quad$ Transmissionskoeffizient \\ $T_{c} \quad$ Ladungsträgertemperatur
}


$T_{L} \quad$ Gittertemperatur

trans Anregungsgeometrie bei der Pump- und Probepulse auf gegenüberliegenden Probenseiten eintreffen

$v \quad$ Geschwindigkeit

z Ortskoordinate senkrecht zur Oberfläche

$\alpha \quad$ Absorptionskoeffizient

$\Delta R \quad$ differentielle Reflexion

$\Delta T \quad$ differentielle Transmission

$\kappa \quad$ Extinktionskoeffizient

$\mu \quad$ Beweglichkeit

$\Psi \quad$ Quasiferminiveau

$\sigma \quad$ Leitfähigkeit

$\tau \quad$ Verzögerungszeit

$\tau_{H M} \quad$ Ankunftszeit des Plasmas

$\tau_{\text {puls }} \quad$ Pulslänge 


\section{Literaturverzeichnis}

[Ale95] A. Alexandrou, V. Berger, D. Hulin, Phys. Rev. B 52, 4654 (1995).

[Asp83] D.E. Aspnes, A.A. Studna, Phys. Rev. B 27,985 (1993).

[Bec88] P.C. Becker, H.L. Fragnito, C.H. Brito Cruz, R.L. Fork, J.E. Cunningham, J.E. Henry, C.V. Shank, Phys. Rev. Lett. 61, 1647 (1988).

[Bla82] J.S. Blakemore, Semiconductor and other major properties of GaAs, J. Appl. Phys. 53, R123-R181 (1982).

[Bla91] R. Blank, H. Haug Phys. Rev. B 44, 10513 (1991).

[Ble96] I.J. Blewett, N.R. Gallaher, A.K. Kar, B.S. Wherrett, J. Opt. Soc. Am. B 13, 779 (1996).

[Böh92] G. Böhne, Dissertation, Universität Göttingen, (1992).

[Bro87] S.D. Brorson, J.G. Fujimoto, E.P. Ippen, Phys. Rev. Lett. 59, 1962 (1987).

[Che87] J.R. Chelikowski, M.L. Cohen, Phys. Rev. B, 14, 556 (1976).

[Cin90] R. Cingolani, K. Ploog, A. Cingolani, C. Moro, M. Ferrara, Phys. Rev. B 42, 2893 (1990) und R. Cingolani, K. Ploog, A. Cingolani, C. Moro, M. Ferrara, Phys. Rev. B 45, 8785 (1992).

[Coc97] J.W. Cockburn, J.J. Finley, M.S. Skolnick, P. Wisniewski, R. Grey, G. Hill, M.A. Pate, Appl. Phys. Lett. 70, 622 (1997).

[Col89] J.H. Collet, Phys. Rev. B 39, 7659, (1989).

[Col83] C.L. Collins, P.Y. Yu, Phys. Rev. B 27,2602 (1983).

[Die96] J.C. Diels, W. Rudolph, Ultrashort Laser Pulse Phenomena, Academic Press (1996).

[Dre93] S. Dreher, Diplomarbeit, Universität Göttingen (1993).

[Dri87] H.M. van Driel, Phys. Rev. B 35, 8166 (1987). 
[Ell57] R.J. Elliot, Phys. Rev. 108, 1384 (1957).

[Ent00] V. Entiliani, T. Guenther, C. Lienau, R. Notzel, K.H. Ploog, Phys. Rev. B 61, R10583 (2000).

[Fas90] G. Fasol, W. Hackenberg, H.P. Hughes, K. Ploog, E. Bauser, H. Kano, Phys. Rev. B 41, 1461 (1990).

[Feh82] G.W. Fehrenbach, W. Schäfer, J. Treusch, R.G. Ulbrich, Phys. Rev. Lett. 49 1281 (1982).

[For84] R.L. Fork, O.E. Martinez, J.P. Gordon, Optics Lett., 9, 150 (1984)

[Fox93] E.C. Fox H.M. van Driel, Phys. Rev. B 47, 1663 (1993).

[Fre94] S. Freund Dissertation, Universität Göttingen (1994).

[Frö85] D. Fröhlich, A. Nothe, K. Reimann, Phys. Rev. Lett. 55, 1335 (1985).

[Gie98] H. Gießen, A. Knorr, S. Haas, S.W. Koch, S. Linden, J, Kuhl, M. Hetterich, M. Grün, C. Klingshirn, Phys. Rev. Lett. 814260 (1998).

[Gre95] M. J. Gregor, Dissertation Göttingen, Cuvillier Verlag (1995).

[Gro98] S. Grosse, Dissertation LMU München, Cuvillier Verlag (1998).

[Gus97] W. Gao, V. Gusev, C. Glorieux, J. Thoen, G. Borghs, Opt. Commun. 143, 19 (1997).

[Hau93] H. Haug and S. Koch, Quantum Theory of optical and Electronic Properties of Semiconductors, World Scientific, Singapore, 2nd ed.(1993).

[Hen77] J.C. Hensel, T.G. Phillips, G.A. Thomas, Solid State Phys. 32, 88 (1977).

[Het00] J. Hetzler, A. Brunner, T. Schimmel, M. Wegener, S. Leu, S. Nau, W. Stolz, phys. stat. sol. (b) 221, 425 (2000).

[Hil78] O. Hildebrand, E.O. Goebel, K.M. Romanek, H. Weber, G. Mahler, Phys. Rev. B 17, 4775 (1978).

[Hil86] H. Hillmer, G. Mayer, A. Forchel, K.S. Löchner, E. Bauser, Appl. Phys. Lett. 49, 948 (1986).

[Hil91] H. Hillmer, A. Forchel, T. Kuhn, G. Mahler, H.P. Maier, Phys. Rev. B 43 13992 (1991).

[Hil92] H. Hillmer, S. Hansmann, A. Forchel, Phys. Rev. B 45, 8782 (1992) und Referenzen darin. 
[Hil70] D.E. Hill, J. Appl. Phys. 41, 1815 (1970).

[Jac89] C. Jacoboni, P. Lugli, The Monte Carlo Method for Semiconductor Device Simulation, Springer Verlag, Wien (1989)

[Jef83] Electron-Hole Droplets in Semiconductors ed. C.D. Jeffries, L.V. Keldysh, Modern Problems of Condensed Matter Science Vol. 6, North Holland, Amsterdam (1983).

[Jos90] R.P. Joshi, K.T. Tsen, D.K. Ferry, Phys Rev. B 41, 9899 (1990).

[Kal96] H. Kalt, Optical Propeties of III-V Semiconductors: The Influence of MultiValley Band Structures, Springer Series in Solid state Sciences 120 1st. ed., Berlin, (1996).

[Kas85] J.A. Kash, J.C. Tsang, J.M. Hvam, Phys. Rev. Lett. 54, 2151 (1985).

[Kel68] L.V. Keldysh, in Proc. 9th. Int. Conf. Phys. Semicond. (ICPS) Moscow, p. 1303 (1968).

[Kli95] C.F. Klingshirn Semiconductor Optics, Springer, Berlin (1995).

[Koc00] Proceedings of the Sixth International Workshop on Nonlinear Optics and Excitation Kinetics in Semiconductors (NOEKS) ed. S.W. Koch in phys. stat. sol. (b) 221 (2000).

[Kor83] D.V. Korbutyak, V.G. Litovchenko, phys. stat. sol.(b), 12087 (1983).

[Kre81] H.J. Kreuzer, Nonequlibrium Thermodynamics and its Statistical Foundations, Clarendon, Oxford (1981).

[Kur64] N.A. Kurnit, I.A. Abella, S.R. Hartmann, Phys. Rev. Lett. 13, 567 (1964).

[Lan87] Landoldt-Börnstein, Zahlenwerte und Funktionen aus Naturwissenschaft und Technik, Neue Serie Bd. III/22a, Springer, Berlin (1987).

[Lan96] P. Langot, R. Tommasi, F. Vallee, Phys. Rev. B 54, 1775 (1996).

[Leh79] R.F. Leheny, J. Shah, R.L. Fork, C.V. Shank, A. Migus, Solid State Commun. 31, 809 (1979).

[Lin79] D. van der Linde, R. Lambrich, Phys. Rev. Lett. 42, 1090 (1979).

[Lug87] P. Lugli, C. Jacoboni, L. Reggiani, P. Kocevar, Appl. Phys. Lett. 50, 1251 (1987).

[Mad96] O. Madelung, Introduction to Solid State Theory, Springer Series in Solid State Science 3, 3nd ed., Berlin (1996). 
[Mad64] O. Madelung, Physics of III-V Compounds, Wiley, New York (1964).

[Mah88] G. Mahler T. Kuhn, A. Forchel, H. Hillmer: in Optical nonlinearities and instabilities in semiconductors, ed. H. Haug, Academic Press, Boston (1988).

[Mea71] A.L. Mears, R.L. Stradling, J. Phys. C 4, L22 (1971).

[Mot74] N.F. Mott Metall-Insulator Transitions, Taylor, Francis, London (1974).

[Mys86] A. Mysyrowitz, D. Hulin, A. Antonetti, A. Migus, W.T. Masselink, H. Morkoc, Phys. Rev. Lett. 56, 2748 (1986).

[Nak92] H. Nakata, A. Uddin, E. Otsuka, Semicond. Sci. Technol.7, 1266 (1992).

[Neh96] J. Nehls, T. Schmidt, U. Merkt, D. Heitman, A.G. Norman, R.A. Stradling, Phys. Rev. B 54, 7651 (1996).

[Nus87] M.C. Nuss, D.H. Auston, F. Capasso, Phys. Rev. Lett. 58, 2355 (1987).

[Obe89] D.Y. Oberli, J. Sha, T.C. Damen, Phys. Rev. B 40, 1323 (1989).

[Ou85] J.L. Oudar, D. Hulin, A. Migus, A.N. Antonetti, F. Alexandre, Phys. Rev. Lett. 55, 2074 (1985).

[Pan69] M: B: Panisch, H.C. Casey, J. Appl. Phys. 40, 163 (1969).

[Pet92] M. Petrauskas, S. Juodkazis, V. Netikis, M. Willander, A. Ouacha, B. Hammerlund, Semicond. Sci. Technol.7, 1355 (1992).

[Pey93] N. Peyghambarian, S.W. Koch, A. Mysyrowicz, Introduction to Semiconductor Optics, Prentice-Hall Inc., New Jersey (1993).

[Pil85] Proceedings of the Third Trieste ICTP-IUPAP Semiconductor Symposium ed. M.H. Pilkuhn in J. of Lumin. 30, North Holland, Amsterdam (1985).

[Pol96] E. Poles, S.Y. Goldberg, B. Fainberg, D.Huppert, M.C. Hanna, Y. Rosenwaks, J. Appl. Phys. 80, 5129 (1996).

[Pol99] E. Poles, D.Huppert, M.C. Hanna, Y. Rosenwaks, J. Appl. Phys. 86, 3481 (1999)

[Poe87] W. Pötz, Phys. Rev. B 36, 5016 (1987).

[Pug81] M. Pugnet, J. Collet, A. Cornet, Solid State Commun. 38,531 (1981).

[Ree94] M.K. Reed, M.K. Steiner-Shephard, D.K. Negus, Optics Lett., 19, 1855 (1994).

[Ree96] M.K. Reed, M.K. Steiner-Shephard, IEEE J. of Quantum Electronics, 32, 1273 (1996). 
[Ree95] M.K. Reed et al. Optics Lett., 20, 605 (1995) and M.K. Reed et al. J. Opt. Soc. Am. B, 12, 2229 (1995).

[Ric77] T.M. Rice, Solid State Phys. 32, 1 (1977).

[Rom81] K.M. Romanek, H. Nather, J. Fischer, J. Lumin., 24, 585 (1981).

[Sar88] S. Das Sarma, J.K. Jain, R. Jalabert, Sol. State Elec. 31, 695 (1988).

[Sän96] K. Sändig, Diplomarbeit, Universität Göttingen (1996).

[Sch99] A. Schülzgen, R. Binder, M.E. Donovan, M. Lindberg, K. Wundke, H.M. Gibbs, G. Khitrova, N. Peygambarian, Phys. Rev. Lett. 822346 (1999).

[See92] K. Seeger Semiconductor physics, Springer, Wien, (1973).

[Sha99] J. Shah Ultrafast Spectroscopy of Semiconductors and Semiconductor Nanostructures, Springer Series in Solid state Sciences 115 2nd ed., Berlin, (1999).

[Sha92] Hot Carriers in Semiconductor Nanostructures: Physics and Applications ed. by J. Shah, Academic Press, San Diego (1992).

[Sha78] C.V. Shank, D.H. Auston, E.P. Ippen, O. Teschke, Sol. State Comm. 26, 567 (1978).

[Sho49] W. Shockley, Bell System Tech. J. 28, 435 (1949).

[Shu87] M. Shur, GaAs Devices and Circuits, Plenum Press, New York (1987).

[Smi89] L.M. Smith, J.S. Preston, J.P. Wolfe, D.R. Wake, J. Klem, T. Henderson, H. Markoc, Phys. Rev. B 39, 1862 (1989).

[Sti70] G.E. Stillman, C.M. Wolfe, J.O. Dimmock, J. Phys. Chem. Solids 31,1199 (1970) zitiert nach [Bla82].

[Ste84] F. M. Sterenka and J.P. Wolfe, Phys. Rev. Lett. 53, 2181 (1984).

[Str93] U. Strauss, W.W. Rühle, Appl. Phys. Lett. 62, 55 (1993).

[Tan80] S. Tanaka, H. Kobayashi, H. Sato, H. Shionoya, J. Phys. Soc. Jpn. 49, 1051 (1980).

[Tri88] M. Trinn, Diplomarbeit, Universität Dortmund (1988).

[Tse89] K.T. Tsen, O.F. Sankey, G. Halama, S.-C.Y. Tsen, H. Marcoc, Phys. Rev. B 39, 6276 (1989) and K.T. Tsen, G. Halama, O.F. Sankey, S.-C.Y. Tsen, H. Marcoc, Phys. Rev. B 40, 8103 (1989). 
[Ulb73] R.G. Ulbrich, Phys. Rev. B 85719 (1973).

[Ulb89] R.G. Ulbrich, J.A. Kash, J.C. Tsang, Phys. Rev. Lett. 62, 949 (1989).

[Vas82] P. Vashishta, R.K. Kalia, Phys. Rev. B 25, 6492 (1982).

[Vol99] M. Vollmer, H. Giessen ,W. Stolz, L. Ghislain, V. Elings, Appl. Phys. Lett. 74, 1791 (1999).

[Wei88] A.M. Weiner, J.P. Heritage, E.M. Kirschner, J.Opt.Soc.Am. B, 5, 1563 (1988).

[Wit99] T. Witte, Diplomarbeit, Universität Göttingen (1999).

[Woo72] F. Wooten, Optical Properties of Solids, Academic Press, New York (1972).

[Yof81] E.J. Yoffa, Phys. Rev. B 23, 1909 (1981).

[Yoo92] H.W. Yoon, D.R. Wake, J.P. Wolfe, H. Markoc, Phys. Rev B 46, 13461 (1992).

[Zhu96] X. Zhu, M.S. Hybertsen, P.B. Littlewood, Phys. Rev. B 54, 13575 (1996).

[Zie00a] R. Ziebold, T. Witte, M. Hübner, R.G. Ulbrich, Phys. Rev. B 6116610 (2000).

[Zie00b] R. Ziebold, T. Witte, R.G. Ulbrich, phys. stat. sol. (b) 221435 (2000).

[Zim87] R. Zimmermann, Many Particle Theory of Highly Excited Semiconductors, Teubner Texte für Physik 18, Teubner Verlag, Leipzig (1987).

[Zol89] S. Zollner, S. Gopalan, M. Cardona, Appl. Phys. Lett. 54614 (1989), and S. Zollner, S. Gopalan, M. Cardona, J. Appl. Phys. 681682 (1990).

[Zsc73] K.H. Zschauer, in GaAs and Related Compounds,Inst. Phys. Conf. Ser. 17, 3 (1973). 


\section{Danksagung}

An dieser Stelle möchte ich mich bei allen bedanken, die durch ihre Unterstützung zum Gelingen dieser Arbeit beigetragen haben.

Mein besonderer Dank gilt Herrn Prof. R.G. Ulbrich, der diese Arbeit betreut hat und für Diskussionen immer offen war.

Herrn Prof. W. Felsch danke ich für die Übernahme des Zweitgutachtens.

Allen Mitarbeitern des 4. Physikalischen Institutes möchte ich für die angenehme Arbeitsatmosphäre am Institut danken. Insbesondere die Diskussionen über alle anstehenden größeren und kleineren Probleme in der von Peter Blome und Martin Wenderoth wiederbelebten Kaffeerunde haben sehr zum Erfolg dieser Arbeit beigetragen.

Bei Thomas Witte möchte ich mich für die gute Zusammenarbeit bei der Durchführung der Experimente bedanken.

Besonders möchte ich mich bei Joel Nägerl für die sehr gute Zusammenarbeit im Femto-Lab bedanken. Ohne die gegenseitige Unterstützung bei allen organisatorischen, technischen und physikalischen Problemen wäre diese Arbeit kaum möglich gewesen.

Martin Wenderoth möchte ich für die motivierenden und aufmunternden Diskussionen danken, die sehr zum Gelingen der Arbeit beigetragen haben.

Für die Präparation der in den Experimenten verwendeten Proben danke ich Christoph Nacke und Carsten Sändig.

Zu technischen Problemen wurden aufgrund der guten Kooperation mit den Werkstätten schnell Lösungen gefunden. Mein Dank gilt besonders Bernhard Spicher, sowie Erwin Sonnenberg und Uwe Frentzel als Vertreter der Feinmechanik-Werkstatt und Klaus Langohr als Vertreter der Elektronik-Werkstatt.

Auf die volle Unterstützung von Dr. H.-D. Schulte, Edith Rohrmoser und Gerda Brocks in organisatorischen sowie finanziellen Angelegenheiten konnte ich mich stets verlassen, auch dafür vielen Dank.

Für das Korrekturlesen dieser Arbeit möchte ich mich bei Joel Nägerl, Dr. Martin Wenderoth, Tobias Kampfrath, meinem Bruder Falk und Hannelore Heins bedanken.

Bei allen Freunden und besonders auch bei meinen Brüdern und Eltern bedanke ich mich für viele schöne Stunden und für die fortwährende seelische und moralische Unterstützung. Besonders möchte ich mich an dieser Stelle bei meiner Freundin Susanne Heins bedanken, die mir den Rückhalt und die Kraft für diese Arbeit gegeben hat. 



\section{Lebenslauf}

\section{Ralf Ziebold}

geboren am 10.2.1971 in Neustrelitz (Mecklenburg)

09/77-08/79 POS (Grund- und Realschule) „Hans Beimler“, Neustrelitz

09/79-08/87 POS „Wladimir Komarow“, Neustrelitz

09/87-08/89 EOS (Gymnasium) „Clara Zetkin“, Neustrelitz

$08 / 89$

Allgemeine Hochschulreife

09/89-08/90 Praktikum im Elektro-Anlagenbau-Neustrelitz

09/90-08/91 Studium der Elektrotechnik/Elektronik an der Technischen Hochschule Wismar

09/91-08/93 Grundstudium der Physik an der Universität Rostock

09/93-02/97 Hauptstudium der Physik an der Georg-August-Universität Göttingen

03/97 Diplom in Physik an der Universität Göttingen Thema der Diplomarbeit: „Zeitaufgelöste Strahlablenkungsspektroskopie an Galliumarsenid“

seit 02/97 Wissenschaftlicher Mitarbeiter am IV. Physikalischen Institut der Universität Göttingen mit Gelegenheit zur Promotion 